Japan. J. Math.

Vol. 7, No. 2, 1981

\title{
Calcul des variations analytiques
}

\author{
Par Hiroshi Yamaguchi \\ (Communiqué par Prof. Y. Kusunoki, le 13, Avril, 1981)
}

\section{§Introduction}

Soit $D$ un ouvert connexe multivalent en nombre fini, $p$, étale au-dessus du plan d'une variable complexe $w$ tel que la frontière de $D$, qui s'écrit $C$, consiste en nombre fini, $q$, de courbes fermées et $D$ puisse contenir un nombre fini de points de ramification, soient $\xi_{1}, \cdots, \xi_{m}$. Nous allons déformer analytiquement un peu l'ouvert $D$ avec paramètre complexe $z$ comme suit: Quand $z$ $\left(=\left(z_{1}, \cdots, z_{n}\right)\right)$ se varie dans un polydisque $\Delta\left(=\Delta_{1} \times \cdots \times \Delta_{n}\right)$ où $\Delta_{i}=\left\{\left|z_{i}\right|<\rho_{i}\right\}$ dans l'espace de $n$ variables complexes $z_{1}, \cdots, z_{n}$, on fait se varier chaque point de ramification $\xi_{i}$ et chaque point frontière $\zeta$, de $D$ de façon que $\xi_{i}(z)$ (resp. $\xi(z, \zeta))$ soit fonction analytique au sens complexe par rapport à $z$ dans $\Delta$ telle que $\xi_{i}(0)=\xi_{i}$ (resp. $\left.\xi(0, \zeta)=\zeta\right)$ et $\xi(z, \zeta) \neq \xi\left(z, \zeta^{\prime}\right)$ pour $\zeta \neq \zeta^{\prime}$. En posant $C(z)=\{\xi(z, \zeta): \zeta \in C\}$, on a pour tout $z \in \Delta$ l'ouvert connexe $D(z)$ multivalent en $p$ étalé au-dessus du plan $w$ dont la frontière est $C(z)$ et les points de ramification sont $\left\{\xi_{1}(z), \cdots, \xi_{m}(z)\right\}$ tel que $D(0)=D$. Chaque $D(z)$ est topologiquement équivalent à $D$, mais non holomorphiquement en général. La correspondance ainsi obtenue: $z \rightarrow D(z)$ où $z \in \Delta$ est dite variation analytique. Etant données deux variations analytiques $z \rightarrow D_{i}(z)$ où $z \in \Delta(i=1,2)$, elles sont dites équivalentes, quand il existe pour tout $z$ fixé dans $\Delta$ une transformation conforme $\varphi(z, w)$ de $D_{1}(z)$ sur $D_{2}(z)$ telle que $\varphi(z, w)$ soit analytique par rapport à $z$ dans $\Delta$. Il ne me semble pas que telle variation manque d'intérêt. On a montré par exemple une propriété suivante (p. 620 de [17]): soit $z \rightarrow D(z)$ où $z \in \Delta$ une variation analytique telle que $n=1$ et la frontière $C(z)$ consiste en des cercles entiers $C_{i}(z)(i=1, \cdots, q)$ avec centre $a_{i}(z)$ et rayon $r_{i}(z)$ dans le plan $w$. Alors ils s'expriment, à des fonctions holomorphes près, $a_{i}(z)=$ $=\partial u_{i}(z) / \partial z$ et $r_{i}(z)=e^{u_{i}(z)}$ où $u_{i}(z)$ est une fonction à valeur réelle $\Delta$ dans satisfaisant à l'équation différentielle de Liouville $\partial^{2} u / \partial z \partial \bar{z}=e^{2 u}$.

Soit $z \rightarrow D(z)$ où $z \in \Delta$ une variation analytique. Il existe sur chaque $D(z)$ la fonction de Green $g_{a}(z, w)$ avec pôle logarithmique au point $a$ et on note $\lambda(z, a)$ la constante de Robin de $D(z)$ par rapport au même point $a$. Elle se développe dans un voisinage de $a$ 


$$
g_{a}(z, w)=\log \frac{1}{|w-a|}+\lambda(z, a)+\operatorname{Re}\left\{\sum_{n=1}^{\infty} a_{n}(z, a)(w-a)^{n}\right\}
$$

Etant donnée une valeur réelle $f(\zeta)$ ne pas dépendant de $z \in \Delta$ correspondante au point contière $\xi(z, \zeta)$ de $D(z)$, il existe sur $D(z)$ une solution $H_{f}(z, w)$ du problème de Dirichlet par rapport à $f$. On aura des formules de variation symétriques,

$$
\begin{aligned}
& \frac{\partial^{2} g_{a}(z, b)}{\partial z_{i} \partial \bar{z}_{j}}=-\frac{2}{\pi} \iint_{D(z)}\left\{\frac{\partial^{2} g_{a}(z, w)}{\partial z_{i} \partial \bar{w}} \frac{\partial^{2} g_{b}(z, w)}{\partial \bar{z}_{j} \partial w}+\frac{\partial^{2} g_{a}(z, w)}{\partial \bar{z}_{j} \partial w} \frac{\partial^{2} g_{b}(z, w)}{\partial z_{i} \partial \bar{w}}\right\} d u d v ; \\
& \frac{\partial^{2} \lambda(z, a)}{\partial z_{i} \partial \bar{z}_{j}}=-\frac{4}{\pi} \iint_{D(z)} \frac{\partial^{2} g_{a}(z, w)}{\partial z_{i} \partial \bar{w}} \frac{\partial^{2} g_{a}(z, w)}{\partial \bar{z}_{j} \partial w} d u d v ; \\
& \frac{\partial^{2} H_{f}(z, a)}{\partial z_{i} \partial \bar{z}_{j}}=-\frac{2}{\pi} \iint_{D(z)}\left\{\frac{\partial^{2} g_{a}(z, w)}{\partial z_{i} \partial \bar{w} H_{f}(z, w)} \frac{\partial^{2} g_{a}(z, w)}{\partial \bar{z}_{j} \partial w} \frac{\partial^{2} H_{f}(z, w)}{\partial \bar{z}_{j} \partial w}\right\} d u d v ; \\
& \frac{\partial^{2}}{\partial z_{i} \partial \bar{z}_{j}}\left\{\iint_{D(z)}\left|\frac{\partial H_{f}(z, w)}{\partial w}\right|^{2} d u d v\right\}=\iint_{D(z)} \frac{\partial^{2} H_{f}(z, w)}{\partial z_{i} \partial \bar{w}} \frac{\partial^{2} H_{f}(z, w)}{\partial \bar{z}_{j} \partial w} d u d v .
\end{aligned}
$$

Le fait m'intéresse que la première formule de variation d'ordre deux pour les variations analytiques a de la ressemblance avec celle d'Hadamard d'ordre un pour les variations générales (p. 519 de [4]):

$$
\delta g_{a}(b)=-\frac{1}{2 \pi} \int_{C} \frac{\partial g_{a}(w)}{\partial n} \frac{\partial g_{b}(z, w)}{\partial n} \delta n d s
$$

Hadamard (p. 547 de [4]) en a obtenu une équation aux derivées fonctionnelles suivante à laquelle $\Phi(a, b)=\partial^{2} g_{a}(b) / \partial \bar{a} \partial b$ satisfait:

$$
\delta \Phi(a, b)=-\frac{1}{2 \pi} \int_{C} \Phi(a, w) \Phi(w, b) \delta n d s,
$$

qui est étudiée par P. Lévy [7].

On montre dans le présent mémoire, d'après les formules ci-dessus, les résultats suivants: on toujours suppose que $z \rightarrow D(z)$ où $z \in \Delta$ soit variation analytique.

(1) $K(z, w)|d w|^{2}$ étant la métrique de Bergmann (p. 33 de [3]) sur chaque $D(z)$, le coefficient $K(z, w)$ est fonction logarithmiquement pluri-sousharmonique par rapport à $z$ dans $\Delta$ (cf. le corollaire 3 et le théorème 1 de $\S 4$ );

(2) S'il existe $\mu$ points $\left\{a_{i}\right\}$ tels que $\mu \geqq 2$ et $\lambda\left(z, a_{i}\right)$ soit pluri-harmonique pour $z \in \Delta$, alors notre variation est équivalente à $z \rightarrow \tilde{R}(z)$ où $z \in \Delta$ telle qu'il existe un ouvert $R$ au-dessus du plan et $\mu$ points $\left\{A_{i}\right\}$ de $R$ de façon que $\tilde{R}(z)$ $(z \in \Delta)$ s'étale au-dessus de $R$ sans point frontière relatif de multiplicité $m$, et chaque $A_{i}$ soit point de ramification d'ordre $m-1$ de $\tilde{R}(z)$ (voir le lemme 3 de $\S 4)$; 
(3) Supposons que $n \geqq 2$ et la caractéristique d'Euler de $D(0)$ soit $\geqq 1$. $\mathrm{Si}$, pour tout $z\left(=\left(z_{1}, \cdots, z_{n-1}, z_{n}\right)\right)$ fixé dans $\Delta, D\left(\left(z_{1}, \cdots, z_{n-1}, z_{n}\right)\right)$ est équivalente à $D\left(\left(z_{1}, \cdots, z_{n-1}, 0\right)\right)$ comme surface de Riemann, alors notre variation est équivalente à $\left(z_{1}, \cdots, z_{n-1}, z_{n}\right) \rightarrow D\left(\left(z_{1}, \cdots, z_{n-1}, 0\right)\right)$ où $z \in \Delta$ (cf. le théorème 4 de $\S 9)$;

(4) $\Phi(a, b)=\partial^{2} g_{a}(b) / \partial \bar{a} \partial b$ satisfait à l'équation aux dérivées fonctionnelles (voir (b) de $\S 8)$ :

$$
\partial \bar{\partial} \Phi(a, b)=\frac{4}{\pi^{2}} \int_{C} \int_{C}\left(\operatorname{Re}\left\{\Phi\left(w_{1}, w_{2}\right) \partial \bar{w}_{1} \partial w_{2}\right\}\right) \Phi\left(a, w_{1}\right) \Phi\left(w_{2}, b\right) d w_{1} d \bar{w}_{2} .
$$

\section{§1. Définition de variation analytique}

Considérons un triple $(\mathfrak{S}, \Delta, \pi)$ tel que $\mathfrak{D}$ soit une variété complexe de dimension $n+1, \Delta\left(=\Delta_{1} \times \cdots \times \Delta_{n}\right)$ où $\Delta_{i}=\left\{\left|z_{i}\right|<\rho_{i}\right\}$ un polydisque dans l'espace de $n$ variable complexes $z_{1}, \cdots, z_{n}$, et $\pi$ une application analytique de $\mathfrak{D}$ sur $\Delta$ telle que $\pi$ soit submersion surjective, et pour tout $z \in \Delta$ la fibre $\pi^{-1}(z)$ soit irreductible. Pour $K$ un sous-ensemble quelconque de $\mathfrak{D}$, et $z$ de $\Delta$, on écrit $K(z)=\pi^{-1}(z) \cap K$. Toute fibre $\mathfrak{D}(z)$ munie de la structure induite par celle de (⿹) est une surface de Riemann d'une variable complexe. Etant donnés deux triples $\left(\mathfrak{D}_{i}, \Delta, \pi_{i}\right)(i=1,2)$, s'il existe isomorphisme analytique $\varphi$ de $\mathfrak{D}_{1}$ sur $\mathfrak{I}_{2}$ tel que $\pi_{2} \circ \varphi=\pi_{1}$, alors ils s'appellent équivalents.

Supposons que un triple (D), $\Delta, \pi$ ) satisfasse à la

Condition $(L):$ Il existe un autre triple $(\hat{D}, \Delta, \hat{\pi})$ tel que

(L1) $\mathfrak{D} \subset \mathfrak{D}$ et $\mathfrak{D}(z) \subset \mathfrak{D}(z)$ pour tout $z$ dans $\Delta ;$

(L2) la frontière $\partial \mathfrak{D}$ de $\mathfrak{D}$ dans $\mathfrak{\mathfrak { D }}$ consiste en Analytische Hyperflächenstuck.

S'il n'y a pas d'ambiquité, on abrège $\hat{\pi}=\pi$. Par définition (voir p. $28 \mathrm{de}$ [2]), (L2) signifie que, pour $P_{0} \in \partial \mathfrak{D}$, il existe un voisinage $\mathscr{V}$ de $P_{0}$ dans $\hat{\mathfrak{D}}$ et une fonction à valeur complexe $h(P, \zeta)$ analytique au sens réel définie dans $\mathscr{V} \times l$ où $l$ est un arc dans le plan $\zeta$ tel que, pour $\zeta$ fixé $\operatorname{sur} l, h(P, \zeta)$ soit analytique i.e., holomorphe dans $\mathscr{V}$ et que $\partial \mathscr{P} \cap \mathscr{V}=\{P \in \mathscr{V}: h(P, \zeta)=0$ où $\zeta \in$ l\}. Il en vient, pour tout $z \in \Delta,(\partial \mathscr{D})(z)=\partial(\mathfrak{D}(z))$, le second membre étant la frontière de $\mathfrak{D}(z)$ dans $\mathfrak{D}(z)$. Sous la condition $(L)$, la correspondance

$$
z \longrightarrow \mathfrak{D}(z) \text { où } z \in \Delta
$$

s'appelle variation analytique de surface de Riemann à bord $\mathfrak{S}(z)$. On donne un exemple simple. Soit $n=1, \Delta=\{|z|<1 / 2\}, \hat{\mathfrak{D}}=\Delta \times P^{1}$ où $\boldsymbol{P}^{1}=\{|w| \leqq \infty\}$, et $\mathfrak{D}=\left\{(z, w) \in \Delta \times \boldsymbol{P}^{1}:|(w-1)(w+1)|>|1+z|\right\}$. Puisqu'on peut prendre $h(z, w, \zeta)$ $=(w-1)(w+1)-(1+z) \zeta$ où $(z, w) \in \mathscr{V}=\mathfrak{D}$ et $\zeta \in l=\{|\zeta|=1\}$, on a une variation 
analytique $z \rightarrow \mathfrak{D}(z)$ où $z \in \Delta$ telle que $\partial \mathfrak{D}(z)$ se varie de façon que pour $z \in \Delta \cap$ $\{|1+z|<1\}$ (resp. $z \in \Delta \cap\{|1+z|=1\}$; resp. $z \in \Delta \cap\{|1+z|>1\}) \partial \Im(z)$ consiste en deux courbes fermées (resp. la lemniscate avec points fixés $\{-1,1\}$; resp. une courbe fermée) sur le plan $w$.

On soumet le triple $(\mathfrak{D}, \Delta, \pi)$ satisfaisant à la condition $(L)$ la

Condition $(T)$ : Le triple (D), $\Delta, \pi)$ est topologiquement trivial.

C'est-à-dire, il existe un homéomorphisme $\varphi$ de $\mathfrak{D}$ sur l'espace produit $\Delta \times \mathfrak{D}(0)$ tel que $p \circ \varphi=\pi$ où $p$ est la projection de $\Delta \times \mathfrak{D}(0)$ sur $\Delta$. Pour $z \in \Delta, \partial \mathfrak{D}(z)$ consiste un nombre fini $q$, indépendant de $z \in \Delta$, de courbes fermées sur $\mathfrak{D}(z)$ et le genre de $\mathfrak{D}(z)$ comme surface de Riemann est $p$, indépendant de $z \in \Delta$. On dit que (ID, $\Delta, \pi)$ est de type topologique $(p, q)$.

Soit $P_{0} \in \mathfrak{D} \cup \partial \mathfrak{D}$ tel que $\pi\left(P_{0}\right)=z_{0}$. On peut prendre par submersion de $\hat{\pi}$ des coordonnées locales d'un voisinage de $P_{0}$ dans $\hat{D}$ de la forme $(z, w) \in \Delta_{0} \times \delta$ telles que $\Delta_{0}$ soit un sous-polydisque de $\Delta$ de centre $z_{0}, \delta$ un disque $\{|w|<\tau\}$ du plan $w$ et $P_{0}$ corresponde à $\left(z_{0}, 0\right)$. On appelle telles $(z, w) \in \Delta_{0} \times \delta, \pi$-coordonnées de $P_{0}$.

Il est bien connu grâce à E. E. Levi (p. 83 de [6]) que les conditions $(L)$ et $(T)$ pour le triple $(\mathfrak{D}, \Delta, \pi)$ sont équivalentes à celle $(T)$ et la

Condition $\left(L^{\prime}\right)$ : Il existe une fonction à valeur réelle $F$ analytiue au sens réel dans $\mathfrak{D}$ telle que

$\left(L^{\prime} 1\right) \quad \mathfrak{D}=\{P \in \mathfrak{D}: F(P)>0\}$ et $\partial \mathfrak{D}=\{P \in \mathfrak{D}: F(P)=0\}$;

$\left(L^{\prime} 2\right)$ étant donné $P_{0} \in \partial \mathfrak{D}$ et $\Delta_{0} \times \delta$ des $\pi$-coordonnées de $P_{0}$, il vienne $(\partial F / \partial w)(z, w) \neq 0$ pour aucun point $(z, w) \in \partial \mathfrak{S} \cap\left(\Delta_{0} \times \delta\right)$, et la forme de Levi pour $i, j=1, \cdots, n$,

$$
\begin{aligned}
L_{i j}(z, w)= & \frac{\partial^{2} F}{\partial z_{i} \partial \bar{z}_{j}}\left|\frac{\partial F}{\partial w}\right|^{2}-\left\{\frac{\partial^{2} F}{\partial z_{i} \partial \bar{w}} \frac{\partial F}{\partial \bar{z}_{j}} \frac{\partial F}{\partial w}+\frac{\partial^{2} F}{\partial \bar{z}_{j} \partial w} \frac{\partial F}{\partial z_{i}} \frac{\partial F}{\partial \bar{w}}\right\} \\
& +\frac{\partial^{2} F}{\partial w \partial \bar{w}} \frac{\partial F}{\partial z_{i}} \frac{\partial F}{\partial \bar{z}_{j}}
\end{aligned}
$$

soint nulle pour $(z, w) \in \partial \mathfrak{D} \cap\left(\Delta_{0} \times \delta\right)$.

Comme d'habitude, on appelle une application analytique $\Xi$ de $\Delta_{0}(\subset \Delta)$ dans $\hat{\mathfrak{D}}$ telle que $\pi \circ \boldsymbol{E}(z)=z$ pour $z \in \Delta_{0}$, section analytique de $\mathfrak{D}$ sur $\Delta_{0}$, et on

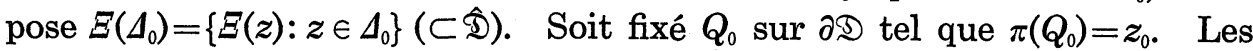
conditions $(L),(T)$ entraînent qu'il existe une et une seule section analytique $\Xi$ de $\hat{D}$ sur tout $\Delta$ telle que $\Xi$ passe par $Q_{0}$ i.e., $E\left(z_{0}\right)=Q_{0}$, et $\Xi(\Delta) \subset \partial D$. On écrit telle $\xi, E\left(Q_{0}, \cdot\right)$. Il s'ensuit la

Remarque 1. (1) pour tout $Q \in \partial \mathfrak{D}$ et tout $z \in \Delta$, on a $\boldsymbol{E}(Q, \cdot)=$ $\Xi(E(Q, z), \cdot) ;(2)$ pour $z_{0}$ fixé dans $\Delta$, on a pour $z \in \Delta$ 


$$
\begin{aligned}
& \partial \mathfrak{D}=\cup_{Q \in \partial \Phi\left(z_{0}\right)} \Xi(Q, \Delta) \quad \text { (l'union disjointe); } \\
& \partial \supseteqq(z)=\left\{\Xi(Q, z): Q \in \partial \supseteqq\left(z_{0}\right)\right\} ;
\end{aligned}
$$

(3) pour $z_{0}, z_{1} \in \Delta, \partial \mathfrak{D}\left(z_{0}\right)$ corresponde homéomorphiquement à $\partial \mathfrak{D}\left(z_{1}\right)$ de façon que $Q_{0}\left(\in \partial \mathfrak{D}\left(z_{0}\right)\right) \rightarrow Q_{1}=\Xi\left(Q_{0}, z_{1}\right)\left(\in \partial \mathfrak{D}\left(z_{1}\right)\right)$, et son inverse est donc $Q_{1} \rightarrow Q_{0}=$ $E\left(Q_{1}, z_{0}\right)$.

\section{§2. Calcul élémentaire}

Soit $(\mathfrak{D}, \Delta, \pi)$ un triple satisfaisant aux conditions $(L),(T)$. Soit $\mathscr{I}$ une partie de $\partial \mathfrak{D}$ consistant en nombre fini d'ouverts connexes de $\partial \mathfrak{D}$ telle que, pour tout $Q \in \mathscr{I}, \Xi(Q, \Delta) \subset \mathscr{I}$. Pour $z_{0}$ fixé dans $\Delta$, il s'ensuit que $\mathscr{I}\left(z_{0}\right)$ consiste en nombre fini d'arcs ou de contours de $\partial \mathfrak{S}\left(z_{0}\right)$, et pour $z \in \Delta$

$$
\mathscr{I}=\bigcup_{Q \in \mathscr{S}\left(z_{0}\right)} E(Q, \Delta) \quad \text { (l'union disjointe); }
$$

$$
\mathscr{I}(z)=\left\{\boldsymbol{E}(\boldsymbol{Q}, z): Q \in \mathscr{I}\left(z_{0}\right)\right\} \text {. }
$$

Soit donnée une fonction à valeur complexe $f$ analytique au sens réel définie sur $\mathscr{I}$ satisfaisant à la

Condition $(F):$ La restriction de $f$ sur la section analytique quelconque $\Xi$ de $\hat{D}$ sur $\Delta$ telle que $\Xi(\Delta) \subset \mathscr{I}$, soit pluri-harmonique, c'est-à-dire, $\partial^{2} f(E(z)) /$ $\partial z_{i} \partial \bar{z}_{j}=0(i, j=1, \cdots, n)$ pour $z \in \Delta$.

Soit $z_{0}$ fixé dans $\Delta$. En posant

$$
f_{0}(z, Q)=f(\Xi(Q, z)) \quad \text { pour } Q \in \mathscr{I}\left(z_{0}\right)
$$

la donnée de $f$ ci-dessus est équivalente à celle d'une fonction $f_{0}(z, Q)$ sur l'ensemble produit $\Delta \times \mathscr{I}\left(z_{0}\right)$ à valeur complexe et analytique au sens réel telle que $f_{0}(z, Q)$ soit, pour $Q$ fixé sur $\mathscr{I}\left(z_{0}\right)$, pluri-harmonique par rapport à $z \in \Delta$. On en définit une fonction $\partial f / \partial z_{i}(i=1, \cdots, n)$ sur $\mathscr{I}$ de façon que, pour tout $Q \in \mathscr{I}$ avec $\pi(Q)=c,\left(\partial f / \partial z_{i}\right)(Q)=\left\{\partial f(\Xi(Q, z)) / \partial z_{i}\right\}_{z=c}$. On a évidemment que $\left(\partial f / \partial z_{i}\right)(Q)=\left(\partial f_{0} / \partial z_{i}\right)\left(c, Q_{0}\right)$ tel que $Q_{0}=\boldsymbol{\Xi}\left(Q, z_{0}\right)$. Cette fonction $\partial f / \partial z_{i}$ sur $\mathscr{I}$ aussi satisfait, avec $f$, à la condition $(F)$.

Considérons une fonction $u(P)$ définie dans un voisinage $\mathscr{V}$ de $\mathscr{I}$ dans $\hat{\mathfrak{D}}$ soumise à la

Condition $(H): \quad u(P)$ est à valeur complexe et analytique au sens réel telle que

(H1) $u=f$ sur $\mathscr{I}$;

(H2) la restriction de $u(P)$ sur chaque fibre $\mathscr{V}(z)$ où $z \in \Delta$ soit harmonique. 
Soit $z_{0}=\left(z_{01}, \cdots, z_{0 n}\right) \in \Delta$. Il existe d'après les conditions $(L),(T)$ un voisinage de Stein $\mathfrak{U}$ de $\mathfrak{D}\left(z_{0}\right) \cup \partial \mathfrak{D}\left(z_{0}\right)$ dans $\mathfrak{D}$ tel que $\mathfrak{U}$ se réalise comme variété complexe de dimension $n+1$, un ouvert connexe $U$ multivalent étalé audessus du polycylindre $\Delta^{\prime} \times C$ où $\Delta^{\prime}$ est un sous-polydisque de $\Delta$ de centre $z_{0}$ et $C$ signifie le plan $w$, tel que $U$ n'ait aucune surface de ramification. Si l'on prend plus petit polydisque $\Delta_{0}$ de centre $z_{0}$ que $\Delta^{\prime}$, le sous-ensemble $\pi^{-1}\left(\Delta_{0}\right)$ $\cap(\mathfrak{D} \cup \partial \mathfrak{D})\left(=(\mathfrak{D} \cup \partial \mathfrak{D}) \mid \Delta_{0}\right)$ de $\mathfrak{D}$ est complètement contenu dans $\mathfrak{H}$. Ecrivons $D$ la partie de $U$ correspondante à celle $\pi^{-1}\left(\Delta_{0}\right) \cap D\left(=D \mid \Delta_{0}\right)$ de $\mathfrak{H}$. Alors le triple $\left(\mathfrak{D}\left|\Delta_{0}, \Delta_{0}, \pi\right| \Delta_{0}\right)$ est équivalent à $\left(D, \Delta_{0}, p\right)$ muni de la première projection $p$. Il s'ensuit que toute $\mathfrak{S}(z)\left(z \in \Delta_{0}\right)$ peut s'identifier comme surface de Riemann avec l'ouvert connexe $p^{-1}(z)=D(z)$ étalé au-dessus du plan $w$ satisfaisant à la

Condition $(R): \quad D(z)$ y s'étale multivalent en nombre fini de façon que

(R1) $D(z)$ n'ait aucun point de ramification;

(R2) la frontière $\partial D(z)$ de $D(z)$ consiste en nombre fini $q$ (indépendant de $z \in \Delta_{0}$ ) courbes fermées analytiques au sens réel;

(R3) $\partial D(z)=\left\{\xi_{0}(z, \zeta): \zeta \in \partial D\left(z_{0}\right)\right\}$ telle que $\xi_{0}\left(z_{0}, \zeta\right)=\zeta$ et $\xi_{0}(z, \zeta)$ soit, pour tout $\zeta$ fixé sur $\partial D\left(z_{0}\right)$, fonction analytique par rapport à $z$ dans $\Delta_{0}$.

On abrège $C=\partial D\left(z_{0}\right)$, et $I\left(z_{0}\right)$ la partie de $C$ corresponde à $\mathscr{I}\left(z_{0}\right)$. En général, $P($ resp. $Q)$ étant le point de $\mathfrak{H}$ (resp. $\left.\partial \mathfrak{D}\left(z_{0}\right)\right)$ tel que $\pi(P)=z$, on écrit $(z, w)$ (resp. $\zeta)$ celui de $U\left(\right.$ resp. $\left.\partial D\left(z_{0}\right)\right)$ correspondant à $P$ (resp. $Q$ ) tel que $w$ $\in U(z)$. Alors $\xi_{0}(z, \zeta)$ de $(R 3)$ corresponde à $\xi(Q, z)$ de $(*)$. On écrit $u(z, w)$ $=u(P)$. La fonction $f$ sur $\mathscr{I}$ définit celle $f_{0}$ sur le produit $\Delta_{0} \times I\left(z_{0}\right)$ de façon que $f_{0}(z, \zeta)=f(\Xi(z, Q))$. Il vient évidemment que $f_{0}(z, \zeta)=f_{0}(z, Q)$ qui est définie en $(* *)$, et $f_{0}(z, \zeta)$ est, pour $\zeta$ fixé sur $I\left(z_{0}\right)$, pluri-harmonique par rapport à $z \in$ $\Delta_{0}$. D'après $(H 1)$, on a alors

$$
u\left(z, \xi_{0}(z, \zeta)\right)=f_{0}(z, \zeta)
$$

pour $(z, \zeta) \in \Delta_{0} \times I\left(z_{0}\right)$. Par $z_{i}$-différentielle $(i=1, \cdots, n)$ de (2.1) on obtient d'après $(R 3)$

$$
\frac{\partial u}{\partial z_{i}}\left(z, \xi_{0}(z, \zeta)\right)+\frac{\partial u}{\partial w}\left(z, \xi_{0}(z, \zeta)\right) \frac{\partial \xi_{0}}{\partial z_{i}}(z, \zeta)=\frac{\partial f_{0}}{\partial z_{i}}(z, \zeta)
$$

pour $(z, \zeta) \in \Delta_{0} \times I\left(z_{0}\right)$, et en particulier

$$
\frac{\partial u}{\partial z_{i}}=\frac{\partial f_{0}}{\partial z_{i}}-\frac{\partial u}{\partial w} \frac{\partial \xi_{0}}{\partial z_{i}} \quad \text { sur }\left\{z_{0}\right\} \times I\left(z_{0}\right) .
$$

Pareillement on a

$$
\frac{\partial u}{\partial \bar{z}_{j}}=\frac{\partial f_{0}}{\partial \bar{z}_{j}}-\frac{\partial u}{\partial \bar{w}}\left(\overline{\frac{\partial \xi_{0}}{\partial z_{j}}}\right) \quad \text { sur }\left\{z_{0}\right\} \times I\left(z_{0}\right) .
$$


Par $\bar{z}_{j}$-différentielle de (2.2), on obtient d'après $(H 2),(F)$ et $(R 3)$

$$
\begin{gathered}
\frac{\partial^{2} u}{\partial \bar{z}_{j} \partial z_{i}}\left(z, \xi_{0}(z, \zeta)\right)+\frac{\partial^{2} u}{\partial z_{i} \partial \bar{w}}\left(z, \xi_{0}(z, \zeta)\right)\left(\overline{\left.\frac{\partial \xi_{0}}{\partial z_{j}}(z, \zeta)\right)}\right) \\
+\frac{\partial^{2} u}{\partial \bar{z}_{j} \partial w}\left(z, \xi_{0}(z, \zeta)\right) \frac{\partial \xi_{0}}{\partial z_{i}}(z, \zeta)=0
\end{gathered}
$$

pour $(z, \zeta) \in \Delta_{0} \times I\left(z_{0}\right)$, et en particulier

$$
\frac{\partial^{2} u}{\partial \bar{z}_{j} \partial z_{i}}=-\left\{\frac{\partial^{2} u}{\partial z_{i} \partial \bar{w}}\left(\overline{\frac{\partial \xi_{0}}{\partial z_{j}}}\right)+\frac{\partial^{2} u}{\partial \bar{z}_{j} \partial w} \frac{\partial \xi_{0}}{\partial z_{i}}\right\} \quad \text { sur }\left\{z_{0}\right\} \times I\left(z_{0}\right) .
$$

D'après (2.3) et (2.4), il vient sur $\left\{z_{0}\right\} \times I\left(z_{0}\right)$

$$
\frac{\partial^{2} u}{\partial \bar{z}_{j} \partial z_{i}}=\frac{\left(\partial^{2} u / \partial z_{i} \partial \bar{w}\right)\left(\left(\partial u / \partial \bar{z}_{j}\right)-\left(\partial f_{0} / \partial \bar{z}_{j}\right)\right)}{\partial u / \partial \bar{w}}+\frac{\left(\partial^{2} u / \partial \bar{z}_{j} \partial w\right)\left(\left(\partial u / \partial z_{i}\right)-\left(\partial f_{0} / \partial z_{i}\right)\right)}{\partial u / \partial w}
$$

On sait ainsi que pour la variation analytique: $z \rightarrow \mathfrak{S}(z)$ où $z \in \Delta$, la condition $(F)$ pour $f$ et celle $(H)$ pour $u$ entraînent que la variation d'ordre deux de la forme $\delta_{i} \bar{\delta}_{j}$ sur $\mathscr{I}$ de $u$ s'exprime en celle d'ordre $u n$ de la forme $\bar{\delta}_{i}$ ou $\bar{\delta}_{j}$ des $u$, $\partial u / \partial w$ et $f$. Etant donnée, en outre, une autre fonction $f_{1}$ sur $\mathscr{I}$ satisfaisant à la condition $(F)$ et une fonction $u_{1}(P)$ définie dans $\mathscr{V}$ satisfaisant à la condition $(H)$ pour $f=f_{1}$, on a aussi sur $\left\{z_{0}\right\} \times I\left(z_{0}\right)$

$$
\begin{aligned}
\frac{\partial^{2} u}{\partial \bar{z}_{j} \partial z_{i}}= & \frac{\left(\partial^{2} u / \partial z_{i} \partial \bar{w}\right)\left(\left(\partial u_{1} / \partial \bar{z}_{j}\right)-\left(\partial f_{10} / \partial \bar{z}_{j}\right)\right)}{\partial u_{1} / \partial \bar{w}} \\
& +\frac{\left(\partial^{2} u / \partial \bar{z}_{j} \partial w\right)\left(\left(\partial u_{1} / \partial z_{i}\right)-\left(\partial f_{10} / \partial z_{i}\right)\right)}{\partial u_{1} / \partial w}
\end{aligned}
$$

Or, puisque $\xi_{0}(z, \zeta)$ de $(R 3)$ est analytique au sens réel pour $(z, \zeta) \in \Delta_{0} \times \partial D\left(z_{0}\right)$ et analytique pour $z \in \Delta_{0}$, il s'ensuit la

Remarque 2 (p. 30 de [2]). Soit (P, $\Delta, \pi)$ un triple vérifiant aux conditions $(L),(T)$. Soit $P_{0} \in \partial \mathfrak{D}$ tel que $\pi\left(P_{0}\right)=z_{0}$. Il existe alors des $\pi$-coordonnées particulières $\Delta_{0} \times \delta$ où $z_{0} \in \Delta_{0} \subset \Delta$ et $\delta=\{|w|<1\}$, d'un voisinage $\mathscr{V}$ de $P_{0}$ dans $\hat{\mathfrak{D}}$ tel que $P_{0}$ (resp. $\mathscr{V} \cap \mathfrak{D} ;$ resp. $\mathscr{V} \cap \partial \mathfrak{D} ;$ resp. $\xi_{0}(z, \zeta)$ de $(R 3)$ ) corresponde à $(z, 0)$ (resp. $\Delta_{0} \times\{w \in \delta ; \operatorname{Im} w>0\} ;$ resp. $\Delta_{0} \times\{w \in \delta: \operatorname{Im} w=0\} ; \operatorname{resp} .(z, w(\zeta))$ tel que $\operatorname{Im} w(\zeta)=0$ et $w(\zeta)$ ne dépende pas de $\left.z \in \Delta_{0}\right)$.

Pour le calcul ultérieur, il faut une notation. Soit $P_{0} \in \mathfrak{S} \cup \partial \mathfrak{D}$ tel que $\pi\left(P_{0}\right)=z_{0}$, et $(z, w) \in \Delta_{0} \times \delta$ des $\pi$-coordonnées d'un voisinage $\mathscr{V}$ de $P_{0}$ dans $\mathfrak{D}$. Par définition, $\delta=\{|w|<\tau\}$ devient une coordonnée locale sur chaque surface de Riemann $\mathfrak{D}(z)\left(z \in \Delta_{0}\right)$. Maintenant, pour tout $z$ fixé dans $\Delta$, soit donnée une 1-forme méromorphe $\omega(z, \cdot)$ sur $\mathfrak{S}(z)$ satisfaisant à la condition $(d)$ : quand 
on désigne $\omega(z, \cdot)=h(z, w) d w$ pour $w \in \delta, h(z, w)$ soit fonction de classe $C^{\infty}$ sur $\Delta_{0} \times \delta$. Considérons la forme $\left(\partial h / \partial \bar{z}_{i}\right)(z, w) d w(i=1, \cdots, n)$ sur $\delta$. Elle ne dépend pas de choix ni de $P_{0} \in \mathfrak{S} \cup \partial \mathfrak{D}$ ni de $\pi$-coordonnées $(z, w) \in \Delta_{0} \times \delta$ de $P_{0}$. En effet, soit $P_{1} \in \mathfrak{D} \cup \partial \mathfrak{D}$ tel que $\pi\left(P_{1}\right)=z_{1}$ et $(z, W) \in \Delta_{1} \times \delta_{1}$ où $z_{1} \in \Delta_{1} \subset \Delta$ et $\delta_{1}$ $=\left\{|W|<\tau_{1}\right\}$, des $\pi$-coordonnées d'un voisinage $\mathscr{V}_{1}$ de $P_{1}$ dans $\mathfrak{D}$ telles que $\mathscr{V} \cap$ $\mathscr{V}_{1} \neq \phi$. Pour $z \in \Delta_{1}$, on a $\omega(z, \cdot)=H(z, W) d W$ sur $\delta_{1}$ telle que $H(z, W)$ soit méromorphe pour $W \in \delta_{1}$. Il y a une relation analytique $(z, w) \rightarrow(z, W(z, w))$ entre la partie correspondante à $\mathscr{V} \cap \mathscr{V}_{1}$ de $\Delta_{0} \times \delta$ et celle de $\Delta_{1} \times \delta_{1}$ et on y a $h(z, w)=H(z, W(z, w))(\partial W / \partial w)(z, w)$. Par $\bar{z}_{i}$-différentielle, il vient $\left(\partial h / \partial \bar{z}_{i}\right)(z, w)$ $=\left(\partial H / \partial \bar{z}_{i}\right)(z, W(z, w))(\partial W / \partial w)(z, w)$, ce qu'on désire. On a ainsi obtenu, sur chaque $\mathfrak{S}(z)$ où $z \in \Delta$, une nouvelle 1-forme méromorphe induite par $\omega(z, \cdot)=$ $h(z, w) d w$. Elle s'appelle $\bar{z}_{i}$-différentielle de $\omega(z, \cdot)$ et s'écrit

$$
\frac{\partial \omega}{\partial \bar{z}_{i}}(z, \cdot)=\frac{\partial h}{\partial \bar{z}_{i}}(z, w) d w
$$

Par le même procède, on aura $z_{j}$-différentielle $\partial^{2} \omega / \partial \bar{z}_{i} \partial \bar{z}_{j}$ de $\left(\partial \omega / \partial \bar{z}_{i}\right)(z, \cdot)$, et en général $\left(\partial^{N} \omega / \partial \bar{z}_{1}^{i_{1}} \cdots \partial \bar{z}_{n}^{i_{n}}\right)(z, \cdot)$ où $N=i_{1}+\cdots+i_{n}$.

On soumet de plus une 1-forme méromorphe $\omega(z, \cdot)=h(z, w) d w$ sur chaque $\mathfrak{D}(z)$ avec condition $(d)$ à la

Condition (e): Pour tout $z \in \Delta, \omega(z, \cdot)$ a des singularités aux points $\alpha_{k}(z)$ $(k=1, \cdots, \nu) \operatorname{sur} \mathfrak{D}(z) \cup \partial \mathfrak{D}(z)$ telles que

(e1) toute $\alpha_{k}: z(\in \Delta) \rightarrow \alpha_{k}(z)(\in(\mathfrak{D} \cup \partial \mathfrak{D})(z))$ détermine une section analytique de $\hat{\mathfrak{D}} \operatorname{sur} \Delta ;$

(e2) Soit $z_{0} \in \Delta$ et $\Delta_{0} \times \delta$ des $\pi$-coordonnées du point $\alpha_{k}\left(z_{0}\right)$ dans $\hat{D}$, et écrivons $\left(z, \alpha_{k}(z)\right)$ le point de $\Delta_{0} \times \delta$ correspondant à celui $\alpha_{k}(z)$ de $\ \cup \partial \supseteqq$. On a alors le développement dans $\Delta_{0} \times \delta$

$$
h(z, w)=\sum_{n=1}^{\infty} \frac{b_{n}(z)}{\left(w-\alpha_{k}(z)\right)^{n}}+\sum_{n=0}^{\infty} a_{n}(z)\left(w-\alpha_{k}(z)\right)^{n}
$$

tel que le coefficient $b_{n}(z)(n=1,2, \cdots)$ soit analytique pour $z$ dans $\Delta_{0}$.

Cette condition ne dépend pas de choix de $\pi$-coordonnées $\Delta_{0} \times \delta$ et on a $\partial h(z, w) / \partial \bar{z}_{i}=\sum_{n=0}^{\infty}\left(\partial a_{n} / \partial \bar{z}_{i}\right)\left(w-\alpha_{k}(z)\right)^{n}$, d'où $(\partial \omega / \partial \bar{z})(z, \cdot)$ est 1-forme analytique sans singularité sur chaque $\mathfrak{D}(z) \cup \partial \mathfrak{D}(z)$. Il vient le

LEMME 1. Etant données deux 1-formes méromorphes $\omega_{k}(z, \cdot)=h_{k}(z, w) d w$ $(k=1,2)$ sur $\mathfrak{S}(z) \cup \partial \mathfrak{S}(z)$ (où $z \in \Delta)$ satisfaisant aux conditions $(d)$ et $(e)$, l'intégrale mixte de la forme

$$
\left(\frac{\partial \omega_{1}}{\partial \bar{z}_{i}}(z, \cdot), \frac{\partial \omega_{2}}{\partial \bar{z}_{j}}(z, \cdot)\right)_{Ð(z)}=2 \iint_{Ð(z)} \frac{\partial h_{1}(z, w)}{\partial \bar{z}_{i}} \frac{\partial \bar{h}_{2}(z, w)}{\partial z_{\jmath}} d u d v
$$

où $w=u+\sqrt{-1} v(u, v$ étant réels), est finie pour $z$ fixé dans $\Delta$. 


\section{§3. Variations de fonctions de Green}

Soit $R$ une surface de Riemann à bord $\partial R$. Etant donné un point $P_{0}$ de $R$, il existe uniquement la fonction de Green $g_{P_{0}}(w)$ sur $R$ avec pôle (logarithmique) à $P_{0}$ telle que $g_{P_{0}}(w)$ soit harmonique sur $R-\left\{P_{0}\right\}, g_{P_{0}}(w)$ soit nulle sur $\partial R$ et $\{|w|<\tau\}$ étant une coordonnée locale d'un voisinage de $P_{0}$ dans $R$ tel que $P$ corresponde à $w=0$, elle y s'exprime

$$
g_{P_{0}}(w)=\log \frac{1}{|w|}+\lambda+\operatorname{Re}\left\{\sum_{n=1}^{\infty} a_{n} w^{n}\right\} .
$$

Le terme constant $\lambda$ est dit la constante de Robin de $R$ par rapport au point $P_{0}$ et à la coordonnée locale $\{|w|<\tau\}$. Quand on prend autre coordonnée locale $\left\{|W|<\tau_{1}\right\}$ d'un voisinage de $P_{0}$ et on écrit $\Lambda$ la constante de Robin par rapport à $P_{0}$ et à cette coordonnée, on a

$$
\lambda=\Lambda-\log \left|\frac{d W}{d w}(0)\right| .
$$

Soit $(\mathfrak{D}, \Delta, \pi)$ un triple satisfaisant aux conditions $(L),(T)$ de $\S 1$. Soit $\alpha: \Delta$ $\rightarrow \mathfrak{D}$ une section analytique de $\mathfrak{D}$ sur $\Delta$. Considérons sur chaque fibre $\mathfrak{D}(z)$ où $z \in \Delta$ la fonction de Green $g_{\alpha}(z, w)$ avec pole au point $\alpha(z)$.

FoRmule 1. Soit $\beta: \Delta \rightarrow \mathfrak{D}$ une section analytique de $\mathfrak{D}$ sur $\Delta$ telle que $\alpha(z)$ $\neq \beta(z)$ pour aucun $z$ de $\Delta$. On a alors pour $z=\left(z_{1}, \cdots, z_{n}\right)$ et $i, j=1, \cdots, n$,

$$
\frac{\partial^{2} g_{\alpha}(z, \beta(z))}{\partial z_{i} \partial \bar{z}_{j}}=-\frac{2}{\pi} \iint_{\Phi_{(z)}}\left(\frac{\partial^{2} g_{\alpha}}{\partial z_{i} \partial \bar{w}} \frac{\partial^{2} g_{\beta}}{\partial \bar{z}_{j} \partial w}+\frac{\partial^{2} g_{\alpha}}{\partial \bar{z}_{j} \partial w} \frac{\partial^{2} g_{\beta}}{\partial z_{i} \partial \bar{w}}\right) d u d v .
$$

DÉmonstration. Puisque $\alpha$ (resp. $\beta$ ) est section analytique de $\mathfrak{D}$ et $\partial \mathfrak{D}(z)$ se varie avec $z \in \Delta$ analytiquement au sens réel dans $\hat{D}$, la donnée de la 1forme méromorphe $\left(\partial g_{\alpha} / \partial w\right) d w$ (resp. $\left.\left(\partial g_{\beta} / \partial w\right) d w\right)$ ayant le pôle d'ordre un à $\alpha(z)$ (resp. $\beta(z)$ ) sur chaque $\mathfrak{D}(z)$ où $z \in \Delta$ vérifie aux conditions $(d),(e)$ de $\S 2$. Par le lemme 1, le second membre, comme celui premier, détermine donc une valeur complexe finie indépendante de triple quelconque équivalent à $(\mathscr{D}$, $\Delta, \pi)$.

Soit $z_{0} \in \Delta$. Il existe d'après $\S 2$ un petit polydisque $\Delta_{0}$ de centre $z_{0}$ dans $\Delta$ tel que le triple $\left(\mathscr{D}\left|\Delta_{0}, \Delta_{0}, \pi\right| \Delta_{0}\right)$ soit équivalent à $\left(D, \Delta_{0}, p\right)$ tel que $D(\subset U)$ soit un ouvert connexe multivalent étale au-dessus de $\Delta_{0} \times C$ où $C$ est le plan $w$, et $p$ est la projection de $D$ sur $\Delta_{0}$ et qu'il satisfasse à la condition $(R)$ de $\S 2$. On peut supposer d'après $\alpha(z) \neq \beta(z)$ pour $z \in \Delta$ qu'il vérifie de plus à la condition

(R4) l'ensemble analytique $\alpha\left(\Delta_{0}\right)$ (resp. $\beta\left(\Delta_{0}\right)$ ) dans $\mathfrak{D}$ corresponde au hyperplan $O=\Delta_{0} \times\{w=0\}$ (resp. $I=\Delta_{0} \times\{w=1\}$ ) dans $D$. 
D'après $(R 1)$, la coordonnée $w$ de $C$ se regarde celle locale d'un point quelconque de chaque surface de Riemann $\mathfrak{D}(z)$ où $z \in \Delta_{0}$. Pour facilité on en écrit

$$
g_{\alpha}(z, w)=g_{0}(z, w) \quad \text { et } \quad g_{\beta}(z, w)=g_{1}(z, w) .
$$

Puisque $\partial \mathfrak{D}$ est analytique au sens réel dans $\hat{D}$, le théorème de Fredholm entraîne que $g_{0}(z, w)$ (resp. $g_{1}(z, w)$ ) est analytique au sens réel par rapport à $(z, w)$ dans $D$ sauf $O$ (resp. $I)$. On a le développement dans un voisinage de $O$,

$$
g_{0}(z, w)=\log \frac{1}{|w|}+\lambda_{0}(z)+\operatorname{Re}\left\{\sum_{n=1}^{\infty} a_{n}(z) w^{n}\right\}
$$

pour $(z, w) \in \Delta_{0} \times \delta_{0}$ où $\delta_{0}=\left\{|w|<\tau_{0}\right\}$, et dans un voisinage de $I$,

$$
g_{1}(z, w)=\log \frac{1}{|w-1|}+\lambda_{1}(z)+\operatorname{Re}\left\{\sum_{n=1}^{\infty} b_{n}(z)(w-1)^{n}\right\}
$$

pour $(z, w) \in \Delta_{0} \times \delta_{1}$ où $\delta_{1}=\left\{|w-1|<\tau_{1}\right\}$. D'après $(R 1),(R 2)$, toute $g_{0}(z, w)$ (resp. $\left.g_{1}(z, w)\right)$ où $z \in \Delta_{0}$ se regarde une fonction harmonique par rapport à $w$ dans un voisinage de $D\left(z_{0}\right) \cup \partial D\left(z_{0}\right)$ dans $U\left(z_{0}\right)$ sauf le point $O \cap\left\{z=z_{0}\right\} \quad\left(=O\left(z_{0}\right)\right)$ (resp. $I \cap\left\{z=z_{0}\right\}\left(=I\left(z_{0}\right)\right)$ ). Si nécessaire, il suffit de reprendre assez petit $\Delta_{0}$. On abrège $C=\partial D\left(z_{0}\right)$ et elle est toujours positivement orientée à l'ouvert $D\left(z_{0}\right)$. Comme d'habitude,on a d'après la formule de Stokes sur $D\left(z_{0}\right)-\left\{O\left(z_{0}\right)\right.$, $\left.I\left(z_{0}\right)\right\}$, pour tout $z \in \Delta_{0}$,

$$
g_{0}(z, 1)-g_{0}\left(z_{0}, 1\right)=-\frac{1}{2 \pi} \int_{c} g_{0}(z, w) \frac{\partial g_{1}\left(z_{0}, w\right)}{\partial n_{w}} d s_{w},
$$

$\partial / \partial n_{w}$ étant la derivée normale extérieure de $C$ et $d s_{w}$ l'élément d'arc de $C$. D'après

$$
\frac{\partial}{\partial n_{w}} d s_{w}=\frac{1}{\sqrt{-1}}\left(\frac{\partial}{\partial w} d w-\frac{\partial}{\partial \bar{w}} d \bar{w}\right) \quad \text { le long de } C,
$$

et $g_{1}\left(z_{0}, w\right)=0$ sur $C$, on a

$$
\frac{\partial g_{1}\left(z_{0}, w\right)}{\partial w} d w+\frac{\partial g_{1}\left(z_{0}, w\right)}{\partial \bar{w}} d \bar{w}=0 \quad \text { le long de } C,
$$

il en vient pour $z \in \Delta_{0}$,

$$
g_{0}(z, 1)-g_{0}\left(z_{0}, 1\right)=\frac{\sqrt{-1}}{\pi} \int_{c} g_{0}(z, w) \frac{\partial g_{1}\left(z_{0}, w\right)}{\partial w} d w .
$$

$C$ étant indépendant de $z \in \Delta_{0}$, il en vient 


$$
\frac{\partial g_{0}(z, 1)}{\partial z_{i}}=\frac{\sqrt{-1}}{\pi} \int_{0} \frac{\partial g_{0}(z, w)}{\partial z_{i}} \frac{\partial g_{1}\left(z_{0}, w\right)}{\partial w} d w .
$$

Par $\bar{z}_{j}$-différentielle, il vient pour $z \in \Delta_{0}$

$$
\frac{\partial^{2} g_{0}(z, 1)}{\partial \bar{z}_{j} \partial z_{i}}=\frac{\sqrt{-1}}{\pi} \int_{C} \frac{\partial^{2} g_{0}(z, w)}{\partial \bar{z}_{j} \partial z_{i}} \frac{\partial g_{1}\left(z_{0}, w\right)}{\partial w} d w .
$$

En appliquant (2.8) pour $\mathscr{I}\left(z_{0}\right)=C: f=0 ; u=g_{0}(z, w) ; f_{1}=0 ; u_{1}=g_{1}(z, w)$, on a d'après (3.5),

$$
\begin{aligned}
& \left\{\frac{\partial^{2} g_{0}(z, 1)}{\partial \bar{z}_{j} \partial z_{i}}\right\}_{z=z_{0}} \\
& \quad=\frac{\sqrt{-1}}{\pi} \int_{C}\left\{\left(\frac{\left(\partial^{2} g_{0} / \partial \bar{z}_{j} \partial w\right)\left(\partial g_{1} / \partial z_{i}\right)}{\partial g_{1} / \partial w}+\frac{\left(\partial^{2} g_{0} / \partial z_{i} \partial \bar{w}\right)\left(\partial g_{1} / \partial \bar{z}_{j}\right)}{\partial g_{1} / \partial \bar{w}}\right) \frac{\partial g_{1}}{\partial w}\right\}_{\left(z_{0}, w\right)} d w \\
& \quad=\frac{\sqrt{-1}}{\pi} \int_{C}\left\{\frac{\partial^{2} g_{0}}{\partial \bar{z}_{j} \partial w} \frac{\partial g_{1}}{\partial z_{i}}\right\}_{\left(z_{0}, w\right)} d w-\left\{\frac{\partial^{2} g_{0}}{\partial z_{i} \partial \bar{w}} \frac{\partial g_{1}}{\partial \bar{z}_{j}}\right\}_{\left(z_{0}, w\right)} d \bar{w} .
\end{aligned}
$$

D'après (3.3), $\left(3.3^{\prime}\right)$, on a dans un voisinage de $O$ ou $I$ dans $D$,

$$
\begin{gathered}
\frac{\partial g_{1}}{\partial z_{i}}(z, w)=\frac{\partial \lambda_{1}(z)}{\partial z_{i}}+\frac{1}{2} \sum_{n=1}^{\infty}\left(\frac{\partial b_{n}(z)}{\partial z_{i}}(w-1)^{n}+\frac{\partial \overline{b_{n}(z)}}{\partial z_{i}}(\overline{w-1})^{n}\right) \\
\frac{\partial^{2} g_{0}}{\partial \bar{z}_{j} \partial w}(z, w)=\frac{1}{2} \sum_{n=1}^{\infty} n \frac{\partial a_{n}(z)}{\partial \bar{z}_{j}} w^{n-1} .
\end{gathered}
$$

Il s'ensuit que toutes les fonctions sous l'intégrale du troisième membre de (3.8) sont analytiques au sens réel sans singularité sur l'ouvert $D\left(z_{0}\right)$. Grâce à la formule de Stokes, on en a

$$
\begin{aligned}
& \left\{\frac{\partial^{2} g_{0}(z, 1)}{\partial \bar{z}_{j} \partial z_{i}}\right\}_{z=z_{0}} \\
& =\frac{\sqrt{-1}}{\pi} \iint_{D\left(z_{0}\right)} d\left(\left\{\frac{\partial^{2} g_{0}}{\partial \bar{z}_{j} \partial w} \frac{\partial g_{1}}{\partial z_{i}}\right\}_{\left(z_{0}, w\right)} d w\right)-d\left(\left\{\frac{\partial^{2} g_{0}}{\partial z_{i} \partial \bar{w}} \frac{\partial g_{1}}{\partial \bar{z}_{j}}\right\}_{\left(z_{0}, w\right)} d \bar{w}\right) \\
& =\frac{\sqrt{-1}}{\pi} \iint_{D\left(z_{0}\right)}\left\{\frac{\partial^{2} g_{0}}{\partial \bar{z}_{j} \partial w} \frac{\partial^{2} g_{1}}{\partial z_{i} \partial \bar{w}}\right\}_{\left(z_{0}, w\right)} d \bar{w} \wedge d w \\
& \quad-\left\{\frac{\partial^{2} g_{0}}{\partial z_{i} \partial \bar{w}} \frac{\partial^{2} g_{1}}{\partial \bar{z}_{j} \partial w}\right\}_{\left(z_{0}, w\right)} d w \wedge d \bar{w},
\end{aligned}
$$

car pour $z$ fixé dans $\Delta,\left(\partial g_{0} / \partial \bar{z}_{j}\right)(z, w)$ et $\left(\partial g_{1} / \partial z_{i}\right)(z, w)$ sont fonctions harmoniques par rapport à $w$ dans $D\left(z_{0}\right)$. On a enfin par $w=u+\sqrt{-1} v$ (u,v réels),

$$
\begin{aligned}
& \left\{\frac{\partial^{2} g_{0}(z, 1)}{\partial \bar{z}_{j} \partial z_{i}}\right\}_{z=z_{0}} \\
& \quad=-\frac{2}{\pi} \iint_{D\left(z_{0}\right)}\left\{\frac{\partial^{2} g_{0}}{\partial \bar{z}_{j} \partial w} \frac{\partial^{2} g_{1}}{\partial z_{i} \partial \bar{w}}+\frac{\partial^{2} g_{0}}{\partial z_{i} \partial \bar{w}} \frac{\partial^{2} g_{1}}{\partial \bar{z}_{j} \partial w}\right\}_{\left(z_{0}, w\right)} d u d v .
\end{aligned}
$$


D'après (3.2) et $g_{0}(z, 1)=g_{\alpha}(z, \beta(z))$ pour tout $z \in \Delta_{0}$, cette égalité n'est autre que la formule 1 pour $z=z_{0}$. $z_{0}$ étant quelconque de $\Delta$, la formule 1 est démontrée.

\section{§4. Variations de constante de Robin}

Soit $(\mathfrak{D}, \Delta, \pi)$ le triple satisfaisant aux conditions $(L),(T)$. Soit $\alpha$ une section analytique de $\mathfrak{D}$, et $g_{\alpha}(z, w)$ la fonction de Green de chaque $\mathfrak{D}(z)$ où $z \in \Delta$ avec pôle à $\alpha(z)$. Soit $z_{0} \in \Delta$ et $(z, w) \in \Delta_{0} \times \delta$ des $\pi$-coordonnées de $\alpha\left(z_{0}\right)$ dans $\mathfrak{D}, \Delta_{0}$ étant un polydisque de centre $z_{0}$ dans $\Delta$ et $\delta=\{|w|<\tau\}$. On désigne par même notation $(z, \alpha(z)) \in \Delta_{0} \times \delta$ le point correspondant à $\alpha(z) \in \mathfrak{D}(z)$ pour $z \in \Delta_{0}$. On a le développement

$$
g_{\alpha}(z, w)=\log \frac{1}{|w-\alpha(z)|}+\lambda_{\alpha}(z)+\operatorname{Re}\left\{\sum_{n=1}^{\infty} a_{n}(z)(w-\alpha(z))^{n}\right\}
$$

pour $(z, w) \in \Delta_{0} \times \delta$. La constante de Robin $\lambda_{\alpha}(z)$ de $\mathfrak{T}(z)$ par rapport à $\alpha(z)$ et à $\delta$ dépend de $\pi$-coordonnées $\Delta_{0} \times \delta$, mais, la différentielle de la forme $\partial^{2} \lambda_{\alpha}(z) / \partial z_{i} \partial \bar{z}_{j}(i, j=1, \cdots, n)$ n'en dépend pas. Car, $(z, W) \in \Delta_{1} \times \delta_{1}$ étant autres $\pi$-coordonnées de $\alpha\left(z_{0}\right)$ dans $\mathscr{P}$ où $\delta_{1}=\left\{|W|<\tau_{1}\right\}$, on écrit $\Lambda_{\alpha}(z)$ la constante de Robin de $\mathfrak{D}(z)$ par rapport à $\alpha(z)$ et à $\delta_{1}$. Il y a la relation analytique $z=z$, $W=W(z, w)$ d'un voisinage de $\left(z_{0}, \alpha\left(z_{0}\right)\right)$ dans $\Delta_{0} \times \delta$ sur celui dans $\Delta_{1} \times \delta_{1}$, et, d'après (3.1), la relation $\Lambda_{\alpha}(z)=\lambda_{\alpha}(z)+\log |(\partial W / \partial w)(z, \alpha(z))|$ pour $z \in \Delta_{0} \cap \Delta_{1}$. Puisque $\alpha(z)$ est analytique, on a $\partial^{2} \Lambda_{\alpha}(z) / \partial z_{i} \partial \bar{z}_{j}=\partial^{2} \lambda_{\alpha}(z) / \partial z_{i} \partial \bar{z}_{j}$, ce qu'on demande. Il s'ensuit par la même méthode que la formule 1 que $\partial^{2} \lambda_{\alpha} / \partial z_{i} \partial \bar{z}_{j}$ est calculée dans la

Formule 2. Soit (D, $\Delta, \pi)$ le triple vérifiant aux conditions $(L),(T)$. Pour la section analytique $\alpha$ de $\mathfrak{D}$ sur $\Delta$, il vient

$$
\frac{\partial^{2} \lambda_{\alpha}(z)}{\partial z_{i} \partial \bar{z}_{j}}=-\frac{4}{\pi} \iint_{D(z)} \frac{\partial^{2} g_{\alpha}}{\partial z_{i} \partial \bar{w}} \frac{\partial^{2} g_{\alpha}}{\partial \bar{z}_{j} \partial w} d u d v
$$

Dans cette formule, posons en particulier $i=j$. On sait que $\partial^{2} \lambda_{\alpha} / \partial z_{i} \partial \bar{z}_{i}=0$ dans $\Delta$ si et seulement si $\left(\partial^{2} g_{\alpha} / \partial \bar{z}_{i} \partial w\right) d w=0$ sur chaque $\mathfrak{T}(z)$ où $z \in \Delta$, i.e., $\left(\partial g_{\alpha}(z, w) / \partial w\right) d w$ est analytique pour $z \in \Delta$. D'après

$$
g_{\alpha}(z, w)=2 \operatorname{Re} \int_{\delta\left(Q_{0}, z\right)}^{w} \frac{\partial g_{\alpha}(z, w)}{\partial w} d w
$$

où $Q_{0}$ est un point fixé sur $\partial \mathfrak{S}(0)$, il en résulte la

Remarque 3. Sous la même notation ci-dessus, la pluri-harmonicité de $\lambda_{\alpha}(z)$ pour $z \in \Delta$ ne dépend pas de choix de $\pi$-coordonnées de $\mathfrak{D}$. De plus, $\lambda_{\alpha}(z)$ 
est pluri-harmonique pour $z \in \Delta$ si et seulement si $g_{\alpha}(z, w)$ est pluri-harmonique pour $(z, w) \in \mathfrak{D}-\alpha(\Delta)$.

Soient $\left(a_{1}, \cdots, a_{n}\right) n$ nombres complexes quelconques. On a, par la formule 2 ,

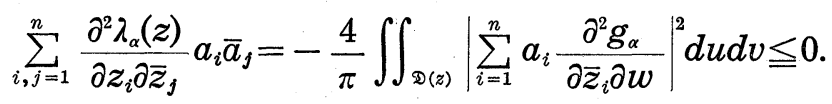

CoRollaIre 1. Sous la même configuration que la formule 2, la matrice $\left[\partial^{2} \lambda_{\alpha}(z) / \partial z_{i} \partial \bar{z}_{j}\right]_{i, j=1, \ldots, n}$ est non-positive définitive pour $z \in \Delta$, i.e., $\lambda_{\alpha}(z)$ est fonction pluri-surharmonique pour $z \in \Delta$.

Remarque 4. Cette propriété de $\lambda_{\alpha}(z)$ est vraie (p. 72 de [14]) pour le triple $(\mathfrak{D}, \Delta, \pi)$ vérifiant au lieu de $(T),(L)$ à la condition plus faible telle que (S) soit variété de Stein. La signification de ce fait est traitée dans l'énoncé de T. Nishino [9].

Soit, maintenant, $R$ une surface de Riemann à bord et $\delta=\{|w|<\tau\}$ une coordonnée locale d'un voisinage $\mathscr{V}$ dans $R$. Au point $w_{1}$ de $\delta$, on écrit $P_{1}$ celui correspondant de $\mathscr{V}$. On dénote par $g\left(w_{1}, w\right)$ (resp. $\left.\lambda\left(w_{1}\right)\right)$ la fonction de Green sur $R$ avec pôle à $P_{1}$ (resp. la constante de Robin par rapport à $P_{1}$ et à $\delta)$. En prenant, comme $(\mathfrak{D}, \Delta, \pi)($ resp. $\alpha)$ de la formule $2,(\delta \times R, \delta, p), p$ étant la première projection (resp. $\left.\alpha: w_{1} \in \delta \rightarrow\left(w_{1}, P_{1}\right) \in \delta \times R\right)$, on a le

Corollatre 2 (p. 213 de N. Suita [12]).

$$
\frac{\partial^{2} \lambda\left(w_{1}\right)}{\partial w_{1} \partial \bar{w}_{1}}=-\frac{4}{\pi} \iint_{R}\left|\frac{\partial^{2} g\left(w_{1}, w\right)}{\partial \bar{w}_{1} \partial w}\right|^{2} d u d v .
$$

La forme quadratique - $(2 / \pi)\left(\partial^{2} \lambda\left(w_{1}\right) / \partial w_{1} \partial \bar{w}_{1}\right)\left|d w_{1}\right|^{2}$ sur $R$ est égale à la métrique de Bergmann $K(w)|d w|^{2}$ sur $R$.

Concernant la variation de cette métrique en cas où la surface de Riemann à bord $\mathfrak{S}(z)$ se varie et déforme analytiquement avec paramètre complexe $z \in \Delta$, on aura une propriété intéressante:

CorollaIre 3. Soit $(\mathfrak{D}, \Delta, \pi)$ le triple vérifiant aux conditions $(L),(T)$. Posons $K(z, w)|d w|^{2}$ la métrique de Bergmann sur chaque $\mathfrak{D}(z)$ où $z \in \Delta$. Alors, le coefficient $K(z, w)$ est logarithmiquement pluri-sousharmonique par rapport $\grave{a} z$ dans $\Delta$.

Precisément dit, soit $(z, w) \in \Delta_{0} \times \delta, \pi$-coordonnées quelconques de $\mathfrak{D}, \Delta_{0}$ étant un polydisque de $\Delta$ et $\delta=\{|w|<\tau\}$. Pour $z$ fixé dans $\Delta_{0}$, on pose $K(z, w)|d w|^{2}$ la forme quadratique dans $\delta$ définie par la métrique de Bergmann 
sur $\mathfrak{D}(z)$. Alors $\log K(z, w)$ est, pour $w$ fixé dans $\delta$, pluri-sousharmonique par rapport à $z \in \Delta_{0}$.

DÉmonstration. On la partage trois cas. Cas $1: n=1$ et $(\mathfrak{D}, \Delta, \pi)$ est égale à $(D, \Delta, p)$ tel que $D$ soit un ouvert connexe multivalent étale au-dessus du polycylindre $\Delta \times C, C$ étant le plan $w$, qui satisfait à la condition $(R)$ de $\S 2$. D'après $(R 1), w$ étant la coordonnée de chaque point sur chaque $D(z)$ où $z \in \Delta$, on peut poser la métrique de Bergmann sur $D(z), K(z, w)|d w|^{2}$ sur $D(z)$ de façon que $K(z, w)$ soit fonction uniforme réelle positive dans $D$. En ce cas, on va vérifier $\log K(z, w)$ est, pour tout $w$ fixé, sousharmonique par rapport à $z \in \Delta$. Pour cela, soit $\left(z, w_{1}\right) \in D . \quad g\left(z, w_{1}, w\right)$ désigne la fonction de Green de $D(z)$ avec pôle à $w_{1}$, et $\lambda\left(z, w_{1}\right)$ la constante de Robin de $D(z)$ par rapport au point $w_{1}$ et à la coordonnée $w$. On a le développement dans un voisinage de $\left(z, w_{1}\right)$ dans $D$,

$$
g\left(z, w_{1}, w\right)=\log \frac{1}{\left|w-w_{1}\right|}+\lambda\left(z, w_{1}\right)+\operatorname{Re}\left\{\sum_{n=1}^{\infty} a_{n}\left(z, w_{1}\right)\left(w-w_{1}\right)^{n}\right\}
$$

D'après la formule 2 , on a pour $\left(z, w_{1}\right) \in D$,

$$
\frac{\partial^{2} \lambda\left(z, w_{1}\right)}{\partial z \partial \bar{z}}=-\frac{4}{\pi} \iint_{D(z)}\left|\frac{\partial^{2} g\left(z, w_{1}, w\right)}{\partial \bar{z} \partial w}\right|^{2} d u d v
$$

Par $w_{1} \bar{w}_{1}$-différentielle, on en a

$$
\frac{\partial^{4} \lambda\left(z, w_{1}\right)}{\partial w_{1} \partial \bar{w}_{1} \partial z \partial \bar{z}}=-\frac{4}{\pi} \iint_{D(z)}\left\{\frac{\partial^{2}}{\partial w_{1} \partial \bar{w}_{1}}\left(\left|\frac{\partial^{2} g\left(z, w_{1}, w\right)}{\partial \bar{z} \partial w}\right|^{2}\right)\right\} d u d v
$$

Puisque $g\left(z, w_{1}, w\right)$ est, pour $w$ fixé dans $D(z)$, harmonique par rapport à $w_{1}$ sur $D(z)-\{w\}$, d'où $\partial^{2} g / \partial \bar{z} \partial w$ l'est aussi sur toute surface $D(z)$, on obtient pour $\left(z, w_{1}\right) \in D$

$$
\frac{\partial^{4} \lambda\left(z, w_{1}\right)}{\partial z \partial \bar{z} \partial w_{1} \partial \bar{w}_{1}}=-\frac{4}{\pi} \iint_{D(z)}\left(\left|\frac{\partial^{3} g}{\partial \bar{z} \partial \bar{w}_{1} \partial w}\right|^{2}+\left|\frac{\partial^{3} g}{\partial z \partial \bar{w}_{1} \partial \bar{w}}\right|^{2}\right) d u d v
$$

Soit $z_{0} \in \Delta$. On a pour $w_{1} \in D\left(z_{0}\right)$, par la même méthode que (3.7)

$$
\frac{\partial \lambda}{\partial z}\left(z_{0}, w_{1}\right)=\frac{\sqrt{-1}}{\pi} \int_{C}\left\{\frac{\partial g}{\partial z} \frac{\partial g}{\partial w}\right\}_{\left(z_{0}, w_{1}, w\right)} d w
$$

où $C=\partial D\left(z_{0}\right)$. Il vient pareillement

$$
\frac{\partial^{3} \lambda}{\partial w_{1} \partial \bar{w}_{1} \partial z}\left(z_{0}, w_{1}\right)=\frac{\sqrt{-1}}{\pi} \int_{C}\left\{\frac{\partial^{2} g}{\partial \bar{w}_{1} \partial z} \frac{\partial^{2} g}{\partial w_{1} \partial w}+\frac{\partial^{2} g}{\partial w_{1} \partial z} \frac{\partial^{2} g}{\partial \bar{w}_{1} \partial w}\right\}_{\left(z_{0}, w_{1}, w\right)} d w .
$$

Puisque $g\left(z, w_{1}, w\right)=0$ pour $\left(z, w_{1}\right) \in D$ et $w \in \partial D(z)$, il vient d'après $(R 3)$, 
$g\left(z, w_{1}, \xi_{0}(z, \zeta)\right)=0$ pour $\left(z, w_{1}\right) \in D$ et $\zeta \in C$. Par $w_{1}$-différentielle on y a $g\left(z, w_{1}, \xi_{0}(z, \zeta)\right) / \partial w_{1}=0$, d'où, par $z$-différentielle,

$$
\frac{\partial^{2} g}{\partial z \partial w_{1}}\left(z, w_{1}, \zeta\right)+\frac{\partial^{2} g}{\partial w \partial w_{1}}\left(z, w_{1}, \xi_{0}(z, \zeta)\right) \frac{\partial \xi_{0}}{\partial z}(z, \zeta)=0
$$

pour $\left(z, w_{1}\right) \in D$ et $\zeta \in C$. En particulier, on a d'après $(R 3)$,

$$
\frac{\partial^{2} g}{\partial z \partial w_{1}}\left(z_{0}, w_{1}, \zeta\right)+\frac{\partial^{2} g}{\partial w \partial w_{1}}\left(z_{0}, w_{1}, \zeta\right) \frac{\partial \xi_{0}}{\partial z}\left(z_{0}, \zeta\right)=0 .
$$

Par le pareil procédé pour $\partial g / \partial \bar{w}_{1}$, on a enfin, pour $z=z_{0}, w_{1} \in D\left(z_{0}\right)$ et $\zeta \in C$,

$$
\frac{\partial^{2} g}{\partial \bar{w}_{1} \partial z} \frac{\partial^{2} g}{\partial w_{1} \partial w}=\frac{\partial^{2} g}{\partial w_{1} \partial z} \frac{\partial^{2} g}{\partial \bar{w}_{1} \partial w}=-\frac{\partial \xi_{0}}{\partial z} \frac{\partial^{2} g}{\partial w_{1} \partial w} \frac{\partial^{2} g}{\partial \bar{w}_{1} \partial w} .
$$

Il en résulte que

$$
\frac{\partial^{3} \lambda}{\partial w_{1} \partial \bar{w}_{1} \partial z}\left(z_{0}, w_{1}\right)=\frac{2 \sqrt{-1}}{\pi} \int_{\sigma}\left\{\frac{\partial^{2} g}{\partial w_{1} \partial z} \frac{\partial^{2} g}{\partial \bar{w}_{1} \partial w}\right\}_{\left(z_{0}, w_{1}, w\right)} d w .
$$

Puisque toutes les deux fonctions sous l'intégrale sont, d'après le développement (4.1), régulières sans singularité dans $D\left(z_{0}\right)$, il vient grâce à la formule de Stokes

$$
=\frac{2 \sqrt{-1}}{\pi} \iint_{D\left(z_{0}\right)}\left\{\frac{\partial^{3} g}{\partial \bar{w} \partial w_{1} \partial z} \frac{\partial^{2} g}{\partial \bar{w}_{1} \partial w}\right\}_{\left(z_{0}, w_{1}, w\right)} \overline{d w} \wedge d w .
$$

$z_{0}$ étant quelconque, on a pour $\left(z, w_{1}\right) \in D$

$$
\frac{\partial^{3} \lambda\left(z, w_{1}\right)}{\partial z \partial w_{1} \partial \bar{w}_{1}}=-\frac{4}{\pi} \iint_{D(z)} \frac{\partial^{3} g}{\partial z \partial w_{1} \partial \bar{w}} \frac{\partial^{2} g}{\partial \bar{w}_{1} \partial w} d u d v .
$$

En vertu de l'inégalité de Schwarz, il vient, d'après (4.2) et le corollaire 2, pour $\left(z, w_{1}\right) \in D$

$$
\begin{gathered}
\left|\frac{\partial^{3} \lambda\left(z, w_{1}\right)}{\partial z \partial w_{1} \partial \bar{w}_{1}}\right|^{2} \leqq \frac{16}{\pi^{2}}\left(\iint_{D(z)}\left|\frac{\partial^{3} g}{\partial z \partial w_{1} \partial \bar{w}}\right|^{2} d u d v\right)\left(\iint_{D(z)}\left|\frac{\partial^{2} g}{\partial \bar{w}_{1} \partial w}\right|^{2} d u d v\right) \\
\leqq\left(-\frac{\partial^{4} \lambda\left(z, w_{1}\right)}{\partial z \partial \bar{z} \partial w_{1} \partial \bar{w}_{1}}\right) \cdot\left(-\frac{\partial^{2} \lambda\left(z, w_{1}\right)}{\partial w_{1} \partial \bar{w}_{1}}\right) .
\end{gathered}
$$

D'après $K\left(z, w_{1}\right)=(-2 / \pi) \partial^{2} \lambda\left(z, w_{1}\right) / \partial w_{1} \partial \bar{w}_{1}$ pour $\left(z, w_{1}\right) \in D$, cette inégalité n'est autre que $\partial^{2} \log K(z, w) / \partial z \partial \bar{z} \geqq 0$ pour $(z, w) \in D$, ce qu'on demande.

Cas 2: On traite $n=1$, le triple (D, $\Delta, \pi), \pi$-coordonnées $(z, w) \in \Delta_{0} \times \delta$ de D et la forme $K(z, w)|d w|^{2}$ sur $\delta$ mentionnés dans le colloraire 3 . Soit $\left(z_{0}, w_{0}\right)$ $\in \Delta_{0} \times \delta$. On dénote $\alpha\left(\Delta_{0}\right)$ l'ensemble analytique dans $\mathscr{D} \mid \Delta_{0}$ correspondant à 
la droite définie par $w=w_{0}$ dans $\Delta_{0} \times \delta$. D'après $\S 2$, il existe un disque $\Delta_{0}^{\prime}$ de centre $z_{0}$ dans $\Delta_{0}$ et un ouvert connexe $D(\subset U)$ étalé au-dessus du dicylindre $\Delta_{0}^{\prime} \times C$ où $C$ est le plan $W$ tels que $\left(\mathfrak{D}\left|\Delta_{0}^{\prime}, \Delta_{0}^{\prime}, \pi\right| \Delta_{0}^{\prime}\right)$ soit équivalent à $\left(D, \Delta_{0}^{\prime}, p\right)$, $p$ la première projection, satisfaisant aux conditions $(R) \operatorname{de} \S 2$ et $(R 4)$ de $\S 3$. Il existe donc une transformation analytique: $(z, w) \rightarrow\left(z, W_{1}(z, w)\right)$ de $\Delta_{0}^{\prime} \times \delta$ dans $D$. D'après $(R 4)$, on a $W_{1}\left(z, w_{0}\right)=0$ pour $z \in \Delta_{0}^{\prime}$, d'où $\left(\partial W_{1} / \partial z\right)\left(z_{0}, w_{0}\right)=0$. Pour $\left(z, W_{1}\right) \in D, G\left(z, W_{1}, W\right)$ (resp. $\Lambda\left(z, W_{1}\right)$ ) désigne la fonction de Green de $D(z)$ avec pôle à $W_{1}$ (resp. la constante de Robin de $D(z)$ par rapport à $W_{1}$ et à la coordonnée $W$ ). D'après (3.1), il vient, pour tout $z$ fixé dans $\Delta_{0}^{\prime}$,

$$
\lambda\left(z, w_{1}\right)=\Lambda\left(z, W_{1}\left(z, w_{1}\right)\right)-\log \left|\frac{\partial W_{1}}{\partial w_{1}}\left(z, w_{1}\right)\right|
$$

pour $w_{1} \in \delta$. Puisque $W_{1}\left(z, w_{1}\right)$ est analytique pour $\left(z, w_{1}\right) \in \Delta_{0}^{\prime} \times \delta$, il vient

$$
\frac{\partial^{2} \lambda}{\partial w_{1} \partial \bar{w}_{1}}\left(z, w_{1}\right)=\frac{\partial^{2} \Lambda}{\partial W_{1} \partial \bar{W}_{1}}\left(z, W_{1}\left(z, w_{1}\right)\right) \cdot\left|\frac{\partial W_{1}}{\partial w_{1}}\left(z, w_{1}\right)\right|^{2} .
$$

Il en résulte pour $\left(z, w_{1}\right) \in \Delta_{0}^{\prime} \times \delta$

$$
\begin{aligned}
\frac{\partial^{2}}{\partial z \partial \bar{z}} & \log \left(-\frac{\partial^{2} \lambda}{\partial w_{1} \partial \bar{w}_{1}}\left(z, w_{1}\right)\right)=\frac{\partial^{2}}{\partial z \partial \bar{z}} \log \left(-\frac{\partial^{2} \Lambda}{\partial W_{1} \partial \bar{W}_{1}}\left(z, W_{1}\left(z, w_{1}\right)\right)\right) \\
= & \left\{\frac{\partial^{2}}{\partial z \partial \bar{z}} \log \left(-\frac{\partial^{2} \Lambda}{\partial W_{1} \partial W_{1}}\right)\right\}_{\left(z, W_{1}\left(z, w_{1}\right)\right)} \\
& \left.+\mid \frac{\partial W_{1}\left(z, w_{1}\right)}{\partial z}\right]^{2} \cdot\left\{\frac{\partial^{2}}{\partial W_{1} \partial W_{1}} \log \left(-\frac{\partial^{2} \Lambda}{\partial W_{1} \partial W_{1}}\right)\right\}_{\left(z, W_{1}\left(z, w_{1}\right)\right)} \\
& +2 \operatorname{Re}\left[\frac{\partial W_{1}\left(z, w_{1}\right)}{\partial z}\left\{\frac{\partial^{2}}{\partial \bar{z} \partial W_{1}} \log \left(-\frac{\partial^{2} \Lambda}{\partial W_{1} \partial W_{1}}\right)\right\}_{\left(z, W_{1}\left(z, w_{1}\right)\right)}\right] .
\end{aligned}
$$

D’après $\left(\partial W_{1} / \partial z\right)\left(z_{0}, w_{0}\right)=0$, on a

$$
\left\{\frac{\partial^{2}}{\partial z \partial \bar{z}} \log \left(-\frac{\partial^{2} \lambda}{\partial w_{1} \partial \bar{w}_{1}}\right)\right\}_{\left(z_{0}, w_{0}\right)}=\left\{\frac{\partial^{2}}{\partial z \partial \bar{z}} \log \left(-\frac{\partial^{2} \Lambda}{\partial W_{1} \partial W_{1}}\right)\right\}_{\left(z_{0}, 0\right)} \cdot
$$

Grâce au cas 1 , le second membre est non-négative. $\left(z_{0}, w_{0}\right)$ étant quelconque de $\Lambda_{0} \times \delta$, on trouve que $\partial^{2} \log K(z, w) / \partial z \partial \bar{z} \geqq 0$ pour $(z, w) \in \Delta_{0} \times \delta$.

Cas 3. On traite $n \geqq 2$. Soit $z_{i}=z_{0 i}+a_{i} t(i=1, \cdots, n)$ la droite quelconque $L$ passant par $z_{0}=\left(z_{01}, \cdots, z_{0 n}\right)$ dans $\Delta_{0}$, où $t$ se varie dans un disque $\Delta^{*}\left(=\left\{|t|<\rho^{*}\right\}\right)$ du plan $t$. Quand on pose $\mathfrak{D}^{*}=\pi^{-1}(L)$ et $\pi^{*}=$ la restriction de $\pi \operatorname{sur} \mathfrak{D}^{*}$, alors $\left(\mathfrak{D}^{*}, \Delta^{*}, \pi^{*}\right)$ est le triple satisfaisant aux conditions $(L),(T)$ et $n=1$. Il en résulte d'après le cas 2 que $\partial^{2} \log K\left(\left(z_{0 i}+a_{i} t\right), w\right) / \partial t \partial \bar{t} \geqq 0$ pour $(t, w) \in \Delta^{*} \times \delta . \quad \log K(z, w)$ est donc, pour $w$ fixé dans $\delta$, pluri-sousharmonique par rapport à $z \in \Delta_{0}$. Le corollaire 3 est démontré. 
On peut aller un peu plus loin. Supposons que $(\mathscr{D}, \Delta, \pi)$ satisfasse aux conditions $(L),(T)$. Soit $P_{0} \in \mathfrak{D}$ tel que $\pi\left(P_{0}\right)=z_{0}$, et $(z, w) \in \Delta_{0} \times \delta$ où $\delta=$ $\{|w|<\tau\} \pi$-coordonnées d'un voisinage $\mathscr{V}$ de $P_{0}$ dans $\mathfrak{D}$. On écrit $P(z, w)$ le point de $\mathscr{V}$ correspondant à $(z, w)$ de $\Delta_{0} \times \delta$. Pour tout $\left(z_{1}, w_{1}\right)$ fixé dans $\Delta_{0} \times$ $\delta$, on considère la constante de Robin $\lambda\left(z_{1}, w_{1}\right)$ de la fibre $\mathscr{D}\left(z_{1}\right)$ par rapport au point $P\left(z_{1}, w_{1}\right)$ et à la coordonnée locale $\delta$. On a ainsi obtenu une fonction réelle $\lambda(z, w)$ défine dans $\Delta_{0} \times \delta$. D'après les corollaires 1,2 , il vient évidemment la

REMARQUE 5. $\lambda(z, w)$ est fonction pluri-surharmonique par rapport à $(z, w)$ dans $\Delta_{0} \times \delta$.

Soit $P_{1} \in \mathscr{D}$ et $(z, W) \in \Delta_{1} \times \delta_{1}$ des $\pi$-coordonées d'un voisinage $\mathscr{V}_{1}$ de $P_{1}$ dans $\mathfrak{D}$ tel que $\mathscr{V} \cap \mathscr{V}_{1} \neq \phi$. Comme $\lambda(z, w)$ définie dans $\Delta_{0} \times \delta$, on forme la fonction réelle $\Lambda(z, W)$ pour $(z, W) \in \Delta_{1} \times \delta_{1}$. Il existe une transformation analytique d'une partie de $\Delta_{0} \times \delta$ sur celle de $\Delta_{1} \times \delta_{1}$ de la forme $T:(z, w) \rightarrow$ $(z, W(z, w))$. On a d'après $(3.1)$

$$
\lambda(z, w)=\Lambda \circ T(z, w)-\log \left|\frac{\partial W(z, w)}{\partial w}\right| .
$$

Il s'ensuit que pour $z=\left(z_{1}, \cdots, z_{n}\right)$ et $i, j=1, \cdots, n$,

$$
\frac{\partial^{2} \lambda}{\partial z_{i} \partial \bar{z}_{j}}=\frac{\partial^{2} \Lambda \circ T}{\partial z_{i} \partial \bar{z}_{j}} ; \frac{\partial^{2} \lambda}{\partial z_{i} \partial \bar{w}}=\frac{\partial^{2} \Lambda \circ T}{\partial z_{i} \partial \bar{w}} ; \frac{\partial^{2} \lambda}{\partial w \partial \bar{w}}=\frac{\partial^{2} \Lambda \circ T}{\partial w \partial \bar{w}} .
$$

C'est-à-dire, la donnée, aux chaques $\pi$-coordonnées $(z, w)=\left(z_{1}, \cdots, z_{n}, w\right) \in \Delta_{0}$ $\times \delta$ de $\mathfrak{D}$, de la forme quadratique $\sum_{i, j=1}^{n+1}\left(-\partial^{2} \lambda / \partial z_{i} \partial \bar{z}_{j}\right)\left(z_{1}, \cdots, z_{n}, z_{n+1}\right) d z_{i} d \bar{z}_{j}$ où $z_{n+1}=w$ définit une pseudo-métrique $d s_{\varpi}^{2}$ sur tout $\mathfrak{D}$. On résume le

THÉORÈme 1. Soit $M$ une variété de dimension complexe $n$. Soit donné un triple $\left(\mathscr{D}_{k}, \Delta_{k}, \pi_{k}\right)$ satisfaisant aux conditions $(L),(T)$ pour chaques coordonnées locales $\Delta_{k}(k=1,2, \cdots)$ de $M$ de façon que $\mathfrak{\wp}=\left\{\left(\mathfrak{D}_{k}, \Delta_{k}, \pi_{k}\right): k=1,2, \cdots\right\}$ soit famille holomorphe sur $M$. Alors la donnée, à chaque $\mathfrak{D}_{k}(k=1,2, \ldots)$ de la pseudo-métrique ci-dessus $d s_{\Phi_{k}}^{2}$ détermine une pseudo-métrique kaelérienne $d s^{2}$ $=\sum_{i, j=1}^{n+1} g_{i \grave{j}} d z_{i} \overline{d z}_{j}$ où $z_{n+1}=w$ sur toute $\widetilde{\lessgtr}$ ayant les propriétés: (1) $g_{n+1} \overline{n+1}(z, w)$ est positive et pour $w$ fixé, logarithmiquement pluri-soushamonique par rapport $\grave{a} z$; (2) pour toute suite de point $P_{\nu}$ de $\widetilde{\mho}$ sans point d'accumuration de $\widetilde{\mho}$ telle que la suite de la projection de $P_{\nu}$ sur $M$ converge vers un point $z_{0}$ de $M$, la distance $d\left(O, P_{\nu}\right)$ d'un point $O$ de $\widetilde{\jmath}$ tend vers l'infini.

Preuve. D'après l'expression (4.5) et le corollaire 3 , il reste de prouver la propriété (2). Il suffit de la prouver sous la condition que tout $P_{\nu}$ et $O$ soient contenus dans un triple $(\mathfrak{D}, \Delta, \pi)$ de $\widetilde{\gamma}$ tel que $\pi\left(P_{\nu}\right) \rightarrow z_{0} \in \Delta$ et $P_{\nu} \rightarrow \partial \mathscr{D}\left(z_{0}\right)$ 
pour $\nu \rightarrow \infty$. Soit $P_{0} \in \partial \mathfrak{S}\left(z_{0}\right)$. D'après la remarque 2 de $\S 2$, on trouve $\pi$ coordonnées $\Delta_{0} \times \delta$ où $\delta=\{|\boldsymbol{w}|<1\}$ d'un voisinage $\mathscr{V}$ de $P_{0}$ dans $\hat{\mathfrak{D}}$ telles que $P_{0}$ corresponde à $\left(z_{0}, 0\right)$ et $\mathscr{V}_{P_{0}} \cap \mathfrak{D}$ corresponde à l'ensemble produit $\Delta_{0} \times \delta_{+}$ où $\delta_{+}=\{w \in \delta: \operatorname{Im} w>0\}$. Pour $z \in \Delta_{0}, g\left(z, w_{1}, w\right)$ (resp. $\lambda\left(z, w_{1}\right)$ ) désigne la fonction de Green de $\mathfrak{S}(z)$ avec pôle au point $w_{1}$ de $\delta_{+}$(resp. la constante de Robin de $\mathfrak{D}(z)$ par rapport à $\boldsymbol{w}_{1}$ et à $\left.\delta_{+}\right)$. Il est évident que $g\left(z, w_{1}, w\right)$ $\log \left|\left(\boldsymbol{w}-\overline{\boldsymbol{w}}_{1}\right) /\left(\boldsymbol{w}-\boldsymbol{w}_{1}\right)\right|$ est analytique au sens réel pour $\left(z, \boldsymbol{w}_{1}, w\right) \in \Delta_{0} \times \delta \times \delta$, d'où que $\lambda\left(z, w_{1}\right)-\log 2\left(\operatorname{Im} w_{1}\right)$ est analytique au sens réel pour $\left(z, w_{1}\right) \in \Delta_{0} \times \delta$. Il s'ensuit que pour $\left(z, w_{1}\right) \in \Delta_{0} \times \delta_{+}$

$$
d s_{Ð}^{2}\left(z, w_{1}\right)=\frac{4\left|d w_{1}\right|^{2}}{\left(\operatorname{Im} w_{1}\right)^{2}}+d s_{0}^{2}\left(z, w_{1}\right)
$$

telle que tous les coefficients de $d s_{0}^{2}\left(z, w_{1}\right)$ soient bornés dans $\Delta_{0} \times \delta_{+} \cdot P_{0}$ étant quelconque de $\partial \mathfrak{D}\left(z_{0}\right), \partial \mathfrak{D}\left(z_{0}\right)$ est recouverte par un nombre fini de tels $\mathscr{V}$ dans Dิ avec $\pi$-coordonnées $(z, w) \in \Delta_{0} \times \delta$ tels qu'on y ait l'expression (4.6) de la pseudo-métrique $d s_{D}^{2}$. On en aisément $d\left(O, P_{\nu}\right) \rightarrow+\infty$ pour $P_{\nu} \rightarrow \partial \supseteqq\left(z_{0}\right)$. Le théorème est prouvé.

Soit (D) $\Delta, \pi)$ un triple satisfaisant aux conditions $(L),(T)$ avec la pseudométrique ci-dessus $d s_{\circledast}^{2}$. Soit $\alpha$ une section analytique de $\mathfrak{D} \operatorname{sur} \Delta . \quad d s_{\circledast}^{2}$ de $\mathfrak{D}$ induit canoniquement la pseudo-métrique $d s_{\triangleright}^{2} \mid \alpha(\Delta)$ de la surface analytique $\alpha(\Delta)$ de $\mathfrak{D}$. Par définition, $d s_{\triangleright}^{2} \mid \alpha(\Delta)$ est identiquement nulle sur $\alpha(\Delta)$ si et seulement si la fonction $\lambda(z, \alpha(z))$ est pluri-harmonique pour $z \in \Delta$. Par exemple, quand $(\mathfrak{D}, \Delta, \pi)$ est équivalent à celui trivial, on trouve, d'après la remarque 3 , une famille de sections analytiques $\left\{\alpha_{t}\right\}_{t}$ de $\mathscr{D}$ telles que chaque $d s_{\circledast}^{2} \mid \alpha_{t}(\Delta)$ soit nulle sur $\alpha_{t}(\Delta)$. On va envisager le problème réciproque ${ }^{(\#)}$. Pour simplicité d'écriture, on donne une

DÉFInITIon. Soit $\mathfrak{D}$ un ouvert connexe étalé au-dessus de l'espace produit $\Delta \times R, R$ étant une surface de Riemann. Soit $\beta$ un ensemble analytique de dimension $n$ de $\mathfrak{D}$ tel que la projection de $\beta$ sur $\Delta \times R$ soit de la forme $\{(z, \beta(z)) \in \Delta \times R: z \in \Delta\}$. Si $\beta(z)$ est constante (resp. non-constante) comme fonction de $z \in \Delta$, l'ensemble $\beta$ de $\mathfrak{D}$ est dit constante (resp. non-constante) pour $z \in \Delta$.

On commence un lemme concernant le prolongement analytique.

LEMME 2. Supposons que $f\left(z, w_{1}\right)$ soit fonction méromorphe définie dans un ouvert connexe $D_{1}$ étalé au-dessus du polycylindre $\Delta \times \boldsymbol{P}^{1}, \Delta$ étant un polydisque de $\boldsymbol{C}^{n}, \boldsymbol{P}^{1}$ la sphère de Riemann $\left\{\left|w_{1}\right| \leqq \infty\right\}$, satisfaisant à la condition suivante: il existe un sous-ensemble de $D_{1}$ de la forme $\Delta \times l_{1}, l_{1}$ étant un arc

(\#) M. Akira Takeuchi m'a suggeré de traiter cette réciproque. 
dans $\boldsymbol{P}^{1}$ tel que $\Delta \times l_{1}$ se transforme par $T_{f}: z=z, w_{2}=f\left(z, w_{1}\right)$, sur celui de même forme $\Delta \times l_{2}, l_{2}$ étant un arc dans la sphère de Riemann $\boldsymbol{P}^{1}\left(w_{2}\right)$. Quand on désigne par $D_{2}$ l'ouvert connexe étale au-dessus de $\Delta \times \boldsymbol{P}^{1}\left(w_{2}\right)$, de l'image de $D_{1}$ par $T_{f}$, c'est-à-dire, $D_{1}$ et $D_{2}$ sont bijectifs par $T_{f}$, alors (1) dans un voisinage univalent $\Delta_{0} \times \delta$ du point ordinaire quelconque $\left(z_{0}, w_{01}\right)$ de $D_{1}, f\left(z, w_{1}\right)$ ne dépend pas de $z \in \Delta_{0}$; (2) chaque composante irréductible $\beta_{1}$ non-constante pour $z \in \Delta$ de surfaces de ramification de $D_{1}$, se transforme par $T_{f}$ en celle $\beta_{2}$ non-constante pour $z \in \Delta$ de $D_{2}$; (3) l'ordre de ramification de $D_{1}$ le long de telle $\beta_{1}$ est égale à celui de $D_{2}$ le long de $\beta_{2}$, soit $m-1(\geqq 1)$; (4) les $m$ points de $D_{1}$ voisins de $\beta_{1} q u i$ se trouve au-dessus d'un point ordinaire $\left(z, w_{01}\right) \in \Delta \times \boldsymbol{P}^{1}$ se transforme par $T_{f}$ en les $m$ points de $D_{2}$ voisins de $\beta_{2}$ se trouvant au-dessus du point $\left(z, w_{02}\right) \in \Delta \times$ $\boldsymbol{P}^{1}\left(w_{2}\right)$ tel que $w_{02}$ dépende de $w_{01}$, mais non de $z \in \Delta$.

PReuve. Soit $z_{0} \in \Delta, \zeta_{01} \in l_{1}$ et $\zeta_{02}=f\left(z_{0}, \zeta_{01}\right) \in l_{2}$ tels que $\left(z_{0}, \zeta_{01}\right)\left(\operatorname{resp} .\left(z_{0}, \zeta_{02}\right)\right)$ le point ordinaire de $D_{1}$ (resp. $D_{2}$ ). On prend un disque $\gamma_{i}(i=1,2)$ de centre $\zeta_{0 i}$ tel que $\gamma_{i}$ se divise en deux parties $\left\{\gamma_{i}^{(1)}, \gamma_{i}^{(2)}\right\}$ par l'arc $l_{i}$ telle que $\Delta \times \gamma_{1}^{(j)}$ $(j=1,2)$ corresponde à une partie de $\Delta \times \gamma_{2}^{(j)}$ par $T_{f}$. On forme une transformation conforme $W_{i}=G_{i}\left(w_{i}\right)$ de $\gamma_{i}^{(1)}$ sur la partie $C_{i}^{+}$du plan $W_{i}$ qui est audessus de l'axe réel tel que $G_{i}\left(\zeta_{0 i}\right)=0$. On pose pour $\left(z, W_{1}\right) \in \Delta \times C_{1}^{+}, W_{2}=$ $\tilde{f}\left(z, W_{1}\right)=G_{2}\left(f\left(z, G_{1}^{-1}\left(W_{1}\right)\right)\right)$. D'après la condition, chaque $\tilde{f}\left(z, W_{1}\right)$ où $z \in \Delta$ transforme conformément $C_{1}^{+}$dans $C_{2}^{+}$de façon que $\tilde{f}\left(z, C_{1}^{+} \cap\{1\right.$ 'axe réel $\left.\}\right) \subset C_{2}^{+}$ $\cap\{$ l'axe réel $\}$. Donc, chaque $\tilde{f}\left(z, W_{1}\right)$ où $z \in \Delta$ se prolonge analytiquement pour $W_{1}$ dans un voisinage de 0 et se développe: $\tilde{f}\left(z, W_{1}\right)=\sum_{n=0}^{\infty} a_{n}(z) W_{1}^{n}$ telle que $a_{n}(z)(n=0,1, \cdots)$ soit réel. En autre côté, puisque $\tilde{f}\left(z, W_{1}\right)$ est analytique pour $\left(z, W_{1}\right) \in \Delta \times C_{1}^{+}$, tout $a_{n}(z)$ est analytique par rapport à $z \in \Delta$, d'où $a_{n}(z)$ devient une constante dans $\Delta$. Il s'ensuit que $f\left(z, w_{1}\right)$ ne dépend pas de $z \in \Delta$. On a

$$
\frac{\partial f}{\partial z_{i}}\left(z, w_{1}\right)=0(i=1, \cdots, n) \quad \text { pour }\left(z, w_{1}\right) \in \Delta \times \gamma_{1}
$$

On trace une courbe $\Gamma_{1}$ sur $D_{1}\left(z_{0}\right)$ partant de $\zeta_{01}$ et arrivant à $w_{01}$ de (1) de façon que $\Gamma_{1}$ (resp. $\Gamma_{2}=f\left(z_{0}, \Gamma_{1}\right)$ ) n'intersecte pas de point de ramification de $D_{1}\left(z_{0}\right)$ (resp. $D_{2}\left(z_{0}\right)$ ). D'après le prolongement analytique de $f\left(z, w_{1}\right)$ le long de $\Gamma_{1}$, il existe, d'après (4.7), un voisinage $\Delta_{0} \times \delta$ de $\left(z_{0}, w_{01}\right)$ dans $D_{1}$ tel que $\partial f / \partial z_{i}(z, w)$ soit nulle pour $i=1, \cdots, n$ et $(z, w) \in \Delta_{0} \times \delta$, d'où on conclut (1). La composante $\beta_{1}$ de (2) se trouve au-dessus de l'ensemble $\left\{\left(z, \beta_{1}(z)\right): z \in \Delta\right\}$ de $\Delta \times \boldsymbol{P}^{1}$, et par l'hypothèse de (2), il existe $(1 \leqq) j(\leqq n)$ tel que $\partial \beta_{1}(z) / \partial z_{j} \neq 0$ dans $\Delta$. Soit $m_{1}-1(\geqq 1)$ l'ordre de ramification de $D_{1}$ pour $\beta_{1}$. Soit $\beta_{2}=T_{f} \circ \beta_{1}$ se trouvant au-dessus de l'ensemble $\left\{\left(z, \beta_{2}(z)\right): z \in \Delta\right\}$ de $\Delta \times \boldsymbol{P}^{1}\left(w_{2}\right)$, et soit $m_{2}-1(\geqq 0)$ l'ordre de ramification de $D_{2}$ pour $\beta_{2}$. Il vient, par $\beta_{2}(z)=f\left(z, \beta_{1}(z)\right)$, dans un voisinage $\mathscr{V}$ de $\beta_{1}$, 


$$
f\left(z, w_{1}\right)=\beta_{2}(z)+b_{1}(z)\left(w_{1}-\beta_{1}(z)\right)^{m_{2} / m_{1}}+b_{2}(z)\left(w_{1}-\beta(z)\right)^{\left(m_{2}+1\right) / m_{1}}+\cdots
$$

telle que $b_{1}(z) \neq 0$ et $b_{n}(z)(n=1,2, \cdots)$ est analytique et uniforme pour $z \in \Delta$. Par $z_{j}$-différentielle et (4.7), on a pour $\left(z, w_{1}\right) \in \mathscr{V}$

$$
\begin{aligned}
0=\frac{\partial \beta_{2}(z)}{\partial z_{j}}+\left\{\frac{\partial b_{1}(z)}{\partial z_{j}}(\right. & \left.w_{1}-\beta_{1}(z)\right)^{m_{2} / m_{1}} \\
& \left.+b_{1}(z) \frac{m_{2}}{m_{1}}\left(-\frac{\partial \beta_{1}(z)}{\partial z_{j}}\left(w_{1}-\beta_{1}(z)\right)^{\left(m_{2} / m_{1}\right)-1}\right)\right\}+\cdots
\end{aligned}
$$

Supposons qu'il existe le coefficient $b_{p+1-m_{2}}(z)$ de $\left(w_{1}-\beta_{1}(z)\right)^{p / m_{1}}$ tel que $p$ ne se divise pas par $m_{1}$ et $b_{p+1-m_{2}}(z) \neq 0$ dans $\Delta$. Soit $p_{0}$ le minimum entre tels $p$. Puisqu'il existe dans le second membre de (4.8) un seul terme de la puissance $\left(w_{1}-\beta_{1}(z)\right)^{\left(p_{0} / m_{1}\right)-1}$ dont le coefficient est $-\left(p_{0} / m_{1}\right) b_{p_{0+1-m_{2}}}(z) \partial \beta_{1}(z) / \partial z_{j}$, il est nul pour $z \in \Delta$. Par $\partial \beta_{1}(z) / \partial z_{j} \neq 0$ dans $\Delta$, on a $-b_{p_{0+1-m_{2}}}(z) \equiv 0$ dans $\Delta$, qui est contradiction. Si l'on considère l'inverse, il résulte par $b_{1}(z) \neq 0$ que $m_{1}=m_{2}(=m)$ et pour $(z, w) \in \mathscr{V}$

$$
f\left(z, w_{1}\right)=\beta_{2}(z)+\sum_{n=1}^{\infty} c_{n}(z)\left(w_{1}-\beta_{1}(z)\right)^{n}
$$

telle que $c_{1}(z)=b_{1}(z)$ et $c(z)(n=1,2, \cdots)$ soient fonctions analytiques uniformes pour $z \in \Delta$, d'où (3). D'après (4.8) on obtient $\partial \beta_{2}(z) / \partial z_{j} \neq 0$ dans $\Delta$, d'où (2). L'expression de $f(z, w)$ plus haute entraîne (4). Le lemme 2 est prouvé.

On prépare ensuite un lemme, lui même étant intéressant.

LEMME 3. Soit $(\mathfrak{S}, \Delta, \pi)$ un triple vérifiant aux conditions $(L),(T)$. Supposons que $(\mathfrak{D}, \Delta, \pi)$ ne soit pas équivalent à celui trivial et qu'il existe $\mu$ sections analytiques $\alpha_{k}(k=1, \cdots, \mu)$ de D sur $\Delta$ satisfaisant aux conditions suivantes (i) $\mu \geqq 2$; (ii) la constante de Robin $\lambda_{\alpha_{k}}(z)$ de $\mathfrak{S}(z)$ par rapport au point $\alpha_{k}(z)$ soit pluri-harmonique pour $z \in \Delta$. Alors il existe une surface de Riemann $R$ à bord telle que (I), $\Delta, \pi)$ soit équivalent à un triple $(\boldsymbol{D}, \Delta, \boldsymbol{p})$ où $\boldsymbol{D}$ est un ouvert connexe étalé au-dessus de l'espace produit $\Delta \times R$ et $p$ la première projection, qui a les propriétés suivantes: (1) il n'existe aucun point frontière de $D$ relatif $\grave{a} \Delta \times R$, soit donc $m(\geqq 2)$ la multiplicité de $D$ au-dessus de $\Delta \times R$; (2) chaque section analytique $\alpha_{k}(k=1, \cdots, \mu)$ corresponde à une composante irréductible $\beta_{k}$ constante pour $z \in \Delta$ de surfaces de ramifiction de $D$ telle que l'ordre de ramification de $D$ pour $\beta_{k}$ soit juste $m-1(\geqq 1)$; (3) il existe au moins une composante $s$ non-constante pour $z \in \Delta$ de surface de ramifiction de $D$. La réciproque est évidemment vraie.

DÉmonstration. $1^{\text {ère }}$ étape. Soit $z_{0} \in \Delta$. On a formé dans $\S 2$ le triple $\left(D, \Delta_{0}, p\right)$ équivalent à $\left(\mathfrak{D}\left|\Delta_{0}, \Delta_{0}, \pi\right| \Delta_{0}\right)$ satisfaisant à la condition $(R)$, où $D$ 
( $\subset U$ ) est un ouvert connexe étalé au-dessus de $\Delta_{0} \times C, \Delta_{0}$ étant un polydisque de centre $z_{0}$ dans $\Delta$ et $p$ la première projection. On désigne simplement par $\lambda_{k}(z)$ (resp. $\left.g_{k}(z, w)\right), \lambda_{\alpha_{k}}(z)$ (resp. $\left.g_{\alpha_{k}}(z, w)\right)$ de la formule 2 . Il en résulte d'après la condition (ii) et la remarque 3 de $\S 4$ que chaque $\partial g_{k} / \partial w(k=1, \cdots, \mu)$ est fonction méromorphe et uniforme pour $(z, w) \in D$ avec seul pôle d'ordre un à résidu -1 le long de la surface analytique $\alpha_{k}$ de $D$ (correspondante à celle $\alpha_{k}\left(\Delta_{0}\right)$ de $\left.\mathfrak{D} \mid \Delta_{0}\right)$. Quand on prend, si nécessaire, plus petit polydisque $\Delta_{0}$ de centre $z_{0}$, il existe un voisinage $V$ de la fibre $D\left(z_{0}\right)$ dans $U$ de la forme $V=$ $\Delta_{0} \times V\left(z_{0}\right)$ où $V\left(z_{0}\right)$ est un ouvert connexe multivalent étalé au-dessus du plan $w$ sans point de ramification tel que $V\left(z_{0}\right) \supset V(z)$ pour tout $z \in \Delta_{0}$ et que $\partial g_{k} / \partial w$ soit méromorphe et uniforme dans $V$. D'après (ii) que $\mu \geqq 2$, on forme la fonction suivante définie pour $(z, w) \in V$ :

$$
W=f(z, w)=\left(\frac{\partial g_{2}}{\partial w} / \frac{\partial g_{1}}{\partial w}\right)(z, w) .
$$

$f(z, w)$ est méromorphe et uniforme dans $V$. D’après $g_{1}(z, w)=g_{2}(z, w)=0$ sur $\partial D(\subset V)$, il vient

$$
\operatorname{Im} f(z, w)=0 \quad \text { sur } \partial D .
$$

Soit fixé $\zeta \in \partial D\left(z_{0}\right)$. Puisque $f\left(z, \xi_{0}(z, \zeta)\right)\left(\xi_{0}(z, \zeta)(\in \partial D(z))\right.$ étant définie en la condition (R3) de $\S 2$ ) est méromorphe pour $z \in \Delta_{0}$, il s'ensuit par (4.9'), $(R 3)$ que $f\left(z, \xi_{0}(z, \zeta)\right)$ est constante $\left(=f\left(z_{0}, \zeta\right)\right)$ pour $z \in \Delta_{0}$. On en a, d'après $(R 3)$, pour tout $z \in \Delta, f(z, \partial D(z))=f\left(z_{0}, \partial D\left(z_{0}\right)\right)$. Quand on pose $D$ (resp. $V$ ) l'image de $D$ (resp. $V$ ) par la transformation analytique $T_{f}:(z, w) \rightarrow(z, f(z, w))$, il s'ensuit que $\boldsymbol{D} \subset \boldsymbol{V}$ et $\boldsymbol{D}$ est un ouvert connexe en nombre fini étalé au-dessus de $\Delta_{0} \times \boldsymbol{P}^{1}(W), \boldsymbol{P}^{1}(W)$ étant la sphère de Riemann $\{|W| \leqq \infty\}$, tel que la frontière $\partial \boldsymbol{D}$ de $\boldsymbol{D}$ dans $\boldsymbol{V}$ s'écrive de la forme

$$
\partial D=\Delta_{0} \times \partial D\left(z_{0}\right)
$$

où $\partial D\left(z_{0}\right)=f\left(z_{0}, \partial D\left(z_{0}\right)\right)$. On sait que le triple $\left(D, \Delta_{0}, p\right)$ est équivalent à tel $\left(D, \Delta_{0}, \tilde{p}\right), \tilde{p}$ étant la projection de $D$ sur $\Delta_{0}$. On écrit, pour $k=1, \cdots, \mu, A_{k}$ $=T_{f} \circ \alpha_{k}$ et $l_{k}-1(\geqq 0)$ l'ordre de ramification de $D$ au-dessus de $\Delta_{0} \times P^{1}(W)$ le long de $A_{k}$.

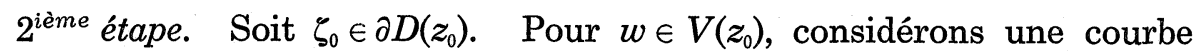
quelconque ne pas nécessairement de Jordan $l\left(z_{0}, w\right)$ sur $V\left(z_{0}\right)$ partant de $\zeta_{0}$ et arrivant à $w$. Soit $l(z, w)$ celle consistant en le segment $\left[\xi_{0}\left(z, \zeta_{0}\right), \zeta_{0}\right]$ et en $l\left(z_{0}, w\right)$. On forme l'intégrale $I_{k}(z, w)(k=1, \cdots, \mu)$ le long de $l(z, w)$, et la fonction $f_{k}$ :

$$
I_{k}(z, w)=2 \int_{\xi_{0}\left(z, \zeta_{0}\right)}^{w} \frac{\partial g_{k}(z, w)}{\partial w} d w ; \quad w_{k}=f_{k}(z, w)=e^{-I_{k}(z, w)}
$$


telle que $\operatorname{Re}\left\{I_{k}(z, w)\right\}=g_{k}(z, w)$ dans $D$. On considère le revêtement $\tilde{V}=\Delta_{0} \times$ $\widetilde{V\left(z_{0}\right)}$ de $V$ tel que $\widetilde{V\left(z_{0}\right)}$ soit le revêtement universel de $V\left(z_{0}\right)$. On écrit désormais pour $K \subset V\left(z_{0}\right)$ (resp. $\left.U \subset V\right), \tilde{K}$ (resp. $\tilde{U}$ ) l'union des sous-ensembles de $\widetilde{V\left(z_{0}\right)}($ resp. $\tilde{V})$ étale au-dessus de $K($ resp. $U)$. On en a $\tilde{U}(z)=\widetilde{U(z)}$ pour $z \epsilon$ $\Delta_{0}$. Alors $f_{k}(z, w)$ est fonction holomorphe et uniforme pour $(z, \tilde{w}) \in \tilde{V}$ ayant les propriétés suivantes: ( $g 1) f_{k}\left(z, \xi_{0}\left(z, \zeta_{0}\right)\right)=1$ pour $z \in \Delta_{0}$; (g2) $f_{k}$ est nulle seulement sur $\tilde{\alpha}_{k} ;(g 3)\left|f_{k}\right|=1$ (resp. $<1$, resp. $\left.>1\right)$ sur $\partial \tilde{D}$ (resp. $\tilde{D}$, resp. $\tilde{V}-$ $\tilde{D})$. On dénote par $D_{k}$ (resp. $\left.V_{k}\right)$ l'image de $\tilde{D}$ (resp. $\left.\tilde{V}\right)$ par la transformation analytique $T_{k}:(z, \tilde{w}) \rightarrow\left(z, f_{k}(z, \tilde{w})\right)$. Il en vient que $D_{k} \subset V_{k}$ et $D_{k}$ est un ouvert connexe en nombre infini étalé au-dessus du polydisque $\Delta_{0} \times U_{k}$ sans point frontière relatif, $U_{k}$ étant le disque unité dans le plan $w$. Puisque la transformation bijective $T_{f}:(z, w) \rightarrow(z, f(z, w))$ de la $1^{\text {ère }}$ étape de $V$ sur $V$ conduit celle $\tilde{T}_{f}:(z, \tilde{w}) \rightarrow(z, f(z, \tilde{w}))$ de $\tilde{V}$ sur $\tilde{V}$ telle que $f(z, \tilde{w})=f(z, w)$ pour $(z, w) \in V$, on a une transformation $S_{k}=T_{k} \circ \tilde{T}_{f}^{-1}$ de $\tilde{V}$ sur $V_{k}$. On abrège $S_{k}:(z, \tilde{W}) \rightarrow$ $\left(z, S_{k}(z, \tilde{W})\right)$ tel que $S_{k}(z, f(z, \tilde{w}))=f_{k}(z, \tilde{w})$ pour $(z, \tilde{w}) \in \tilde{D}$.

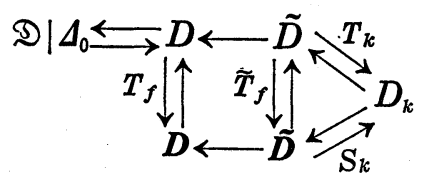

D'après $\left(4.9^{\prime}\right)$ et ( $g 3$ ),on peut appliquer le lemme 2 en plaçant $S_{k}(z, \tilde{W})$ (resp. $\tilde{V}$, resp. $\left.V_{k}\right)$ sur $f\left(z, w_{1}\right)$ (resp. $D_{1}$, resp. $D_{2}$ ). D'après (1) et (2) de ce lemme, la propriété (g2) entraîne que toute composante des surfaces analytiques $\widetilde{A}_{k}$ de $\boldsymbol{D}$ constante pour $z \in \Delta$, d'où que chaque $A_{k}(k=1, \cdots, \mu)$ de $\boldsymbol{D}$ se trouve audessus de l'hyperplan de la forme $\Delta_{0} \times\left\{c_{k}\right\}$ dans $\Delta_{0} \times \boldsymbol{P}^{1}(W)$. On dénote par $G_{k}(z, W)$ la fonction de Green de $D(z)$ avec pôle à $A_{k}(z)=c_{k}$. On a $G_{k}(z, f(z, w))$ $=g_{k}(z, w)$ pour $(z, w) \in D$, et le développement dans un voisinage de $A_{k}$ dans $D$

$$
G_{k}(z, W)=\frac{1}{l_{k}} \log \frac{1}{\left|W-c_{k}\right|}+\Lambda_{k}(z)+\operatorname{Re}\left\{\sum_{n=1}^{\infty} a_{n}(z)\left(W-c_{k}\right)^{n / l_{k}}\right\} .
$$

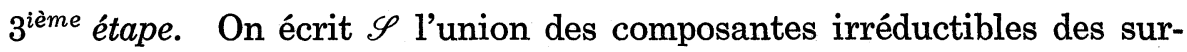
faces de ramification de $D$ au-dessus de $\Delta_{0} \times \boldsymbol{P}^{1}(W)$. On partage ici $\mathscr{S}$ en deux parties disjointes: $\left\{\mathscr{S}_{c}, \mathscr{S}_{v}\right\}$ de façon que chaque composante appartenant à $\mathscr{S}_{c}$ soit constante pour $z \in \Delta_{0}$ et celle appartenant à $\mathscr{S}_{v}$ soit non-constante pour $z \in \Delta_{0}$. Si $\mathscr{S}_{v}=\phi$, il en résulte par (4.10) que le triple $\left(\mathscr{D}\left|\Delta_{0}, \Delta_{0}, \pi\right| \Delta_{0}\right)$ est équivalent à celui trivial $\left(\boldsymbol{D}\left(z_{0}\right) \times \Delta_{0}, \Delta_{0}, \tilde{p}\right)$. C'est contradiction avec la première supposition, d'où $\mathscr{S}_{v}=\left\{s_{s}\right\}_{j=1}^{\nu_{1}}$ tel que $\nu \geqq 1$. On fixe ses regards sur $s_{1}$ de $\mathscr{S}_{v} . \quad m_{1}-1(\geqq 1)$ désigne l'ordre de ramification de $\boldsymbol{D}$ au-dessus de $\Delta_{0} \times \boldsymbol{P}^{1}(W)$ le long de $s_{1}$. On va alors former un autre ouvert connexe $D^{(1)}$ étalé au-dessus de $\Delta_{0} \times \boldsymbol{P}^{1}(W)$ satisfaisant aux conditions: (a) $\boldsymbol{D}$ soit un revêtement de $\boldsymbol{D}^{(1)}$ sans 
point frontière relatif, donc on pose $m^{(1)}$ la multiplicité de $D$ comme revêtement de $D^{(1)} ;(b) p^{(1)}$ désignant la projection de $D$ sur $D^{(1)}$, chaque point de $p^{(1)} \circ s_{1}$ soit celui ordinaire de $\boldsymbol{D}^{(1)}$ comme revêtement de $\Delta_{0} \times \boldsymbol{P}^{1}(W):(c)$ chaque $A_{k}\left(=c_{k}\right)(k=1, \cdots, \mu)$ soit composante des surfaces de ramification de $\boldsymbol{D}$ comme revêtement de $D^{(1)}$ dont l'ordre de ramification est juste $m^{(1)}-1$, donc chaque $l_{k}$ se divise par $m^{(1)}$, soit $l_{k}=m^{(1)} \times n_{k}^{(1)} ;(d) p^{(1)}(z, \cdot)$ désignant la restriction de $p^{(1)}$ sur chaque $D(z)$ où $z \in \Delta_{0}$, et $G_{k}^{(1)}(z, W)$ la fonction de Green de $D^{(1)}(z)$ avec pôle à $p^{(1)} \circ A_{k}(z)$, il vienne $G_{k}^{(1)}\left(z, p^{(1)}(z, W)\right)=m^{(1)} G_{k}(z, W)$ pour $W$ $\in D(z)$.

En effet, on prend dans $D$ les $m_{1}$ points $\left\{P^{(1)}, \cdots, P^{\left(m_{1}\right)}\right\}\left(=\mathscr{E}_{1}\right)$ voisins de $s_{1}$ qui se trouve au-dessus d'un point $\left(z, W_{0}\right) \in \Delta_{0} \times \boldsymbol{P}^{1}(W)$ tel que tous les points de $\boldsymbol{D} \operatorname{sur}\left(z, W_{0}\right)$ soient points ordinaires de $\boldsymbol{D}$ comme revêtement de $\Delta_{0} \times \boldsymbol{P}^{1}(W)$. On trace sur $\boldsymbol{P}^{1}(W)$ une courbe $l: t \rightarrow l(t)$ où $0 \leqq t \leqq 1$ partant de ce point $W_{0}$, ne pas nécessairement de Jordan et ne pas rencontrant la projection sur $\boldsymbol{P}^{1}(W)$ de $\mathscr{S}\left(z_{0}\right)=\left\{s_{j}\left(z_{0}\right)\right\}_{j=1}^{\nu}$. On fait partir chaque point $P^{(i)} \in \mathscr{E}_{1}\left(i=1, \cdots, m_{1}\right)$ le long de $l$ dans $D$ et note désormais $\mathscr{P}_{l}^{(i)}(t)$ le point correspondant à $l(t)$ tel que $\mathscr{P}_{i}^{(i)}(0)=P^{(i)}$. On fait la remarque $1^{\circ}$ : supposons qu'il existe un point de $\mathscr{E}_{1}$, soit $P^{(1)}$, tel que $\mathscr{P}_{l}^{(1)}(t) \in \boldsymbol{D}$ pour $0 \leqq t<1$ mais $\mathscr{P}_{l}^{(1)}(1) \in \partial \boldsymbol{D}\left(=\Delta_{0} \times \partial \boldsymbol{D}\left(z_{0}\right)\right)$. Alors tous les autres $\mathscr{P}_{i}^{(i)}(t)\left(i=2, \cdots, m_{1}\right)$ l'est aussi $\mathscr{P}_{l}^{(i)}(t) \in D$ pour $0 \leqq t<1$ et $\mathscr{P}_{i}^{(i)}(1) \in \partial D$. Car, on regarde l'ensemble $\mathscr{E}_{1}$ de $\boldsymbol{D}$ comme celui de $\tilde{D}$. Prenons arbitrairement un $k$ tel que $1 \leqq k \leqq \mu$. D'après $\left(4.9^{\prime}\right)$ et $(g 3)$, on peut appliquer encore le lemme 2 en plaçant $S_{k}(z, \tilde{W})\left(\right.$ resp. $\tilde{V}$; resp. $\left.V_{k}\right)$ sur $f\left(z, w_{1}\right)$ (resp. $D_{1}$; resp. $D_{2}$ ). D'après l'hypothèse que $s_{1}$ soit non-constante pour $z \in \Delta_{0}$, (4) du lemme 2 entraîne que les $m_{1}$ points de $\mathscr{E}_{1}$ correspondent par la transformation $S_{k}$ aux $m_{1}$ points $\mathscr{E}_{1, k}=\left\{Q_{k}^{(1)}, \cdots, Q_{k}^{\left(m_{1}\right)}\right\}$ voisins de $S_{k} \circ S_{1}=s_{1 k}$ qui se trouvent au-dessus d'un point $\left(z, W_{0 k}\right)$ où $W_{0 k}$ ne dépend pas de $z \in \Delta_{0}$ de facon que $Q_{k}^{(i)}$ $=S_{k} \circ P^{(i)}\left(i=1, \cdots, m_{1}\right)$. Posons $\mathscr{Q}_{k}^{(i)}(t)=S_{k} \circ \mathscr{P}_{l}^{(i)}(t)$ pour $0 \leqq t \leqq 1$ tel que $\mathscr{Q}_{k}^{(i)}(0)$ $=Q_{k}^{(i)}$. Puisque $\tilde{T}_{f}$ et $T_{k}$ sont bijectives, il s'ensuit par l'hypothèse pour $P^{(1)}$ que $\mathscr{Q}_{k}^{(1)}(t) \in D_{k}$ pour $0 \leqq t<1$ mais $\mathscr{Q}_{k}^{(1)}(1) \in \partial D_{k}$. On désigne, en général, pour le point quelconque $Q$ (resp. $P$ ) de $V_{k}$ (resp. $V$ ) par $w_{k} \circ Q$ (resp. $W \circ P$ ) la projection sur $\boldsymbol{P}^{1}\left(w_{k}\right)$ (resp. $\left.\boldsymbol{P}^{1}(W)\right)$ de celle sur $\Delta_{0} \times \boldsymbol{P}^{1}\left(w_{k}\right)\left(\right.$ resp. $\left.\Delta_{0} \times \boldsymbol{P}^{1}(W)\right) \mathrm{du}$ point $Q$ (resp. $P$ ). D'après ( $g 3$ ), on a alors $\left|w_{k} \circ \mathscr{Q}_{k}^{(1)}(t)\right|<1$ pour $0 \leqq t<1$ et $\left|w_{k} \circ \mathscr{Q}_{k}^{(1)}(1)\right|=1$. Il en résulte d'après (4) du lemme 2 que $\left|w_{k} \circ \mathscr{Q}_{k}^{(i)}(t)\right|<1$ pour $0 \leqq t<1$ et $\left|w_{k} \circ \mathscr{Q}_{k}^{(i)}(1)\right|=1\left(i=2, \cdots, m_{1}\right)$. D'après $(g 3)$, on obtient $\mathscr{Q}_{k}^{(i)}(t) \in D_{k}$ pour $0 \leqq t<1$ et $\mathscr{Q}_{k}^{(i)}(1) \in \partial D_{k}$, d'où $\mathscr{P}_{i}^{(i)}(t) \in D$ pour $0 \leqq t<1$ et $\mathscr{P}_{l}^{(i)}(1) \in \partial D$. La remarque $1^{\circ}$ est prouvée. Soit $L: t \rightarrow L(t)$ où $0 \leqq t \leqq 1$ une courbe fermée quelconque sur $\boldsymbol{P}^{1}(W)$ partant du point $W_{0}$ ci-dessus telle que,en faisant partir $\boldsymbol{P}^{(1)}$ de $\mathscr{E}_{1}$ le long de $L$ dans $D$, on ait $\mathscr{P}_{L}^{(1)}(t) \in D$ pour $0 \leqq t \leqq 1$. D'après la remarque $1^{\circ}$, il vient, pour tous les autres $P^{(i)}\left(i=2, \cdots, m_{1}\right)$ de $\mathscr{E}_{1}, \mathscr{P}_{L}^{(i)}(t) \in D$ pour $0 \leqq t \leqq 1$. On écrit $L\left(\mathscr{E}_{1}\right)=\left\{\mathscr{P}_{L}^{(1)}(1), \cdots, \mathscr{P}_{L}^{\left(m_{1}\right)}(1)\right\}$. On va montrer la remarque $2^{\circ}$ : supposons que $\mathscr{E}_{1} \neq L\left(\mathscr{E}_{1}\right)$ et $\mathscr{E}_{1} \cap L\left(\mathscr{E}_{1}\right) \neq \phi$. On abrège $\mathscr{E}_{2}=\mathscr{E}_{1} \cup L\left(\mathscr{E}_{1}\right)=$ 
$\left\{P^{(1)}, \cdots, P^{\left(m_{1}\right)}, P^{\left(m_{1}+1\right)}, \cdots, P^{\left(\rho_{1}\right)}\right\}$ tel que $m_{1}<\rho_{1}<2 m_{1}$. Alors $\mathscr{E}_{2}$ a pour tout $k(=1, \cdots, \mu)$ fixé les propriétés: $(\alpha)$ toutes les valeurs de $G_{k}(z, W)$ aux points de $\mathscr{E}_{2}$ sont egaux; $(\beta)$ s'il existe un point de $\mathscr{E}_{2}$, soit $P^{(1)}$, et une courbe $\gamma: t \rightarrow$ $\gamma(t)$ où $0 \leqq t \leqq 1$ sur $P^{1}(W)$ partant du point $W_{0}$ et arrivant au point $c_{k}\left(=A_{k}\right)$ tel que, en faisant partir $P^{(1)}$ le long de $\gamma$ dans $D$, on ait $\mathscr{P}_{\gamma}^{(1)}(t) \in D$ pour $0 \leqq t$ $\leqq 1$ et $\mathscr{P}_{r}^{(1)}(1)=A_{k}$, alors pour tous les autres $P^{(j)}\left(2 \leqq j \leqq \rho_{1}\right)$, il vient aussi $\mathscr{P}_{\gamma}^{(j)}(t) \in D$ pour $0 \leqq t \leqq 1$ et $\mathscr{P}_{r}^{(j)}(1)=A_{k}$. Car, par définition tous les points de $\mathscr{E}_{2}$ se trouve au-dessus du point $\left(z, W_{0}\right)$. On applique le lemme 2 sous la même configuration que la remarque $1^{\circ}$. Par construction de $\mathscr{E}_{2}$ et (1) du lemme 2, en posant $Q_{k}^{(j)}=S_{k}\left(P^{(j)}\right)\left(j=1, \cdots, \rho_{1}\right), Q_{k}^{(j)}$ se trouve au-dessus du point $\left(z, W_{0 k}\right)$ tel que $W_{0 k}$ soit indépendant de $z \in \Delta_{0}$. Par définition de $S_{k}$, on a $G_{k}(z, W)=$ $-\log \left|S_{k}(z, \tilde{W})\right| \operatorname{pour}(z, W) \in V$. Il en résulte que toutes les valeurs de $G_{k}$ aux points $P^{(j)}\left(j=1, \cdots, \rho_{1}\right)$ sont égaux à $-\log \left|W_{0 k}\right|$, d'où $(\alpha)$. On écrit $\mathscr{Q}_{\gamma k}^{(j)}(t)$ $=S_{k}\left(\mathscr{P}_{r}^{(j)}(t)\right)$ pour $0 \leqq t \leqq 1$. D'après l'hypothèse de $(\beta)$ pour $P^{(1)}$ et les propriétés (g2), ( $g 3)$, il vient $\left|w_{k} \circ \mathscr{Q}_{\gamma k}^{(1)}(t)\right| \leqq 1$ pour $0 \leqq t \leqq 1$ et $\left|w_{k} \circ \mathscr{Q}_{\gamma k}^{(1)}(1)\right|=0$. Par (4) du lemme 2 et $\mathscr{E}_{1} \cap L\left(\mathscr{E}_{1}\right) \neq \phi$, on a aussi $\left|w_{k} \circ \mathscr{Q}_{r k}^{(j)}(t)\right| \leqq 1$ pour $0 \leqq t \leqq 1$ et $\left|w_{k} \circ \mathscr{Q}_{\gamma k}^{(j)}(1)\right|=0\left(j=2, \cdots, \rho_{1}\right)$. Par $(g 2),(g 3)$, il vient $\mathscr{Q}_{\gamma k}^{(j)}(t) \in D_{k}$ pour $0 \leqq t \leqq 1$ et $T_{k}^{-1} \circ \mathscr{Q}_{\gamma k}^{(j)}(1) \in \tilde{\alpha}_{k}$. Il s'ensuit que $\mathscr{P}_{r}^{(j)}(t) \in D$ pour $0 \leqq t \leqq 1$ et $\mathscr{P}_{\gamma}^{(j)}(1)=A_{k}$, d'où $(\beta)$.

On considère de nouveau $\mathscr{E}_{2}$ comme $\mathscr{E}_{1}$ précédent. S'il existe une courbe $L_{1}$ vérifiant à la même condition dans la remarque $2^{\circ}$ que $L$, on obtient aussi $\mathscr{E}_{3}=\mathscr{E}_{2} \cup L\left(\mathscr{E}_{2}\right)=\left\{\boldsymbol{P}^{(1)}, \cdots, P^{\left(\rho_{2}\right)}\right\}$ tel que $\rho_{1}<\rho_{2}<2 \rho_{1}$. Alors $\mathscr{E}_{3}$ a pareillement les propriétés $(\alpha),(\beta)$ pour $\rho_{2}$ au lieu de $\rho_{1}$. On répète cette procédure et arrive à un nombre fini, soit $m^{(1)}$, de points $\left\{P^{(1)}, \cdots, P^{(m(1)}\right\}\left(=\mathscr{E}^{(1)}\right)$ de $\boldsymbol{D}$ avec $W \circ P^{(j)}=W_{0}\left(j=1, \cdots, m^{(1)}\right)$ indépendant de $z \in \Delta_{0}$, ayant les propriétés $(\alpha)$, $(\beta)$ pour $m^{(1)}$ au lieu de $\rho_{1}$, et de plus celle suivante $(\gamma)$ : pour chaque courbe fermée $l: t \rightarrow l(t)$ sur $P^{1}(W)$ partant de $W_{0}$, il arrive trois cas: 1) il existe $t_{0}$ où $0 \leqq t_{0}<1$ tel que pour tout $j=1, \cdots, m^{(1)}$ on ait $\mathscr{P}_{l}^{(j)}(t) \in D$ pour $0 \leqq t<t_{0}$ mais $\mathscr{P}_{l}^{(j)}\left(t_{0}\right) \in \partial D$; 2) $l\left(\mathscr{E}^{(1)}\right)=\mathscr{E}^{(1)}$; 3) $l\left(\mathscr{E}^{(1)}\right) \cap \mathscr{E}^{(1)}=\phi$. Maintenant, puisque tous les points $P^{(j)}$ de $\mathscr{E}^{(1)}$ est point ordinaire de $\boldsymbol{D}$ comme revêtement de $\Delta_{0} \times \boldsymbol{P}^{1}(W)$, il existe (si nécessaire, il suffit de reprendre plus petit $\Delta_{0}$ ) un polydisque dans $\boldsymbol{D}$ de la forme $\Delta_{0} \times \delta$ où $\delta=\left\{\left|W-W_{0}\right|<\tau\right\}\left(\subset \boldsymbol{P}^{1}(W)\right)$ tel que chaque feuille $V^{(j)}$ $\left(j=1, \cdots, m^{(1)}\right)$ de $D$ étalé au-dessus de $\Delta_{0} \times \delta$ contenant $P^{(j)}$ soit univalente. On en identifie les $m^{(1)}$ points $\left(z, W^{(j)}\right) \in V^{(j)}$ avec même projection $(z, W)$ sur $\Delta_{0} \times \delta$, et lui donne les coordonnées $(z, W) \in \Delta_{0} \times \delta$. On prolonge analytiquement cette identification dans $\boldsymbol{D}$ le long de courbe quelconque sur $\boldsymbol{P}^{1}(W)$ partant du point $\left(z, W_{0}\right)$. Par la propriété $(\gamma)$, elle détermine l'ouvert connexe $\boldsymbol{D}^{(1)}$ étalé au-dessus de $\Delta_{0} \times \boldsymbol{P}^{1}(W)$. C'est $\boldsymbol{D}^{(1)}$ qu'on désire au début de la $3^{\text {ième }}$ étape. Car, d'apres $(\gamma), D^{(1)}$ satisfait à la condition $(a)$. D'après $\mathscr{E}^{(1)} \supset \mathscr{E}_{1}$, il s'ensuit par constraction de $D^{(1)}$ que $D$ satisfait à $(b)$. La propriété $(\beta)$ pour $\mathscr{E}^{(1)}$ entraîne évidemment $(c)$ pour $D^{(1)}$. Pour montre $(d)$, on pose $H_{k}^{(1)}=$ 
$G_{k} \circ\left(p^{(1)}\right)^{-1} \operatorname{sur} D^{(1)}$. Il vient d'après $(\alpha)$ que $H_{k}^{(1)}$ définit une fonction uniforme dans $D^{(1)}$ telle que $H_{k}^{(1)}$ soit nulle sur $\partial D^{(1)}$ et la restriction de $H_{k}^{(1)}$ sur chaque $D^{(1)}(z)$ soit harmonique sauf au point $p^{(1)} \circ A_{k}(z)$. Puisque $p^{(1)} \circ A_{k}(z)$ se trouve au-dessus de $\left(z, c_{k}\right)$, il s'ensuit par notation $(c)$ que $t=\left(W-c_{k}\right)^{1 / n_{k}^{(1)}}$ est le paramètre local d'un point $\left(p^{(1)} \circ A_{k}\right)(z)$ de $D^{(1)}(z)$, d'où, d'après (4.11), $m^{(1)} \times$ $H_{k}^{(1)}(z, W)$ n'est autre que la fonction de Green $G_{k}^{(1)}(z, W)$ de $D^{(1)}(z)$ avec pôle à $p^{(1)} \circ A_{k}(z)$, d'où $(d)$.

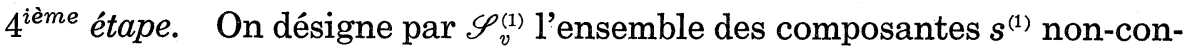
stante pour $z \in \Delta_{0}$ des surfaces de ramification de $D^{(1)}$ comme revêtement de $\Delta_{0} \times \boldsymbol{P}^{1}(W)$. On a, d'après $(a),(b)$ de $\boldsymbol{D}^{(1)}, \mathscr{S}_{v} \supseteq \mathscr{S}_{v}^{(1)}$. Si $\mathscr{S}_{v}^{(1)} \neq \phi$, on répète la même procédé à $D^{(1)}$ et $s^{(1)} \in \mathscr{S}_{v}^{(1)}$ que celui faite à $D$ et $s_{1} \in \mathscr{S}_{v}$, et obtient un ouvert connexe $\boldsymbol{D}^{(2)}$ étalé au-dessus de $\Delta_{0} \times \boldsymbol{P}^{1}(W)$ satisfaisant aux conditions correspondantes $(a),(b),(c),(d)$ en plaçant $\boldsymbol{D}^{(1)}\left(\right.$ resp. $\left.\boldsymbol{D}^{(2)}\right) \operatorname{sur} \boldsymbol{D}\left(\right.$ resp. $\left.\boldsymbol{D}^{(1)}\right)$. En nombre fini, au plus $\nu$, fois de telles répétitions, on arrive à $D^{(s)}$ satisfaisant à celles correspondantes $(a),(b),(c),(d)$ tel que $\mathscr{S}_{v}^{(s)}=\phi . \quad$ Donc $D$ est égale à l'espace produit $\Delta_{0} \times D^{(s)}\left(z_{0}\right)$. En partant de $D^{(s)}$ et remontant $D^{(s-1)}, \cdots, D^{(1)}$ et enfin $D$, on prend $D^{(s)}\left(z_{0}\right)$ (resp. $\Delta_{0}$; resp. $m^{(s)} \times \cdots \times m^{(1)}$; resp. $A_{k}\left(=c_{k}\right)$ ) comme $R$ (resp. $\Delta$; resp. $m$; resp. $\beta_{k}$ ) du lemme 3 . Car, d'après $(a)$ pour chaque $D^{(k)}(0 \leqq k \leqq s)$ la propriété $(1)$ de ce lemme est vérifiée. D’après $(c)$ pour chaque $D^{(k)},(2)$ est aussi vérifiée. D'après $(d)$, pour chaque $\alpha_{k}(k=1, \cdots, \mu)$, il vient pour $(z, W) \in D, G_{k}(z, W)=\left(m^{(1)} \times \cdots \times m^{(s)}\right)^{-1} \cdot G_{k}^{(s)}\left(z, p^{(s)} \circ \cdots \circ p^{(1)}(z, W)\right)$. Le second membre est, d'après $D^{(s)}=\Delta_{0} \times D^{(s)}\left(z_{0}\right)$, indépendant de $z \in \Delta^{0}$, d'où l'expression (4.11) devient pour $(z, W)$ voisin de $A_{k}$ dans $\boldsymbol{D}$

$$
G_{k}(z, W)=\frac{1}{l_{k}} \log \frac{1}{\left|W-c_{k}\right|}+\Lambda\left(z_{0}\right)+\operatorname{Re}\left\{\sum_{n=1}^{\infty} a_{n}\left(z_{0}\right)\left(W-c_{k}\right)^{n / l_{k}}\right\} .
$$

La propriété (3) étant visible, on a prouvé le lemme 3 pour $\Delta=\Delta_{0}$.

Soit $z \in \Delta$ et $g_{i}(z, w)(i=1,2)$ la fonction de Green de $\mathfrak{D}(z)$ avec pôle au point $\alpha_{i}(z)$. Alors $\omega_{i}(z, \omega)=\left(\partial g_{i}(z, \omega) / \partial \omega\right) d \omega$ est 1-forme méromorphe pour $w \in$ ( $(z)$, et le ratio $F(z, w)=\omega_{2}(z, w) / \omega_{1}(z, w)$ bien définit une fonction uniforme méromorphe pour $w \in \mathfrak{D}(z)$. On considère la transformation de $\mathfrak{D}, T_{F}:(z, w)$ $\rightarrow(z, F(z, w))$ et note $D^{*}$ l'image de $\mathfrak{D}$ par $T_{F^{*}} . \quad D^{*}$ est un ouvert connexe étale au-dessus de $\Delta \times \boldsymbol{P}^{1}$. On a ainsi un triple $\left(D^{*}, \Delta, p^{*}\right)$, $p^{*}$ étant la première projection. En autre côté, par (4.9), la restriction sur $\Delta_{0}$ de ce triple: $\left(D^{*} \mid \Delta_{0}\right.$, $\left.\Delta_{0}, p^{*} \mid \Delta_{0}\right)$ n'est autre que $(D, \Delta, p)$ qui vient d'être discuté. Il en suffit pour le lemme 3 de prendre $D=D^{*}, R=D^{(s)}\left(z_{0}\right), m=m^{(1)} \times \cdots \times m^{(s)}, p=p^{*}$ et $\beta_{k}=$ $A_{k}\left(=c_{k}\right)$.

C.Q.F.D.

Si $\mu=1$ au lieu de la condition (i) $\mu \geqq 2$ dans le lemme 3 , la conclusion n'est pas vraie. En utilisant le lemme 3, on montre la réciproque cité en $\mathrm{p}$. 18 comme suit. 
ThÉORÈme 2. Soit $(\mathfrak{D}, \Delta, \pi)$ un triple satisfaisant aux conditions $(L),(T)$ de type topologique $(p, q)$ et soit $d s_{\circledast}^{2}$ la pseudo-métrique de $\supseteqq$ induite par la constante de Robin. Supposons qu'il existe $\mu$ sections analytiques $\alpha_{k}(k=1, \cdots, \mu)$ de $\supseteqq$ sur $\Delta$ telles que la restriction $d s_{Ð}^{2} \mid \alpha_{k}(\Delta)$ de $d s_{\circledast}^{2}$ sur la surface analytique $\alpha_{k}(\Delta)$ de $\mathfrak{D}$ soit identiquement nulle. Alors, si $\mu \geqq 2 p+q-1$, le triple $(\mathfrak{D}, 4, \pi)$ est équivalent à celui trivial sauf les cas suivants: $(1)(p, q)=(0,1) ;(2)(p, q)=$ $(0,2) ;(3) p \geqq 1$ est la double $\mathfrak{D}(z)$ de chaque fibre $\mathfrak{D}(z)$ où $z \in \Delta$ soit hyperelliptique comme surface de Riemann compacte. Ces cas exceptionnels, il faut $\mu \geqq 2 p+q$ pour la pareille.

PREuve. D'après l'hypothèse que $d s^{2} \mid \alpha_{k}(\Delta)$ soit identiquement nulle, la constante de Robin $\lambda_{k}(z)$ de chaque $\mathfrak{D}(z)$ où $z \in \Delta$ par rapport au point $\alpha_{k}(z)$ et aux $\pi$-coordonnées $(z, w)$ de $\supseteqq$ est pluri-harmonique pour $z \in \Delta$. Dans le cas exceptionnelle (1), toute fibre $\mathscr{D}(z)$ est simplement connexe et $\mu \geqq 1$. Alors la

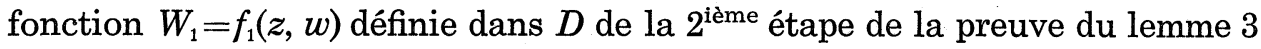
est holomorphe et uniforme $\boldsymbol{D}$ se transforme bijectivement et analytiquement par $T_{1}:(z, w) \rightarrow\left(z, f_{1}(z, w)\right)$ sur l'espace produit $\Delta_{0} \times U$ où $U=\left\{\left|w_{1}\right|<1\right\}$ de façon que $T_{1} \circ A_{1}=\Delta_{0} \times\{0\}$. Il s'ensuit que le triple $(\mathfrak{D}, \Delta, \pi)$ est équivalent à celui trivial $\left(\Delta \times U_{0}, \Delta, p\right), p$ étant la première projection. On exclut désormais ce cas (1). Il en vient $\mu \geqq 2$. On va prouver le théorème par l'absurdité. Supposons maintenant que $(\mathfrak{D}, \Delta, \pi)$ ne soit pas équivalent à celui trivial. D'après le lemme $3,(\mathfrak{D}, \Delta, \pi)$ est équivalent à $(\boldsymbol{D}, \Delta, \boldsymbol{p})$ énoncé dans ce lemme. Soit $p_{0}$ le genre de la surface de Riemann $R$ du lemme 3 et $q_{0}$ le nombre des composantes frontières de $R$. On abrège $\chi_{0}=2 p_{0}+q_{0}-1$ (resp. $\left.\chi=2 p+q-1\right)(\geqq 0)$, le genre du double compact $\bar{R}$ (resp. $\overline{D(0)})$ de $R$ (resp. $D(0)$ ). $\quad D$ est, d'après (2) du lemme 3 , un revêtement de $\Delta \times R$ tel qu'il existe $\mu$ surfaces $\beta_{k}(k=1, \cdots, \mu)$ de $\boldsymbol{D}$ dont l'ordre de ramification est $m-1(\geqq 1)$. On note $m^{*}$ la somme des nombres des autres surfaces de ramification rendu compte d'ordre de $\boldsymbol{D}$ audessus de $\Delta \times R$. D'après (3) du lemme $3, m^{*} \geqq 1$. Il s'ensuit par la relation de Riemann-Hurwitz que

$$
2 \chi-2=m\left(2 \chi_{0}-2\right)+2 \mu \cdot(m-1)+2 m^{*} .
$$

Elle s'arrange

$$
0=m \chi_{0}+(m-2) \chi+(m-1)[\mu-(\chi+1)]+m^{*} .
$$

En les cas exceptionnels (2), (3), on a par $\mu \geqq \chi+1$ que $0 \geqq m \chi_{0}+(m-2) \chi+m^{*}$. Puisque $\chi_{0} \geqq 0, \chi \geqq 1$ et $m \geqq 2$, on a $m^{*}=0$, qui est contradiction. Il s'agit le cas reste tel que $\mu \geqq \chi \geqq 2$. L'expression (4.12) entraîne que $0 \geqq m \chi_{0}+m-3+$ $m^{*}$. D'après $\chi_{0} \geqq 0, m \geqq 2$ et $m^{*} \geqq 1$, il n'y a un seul cas possible: $\left(\chi_{0}, m\right)=(0,2)$. Alors la surface $R$ se regarde, d'après $\chi_{0}=0$, comme un disque unité $U=$ $\{|w|<1\}$ du plan $w$. Il s'ensuit d'après (1), (2) du lemme 3 , et $m=2$ que le 
double $\overline{D(z)}$ de chaque $D(z)$ où $z \in \Delta$ est hyperelliptique. Ce cas est contenu dans (3), qui est contradition. On a demontré le théorème 2.

Le cas (3) tel que $\mu=2 p+q-1$, il existe un triple (I), $\Delta, \pi)$ ne pas équivalent à celui trivial. Il suffit pour cela de prendre comme $\mathfrak{D}$ un ouvert connexe doublement étalé au-dessus de $\Delta \times U$ où $U=\{|w|<1\}$ sans point frontière relatif tel que, entre $2 p+q$ (où $q=1$ ou 2 ) surfaces de ramification de $\mathfrak{D}$, ceux de $2 p+q-1$ soient constantes pour $z \in \Delta$ et l'autre seule non-constante pour $z \in \Delta$.

\section{§5. Variations de solutions du problème de Dirichlet}

Soit $(\mathfrak{D}, \Delta, \pi)$ un triple satisfaisant aux conditions $(L),(T)$. Soit donnée une fonction $f(z, \zeta)$ à valeur complexe définie sur $\partial \Im$ vérifiant à la condition

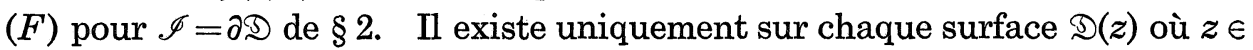
$\Delta$, la solution $u=H_{f}$ du problème de Dirichlet par rapport à la fonction frontière $f$, c'est-à-dire, $u$ vérifie à la condition $(H)$ pour $\mathscr{V}(z)=\mathscr{D}(z)$ de $\S 2$. On a alors la

Formule 3. Pour la section analytique $\alpha$ de $\mathfrak{D}$, il vient

$$
\frac{\partial^{2}}{\partial z_{i} \partial \bar{z}_{j}} H_{f}(z, \alpha(z))=-\frac{2}{\pi} \iint_{D(z)}\left(\frac{\partial^{2} H_{f}}{\partial z_{i} \partial \bar{w}} \frac{\partial^{2} g_{\alpha}}{\partial \bar{z}_{j} \partial w}+\frac{\partial^{2} H_{f}}{\partial \bar{z}_{j} \partial w} \frac{\partial^{2} g_{\alpha}}{\partial z_{i} \partial \bar{w}}\right) d u d v .
$$

Preuve. D'après le lemme 1 , le second membre, comme le premier, est un nombre complexe fini invariant pour les triples équivalents à $(\mathfrak{D}, \Delta, \pi)$. Soit $z_{0} \in \Delta$. On prend un ouvert connexe $D(\Subset U)$ étale au-dessus de $\Delta_{0} \times C$, $\Delta_{0}$ étant un polydisque de centre $z_{0}$ et $C=\{|w|<\infty\}$ tel que $\left(D, \Delta_{0}, p\right), p$ étant la projection de $D$ sur $\Delta_{0}$, soit équivalent à $\left(\mathfrak{D}\left|\Delta_{0}, \Delta_{0}, \pi\right| \Delta_{0}\right)$ et satisfasse à la condition $(R)$ de $\S 2$ et $(R 4)$ de $\S 3$. Puisque $\partial D$ (resp. $f$ ) est analytique $a u$ sens réel dans $U$ (resp. $\partial D$ ), il résulte d'après $(R 1)$ que toute fonction $u(z, w)$ $=H_{f}(z, w)$ (où $z \in \Delta_{0}$ ) définie sur $D(z)$ se regarde comme celle harmonique sur $D\left(z_{0}\right) . \quad g_{0}\left(z_{0}, w\right)$ désignant la fonction de Green sur $D\left(z_{0}\right)$ avec pôle au point 0 cité à $(R 4)$, il vient pour $z \in \Delta_{0}$

$$
u(z, 0)=-\frac{1}{2 \pi} \int_{\partial D\left(z_{0}\right)} u(z, w) \frac{\partial g_{0}\left(z_{0}, w\right)}{\partial n_{w}} d s_{w} .
$$

D'après le même raisonnement que (3.6) entraîne (3.10), on a

$$
\frac{\partial^{2} u}{\partial z_{i} \partial \bar{z}_{j}}\left(z_{0}, 0\right)=-\frac{2}{\pi} \iint_{D\left(z_{0}\right)}\left\{\frac{\partial^{2} u}{\partial z_{i} \partial \bar{w}} \frac{\partial^{2} g_{0}}{\partial \bar{z}_{j} \partial w}+\frac{\partial^{2} u}{\partial \bar{z}_{j} \partial w} \frac{\partial^{2} g_{0}}{\partial z_{i} \partial \bar{w}}\right\}_{\left(z_{0}, w\right)} d u d v
$$

D'après $(R 4)$, cette égalité n'est autre que la formule 3 pour $z=z_{0}$. C.Q.F.D. 
Maintenant, soient $Q_{0}$ et $Q_{1}$ deux points quelconques d'une composante frontière de $\partial \mathfrak{S}(0)$. Considérons l'arc $\mathscr{I}(0)$ de cette composante partant de $Q_{0}$ et arrivant à $Q_{1}$ avec la même direction que $\partial \Im(0)$. Comme $\S 2$, on pose pour $z \in \Delta, \mathscr{I}(z)=\{\Xi(Q, z) \in \partial \mathscr{S}(z): Q \in \mathscr{I}(0)\}$. Sur chaque $\mathscr{D}(z)$ où $z \in \Delta$, on forme la mesure harmonique $\omega\left(z, w: Q_{0}, Q_{1}\right)(=\omega(z, w))$ par rapport à $\mathscr{I}(z)$, c'est-à-dire, $\omega(z, w)$ est harmonique et bornée pour $w \in \mathfrak{D}(z)$ telle que $\omega(z, w)$ $=1$ (resp. 0) pour $w \in \mathscr{I}(z)-\left\{\boldsymbol{E}\left(Q_{0}, z\right), \boldsymbol{\Xi}\left(Q_{1}, z\right)\right\}$ (resp. $\left.\in \partial \mathfrak{D}(z)-\mathscr{I}(z)\right)$. On a alors

Formule $3^{\prime}$. Pour la section analytique $\alpha$ de $\mathfrak{D}$, il vient

$$
\frac{\partial^{2} \omega\left(z, \alpha(z): Q_{0}, Q_{1}\right)}{\partial z_{i} \partial \bar{z}_{j}}=-\frac{2}{\pi} \iint_{D(z)}\left(\frac{\partial^{2} \omega}{\partial z_{i} \partial \bar{w}} \frac{\partial^{2} g_{\alpha}}{\partial \bar{z}_{j} \partial w}+\frac{\partial^{2} \omega}{\partial \bar{z}_{j} \partial w} \frac{\partial^{2} g_{\alpha}}{\partial z_{i} \partial \bar{w}}\right) d u d v
$$

Preuve. Puisque la 1-forme méromorphe $(\partial \omega / \partial w) d w$ sur chaque $\mathscr{P}(z) \cup$ $\partial \mathfrak{D}(z)$ où $z \in \Delta$ satsfait aux conditions $(e 1),(e 2)$ de $\S 2$, le second membre est certainement un nombre complexe fini invariant pour les triples équivalents à $(\mathfrak{D}, \Delta, \pi)$. On suppose, par additivité linaire des deux côtés de la formule $3^{\prime}$ par rapport aux arcs, que l'arc $\mathscr{I}(0)$ soit très petit tel que, pour tout $z_{0}$ fixé dans $\Delta$, il existe par la remarque 2 des $\pi$-coordonnées $\left(\Delta_{0}^{\prime}, \delta\right)$ où $\Delta_{0}^{\prime}$ est un polydisque de centre $z_{0}$ dans $\Delta$ et $\delta=\{|\boldsymbol{w}|<1\}$, d'un voisinage $\mathscr{V}$ de $\mathscr{I}\left(z_{0}\right)$ dans $\hat{\mathfrak{D}}$ tel que $\mathscr{V} \cap \mathfrak{D}$ (resp. $\mathscr{V} \cap \partial \supseteqq$ ) corresponde à l'ensemble produit $\Delta_{0}^{\prime} \times\{\boldsymbol{w} \in \delta: \operatorname{Im} \boldsymbol{w}$ $>0\}$ (resp. $\Delta_{0}^{\prime} \times\{w \in \delta: \operatorname{Im} w=0\}$ ). Soit en nouveau $z_{0} \in \Delta$ et prenons l'ouvert $D$ au-dessus de $\Delta_{0} \times C$ et la fonction de Green $g_{0}\left(z_{0}, w\right)$ mentionnés dans la preuve de la formule 3. On dénote par $\zeta_{0}$ (resp. $\zeta_{1}$ ) le point de $\partial D\left(z_{0}\right)$ correspondant à $\Xi\left(Q_{0}, z_{0}\right)$ (resp. $\left.\Xi\left(Q_{1}, z_{0}\right)\right)$ de $\partial \mathfrak{D}\left(z_{0}\right)$ et pose $I\left(z_{0}\right)$ l'arc de $\partial D\left(z_{0}\right)$ entre $\zeta_{0}$ et $\zeta_{1}$. On sait alors que, pour $z \in \Delta_{0}, I(z)=\left\{\xi_{0}(z, \zeta): \zeta \in I\left(z_{0}\right)\right\}$ de $\partial D(z)$ corresponde $\mathscr{I}(z)$ de $\partial \mathfrak{D}(z)$. D'après $(2.7)$, il vient pour $\zeta \in \partial D\left(z_{0}\right)-\left\{\zeta_{0}, \zeta_{1}\right\}$,

$$
\left\{\frac{\partial^{2}}{\partial z_{i} \partial \bar{z}_{j}} \omega(z, \zeta)\right\}_{z=z_{0}}=\left\{\frac{\partial^{2} \omega}{\partial z_{i} \partial \bar{w}} \frac{\partial g_{0}}{\partial \bar{z}_{j}} / \frac{\partial g_{0}}{\partial \bar{w}}\right\}_{\left(z_{0}, \zeta\right)}+\left\{\frac{\partial^{2} \omega}{\partial \bar{z}_{j} \partial w} \frac{\partial g_{0}}{\partial z_{i}} / \frac{\partial g_{0}}{\partial w}\right\}_{\left(z_{0}, \zeta\right)} .
$$

On considère les $\pi$-coordonnées $\left(\Delta_{0}^{\prime}, \delta\right)$ ci-dessus tel que $\Delta_{0}^{\prime} \subset \Delta_{0}$. Il y a une relation analytique $(z, w) \rightarrow(z, w(z, w))$ entre un voisinage $V$ de $I\left(z_{0}\right)$ dans $U$ et $\Delta_{0}^{\prime} \times \delta$. Par la remarque 2, l'ensemble analytique $\left\{\xi_{0}\left(z, \zeta_{0}\right): z \in \Delta_{0}^{\prime}\right\}$ (resp. $\left.\left\{\xi_{0}\left(z, \zeta_{1}\right): z \in \Delta_{0}^{\prime}\right\}\right)$ de $V$ corresponde au produit $\Delta_{0}^{\prime} \times\left\{c_{0}\right\}$ (resp. $\left.\Delta_{0}^{\prime} \times\left\{c_{1}\right\}\right)$ de $\Delta_{0}^{\prime} \times \delta$ tel que $c_{0}$ (resp. $c_{1}$ ) soit une constante réelle indépendante de $z \in \Delta_{0}^{\prime}$, Il s'ensuit que pour $(z, w) \in V$

$$
\omega(z, w)=\frac{1}{\pi} \arg \left(\frac{w-c_{1}}{w-c_{0}}\right)+h(z, w) \quad \text { où } w=w(z, w),
$$

$h(z, w)$ étant fonction analytique au sens réel dans $\Delta_{0}^{\prime} \times \delta$ et harmonique par rapport à $w$ pour $z$ fixé dans $\Delta_{0}^{\prime}$, d'où que pour $(z, w) \in V$, 


$$
\frac{\partial^{2} \omega(z, w)}{\partial z_{i} \partial \bar{z}_{j}}=\frac{\partial^{2} h(z, \boldsymbol{w}(z, w))}{\partial z_{i} \partial \bar{z}_{j}} ; \frac{\partial^{2} \omega(z, w)}{\partial z_{i} \partial \bar{w}}=\frac{\partial^{2} h(z, w(z, w))}{\partial z_{i} \partial \bar{w}}
$$

On sait que, bien que $\omega(z, w)$ soit fonction dans un voisinage de $D \cup \partial D$ dans $U$ avec singularité le long des ensembles analytiques $\xi_{0}\left(\Delta_{0}, \zeta_{0}\right)$ et $\xi_{0}\left(\Delta_{0}, \zeta_{1}\right)$, ses différentielles de la forme $\partial^{2} \omega / \partial z_{i} \partial \bar{z}_{j}$ ou $\partial^{2} \omega / \partial z_{i} \partial \bar{w}$ sont fonctions analytiques au sens réel sans singularité dans ce voisinage de $D \cup \partial D$ dans $U$. Il en résulte que toutes les fonctions de deux côtés de (5.1) sont continues même au point $\zeta=\zeta_{0}$ ou $\zeta_{1}$ sur $\partial D\left(z_{0}\right)$. Par intégration de (5.1) multipliée par $(-1 / 2 \pi)\left(\partial g_{0}\left(z_{0}, \zeta\right) / \partial n_{\zeta}\right) d s_{\zeta}$ le long de $\partial D\left(z_{0}\right)$, on a

$$
\begin{aligned}
&-\frac{1}{2 \pi} \int_{\partial D\left(z_{0}\right)}\left\{\frac{\partial^{2}}{\partial z_{i} \partial \bar{z}_{j}} \omega(z, \zeta)\right\}_{z=z_{0}} \frac{\partial g_{0}\left(z_{0}, \zeta\right)}{\partial n_{\zeta}} d s_{\zeta} \\
&=-\frac{1}{2 \pi} \int_{\partial D\left(z_{0}\right)}\left\{\left(\frac{\partial^{2} \omega}{\partial z_{i} \partial \bar{w}} \frac{\partial g_{0}}{\partial \bar{z}_{j}} / \frac{\partial g_{0}}{\partial \bar{w}}\right)\right. \\
&\left.+\left(\frac{\partial^{2} \omega}{\partial z_{j} \partial w} \frac{\partial g_{0}}{\partial z_{i}} / \frac{\partial g_{0}}{\partial w}\right)\right\}_{\left(z_{0}, \zeta\right)} \frac{\partial g_{0}\left(z_{0}, \zeta\right)}{\partial n_{\zeta}} d s_{\zeta}
\end{aligned}
$$

Puisque $\left(\partial^{2} \omega / \partial z_{i} \partial \bar{z}_{j}\right)\left(z_{0}, w\right)$ étant harmonique et continue pour $w \in D\left(z_{0}\right) \cup$ $\partial D\left(z_{0}\right)$, le premier membre de (5.2) est égale à $\left(\partial^{2} \omega / \partial z_{i} \partial \bar{z}_{j}\right)\left(z_{0}, 0\right)$, qui n'est autre que le premier membre de la formule $3^{\prime}$ pour $z=z_{0}$. D'après le même raisonnement que la formule 3 , le seconde de (5.2) devient

$$
-\frac{2}{\pi} \iint_{D\left(z_{0}\right)}\left\{\left(\frac{\partial^{2} \omega}{\partial z_{i} \partial \bar{w}} \frac{\partial^{2} g_{0}}{\partial \bar{z}_{j} \partial w}\right)+\left(\frac{\partial^{2} \omega}{\partial \bar{z}_{j} \partial w} \frac{\partial^{2} g_{0}}{\partial z_{i} \partial \bar{w}}\right)\right\}_{\left(z_{0}, w\right)} d u d v
$$

qui est égale au second de la formule $3^{\prime}$ pour $z=z_{0}$. On a obtenu l'égalité de la formule $3^{\prime}$ pour $z=z_{0}$.

\section{§6. Variations d'intégrales de Dirichlet}

Soit $R$ une surface de Riemann à bord $\partial R$ et soit $F$ une fonction à valeur complexe de classe $C^{2}$ dans $R \cup \partial R$. Les suivants sont connus: l'intégrale de $F$ étendue à $R$ définie par

$$
I_{R}(F)=\iint_{R}\left|\frac{\partial F}{\partial w}\right|^{2} d u d v
$$

est dite celle de Dirichlet; l'aire totale $S_{\partial R}(F)$ (ne pas nécessairement positive) des ouverts bornés par les courbes $F(\partial R)$ s'exprime

$$
S_{\partial R}(F)=\frac{\sqrt{-1}}{2} \int_{\partial R} F d \bar{F}=\iint_{R}\left(\left|\frac{\partial F}{\partial w}\right|^{2}-\left|\frac{\partial F}{\partial \bar{w}}\right|^{2}\right) d u d v
$$

il vient 


$$
I_{R}(F)=S_{\partial R}(F)+\iint_{R}\left|\frac{\partial F}{\partial \bar{w}}\right|^{2} d u d v ;
$$

$I_{R}(F) \geqq S_{\partial R}(F)$ pour toute $F$, et l'égalité est vraie si et seulement si $F$ est analytique dans $R$.

Maintenant on retourne au triple (D, $\Delta, \pi)$ vérifiant aux conditions $(L)$, $(T)$. On se soumis à la même configuration que celle de la formule $3: f$ (resp. $\left.H_{f}\right)$ sur $\partial \mathfrak{D}($ resp. $\mathscr{D} \cup \partial \mathfrak{D})$ satisfaisant à la condition $(F)$ (resp. $\left.(H)\right)$. On dénote par $f(z, \cdot)\left(\right.$ resp. $\left.H_{f}(z, \cdot)\right)$ la restriction de $f\left(\right.$ resp. $\left.H_{f}\right)$ sur $\partial \mathscr{D}(z)($ resp. $\mathscr{D}(z) \cup$ $\partial \mathscr{D}(z)$ ) (où $z \in \Delta$ ) et on pose simplement pour $z \in \Delta$

$$
I(f, z)=I_{\Phi(z)}\left(H_{f}(z, \cdot)\right) ; \quad S(f, z)=S_{\partial \Phi(z)}(f(z, \cdot)) .
$$

Alors on a par notation $\partial f / \partial z_{i}$ sur $\partial \subseteq$ de $\S 2$, la

Formule 4. Pour toute fonction $f$ satisfaisant à la condition $(F)$, il vient

$$
\begin{aligned}
& \frac{\partial^{2} I(f, z)}{\partial z_{i} \partial \bar{z}_{j}}=\frac{\sqrt{-1}}{2} \int_{\partial D(z)} \frac{\partial f}{\partial z_{i}} d\left(\overline{\frac{\partial f}{\partial z_{j}}}\right) \\
& +\iint_{\mathscr{D}(z)}\left(\frac{\partial^{2} H_{f}}{\partial z_{i} \partial \bar{w}} \frac{\partial^{2} H_{\mathcal{J}}}{\partial \bar{z}_{j} \partial w}+\frac{\partial^{2} H_{\vec{J}}}{\partial z_{i} \partial \bar{w}} \frac{\partial^{2} H_{f}}{\partial \bar{z}_{j} \partial w}\right) d u d v .
\end{aligned}
$$

DÉmonstration. Tous les termes de cette formule sont invariants pour les triples équivalents à $(\mathfrak{D}, \Delta, \pi)$. Soit $z_{0} \in \Delta$ et soit $D(\subset U)$ mentionné dans la preuve de la formule 3 . On abrège $H_{f}(z, w)=u(z, w)$ pour $(z, w) \in D ; \partial D(z)$ $=C(z) ; I(f, z)=I(z) ; S(f, z)=S(z)$ pour $z \in \Delta_{0}$. D'après $(R 1)$ de $\S 2$, grâce à la formule de Stokes, on a pour $z \in \Delta_{0}$,

$$
\begin{aligned}
I(z) & =\frac{\sqrt{-1}}{2} \iint_{D(z)} \frac{\partial u(z, w)}{\partial w} d w \wedge \overline{\frac{\partial u(z, w)}{\partial w} d w} \\
& =\frac{\sqrt{-1}}{2} \int_{C(z)} u(z, w) \frac{\overline{\partial u(z, w)}}{\partial w} d w .
\end{aligned}
$$

On fixe un point $\zeta_{k}$ sur chaque composante frontière $C_{k}\left(z_{0}\right)(k=1, \cdots, q)$ de $D\left(z_{0}\right): C\left(z_{0}\right)=\bigcup_{k=1}^{q} C_{k}\left(z_{0}\right)$. Pour $z \in \Delta_{0}$ on prend le point $\xi_{0}\left(z, \zeta_{k}\right)$ sur la composante frontière $C_{k}(z)$ de $D(z), \xi_{0}(z, \zeta)$ étant définie par $(R 3)$. Il existe un voisinage de $C_{k}(z)$ dans $U(z)$ tel qu'on y détermine uniquement la fonction harmonique conjuguée (multiforme par rapport à $w \in U(z)) u^{*}(z, w)$ de $u(z, w)$ telle que $u^{*}\left(z, \xi_{0}\left(z, \zeta_{k}\right)\right)=0$. D'après $(\partial u(z, w) / \partial w) d w=(1 / 2)(d u(z, w)+$ $\left.\sqrt{-1} d u^{*}(z, w)\right)$ où $d=(\partial / \partial w) d w+(\partial / \partial \bar{w}) d \bar{w}$, il s'ensuit que

$$
\begin{aligned}
& I(z)=\frac{1}{2} S(z)+\frac{1}{4} J(z) ; \\
& J(z)=\int_{C(z)} u(z, w) \overline{d u^{*}(z, w) .}
\end{aligned}
$$


Par définition de l'intégrale de Stieltjes et (2.1), il vient pour $z \in \Delta_{0}$

$$
\begin{aligned}
J(z) & =\int_{C\left(z_{0}\right)} u\left(z, \xi_{0}(z, \zeta)\right) \overline{d u^{*}\left(z, \xi_{0}(z, \zeta)\right)} \\
& =\int_{C\left(z_{0}\right)} f_{0}(z, \zeta) \overline{d u^{*}\left(z, \xi_{0}(z, \zeta)\right)} .
\end{aligned}
$$

On considère la transformation $\tau: z \rightarrow t=z-z_{0}$ de $\Delta_{0}$ sur le polydisque $\tilde{\Delta}_{0}$ de centre l'origine de l'espace $\left(t_{1}, \cdots, t_{n}\right) . \quad \operatorname{Par}(z, w) \rightarrow(\tau(z), w), D(\Subset U)$ se transforme $\tilde{D}(\Subset \tilde{U})$ qui est l'ouvert connexe étalé au-dessus de $\tilde{\Delta}_{0} \times C$. On a $D\left(z_{0}\right)$ $=\tilde{D}(0)$ et $\partial D\left(z_{0}\right)=C\left(z_{0}\right)=\partial \tilde{D}(0)=\tilde{C}(0)$. Pour simplicité d'écriture, on pose pour $t=\tau(z), f_{0}(z, \zeta)=f(t, \zeta) ; u(z, w)=u(t, w) ; \xi_{0}(z, \zeta)=\xi(t, \zeta) ; J(z)=\widetilde{J}(t)$ et $S(z)$ $=\tilde{S}(t)$. Il en vient pour $i, j=1, \cdots, n$ et $t \in \tilde{\Delta}_{0}, \zeta \in \tilde{C}(0)$

$$
\frac{\partial^{2} f(t, \zeta)}{\partial t_{i} \partial \bar{t}_{j}}=\frac{\partial \xi(t, \zeta)}{\partial \bar{t}_{i}}=0 ; \quad \xi(0, \zeta)=\zeta ; \quad u(t, \zeta)=f(t, \zeta)
$$

On obtient pour $(t, \zeta) \in \tilde{\Delta}_{0} \times \tilde{C}(0)$ les développements:

$$
\begin{aligned}
f(t, \zeta)= & f(0, \zeta)+\sum_{i=1}^{n}\left\{\frac{\partial f}{\partial t_{i}}(0, \zeta) t_{i}+\frac{\partial f}{\partial \bar{t}_{i}}(0, \zeta) \bar{t}_{i}\right\} \\
& +\sum_{i, j=1}^{n}\left\{A_{i j}(\zeta) t_{i} t_{j}+B_{i j}(\zeta) \bar{t}_{i} \bar{t}_{j}\right\}+O\left(|t|^{3}\right) \\
\xi(t, \zeta)=\zeta & +\sum_{i=1}^{n} \frac{\partial \xi}{\partial t_{i}}(0, \zeta) t_{i}+\sum_{i, j=1}^{n} C_{i j}(\zeta) t_{i} t_{j}+O\left(|t|^{3}\right)
\end{aligned}
$$

où $A_{i j}(\zeta), B_{i j}(\zeta), C_{i j}(\zeta)$ ne dépendent pas de $t \in \tilde{\Delta}_{0}$. Soit $\zeta \in \tilde{C}(0)$. Il existe un voisinage de $(0, \zeta)$ dans $\tilde{U}$ dans lequel on a, d'après $(\tilde{T})$ et $\partial^{2} u^{*} / \partial w \partial \bar{w}=0$, le développement

$$
\begin{aligned}
u^{*}(t, \xi(t, \zeta))= & u^{*}(0, \zeta) \\
& +\sum_{i=1}^{n}\left\{\frac{\partial u^{*}}{\partial t_{i}}(0, \zeta) t_{i}+\frac{\partial u^{*}}{\partial \bar{t}_{i}}(0, \zeta) \bar{t}_{i}\right\}+\frac{\partial u^{*}}{\partial w}(0, \zeta)(\xi(t, \zeta)-\zeta) \\
& +\frac{\partial u^{*}}{\partial \bar{w}}(0, \zeta)(\overline{\xi(t, \zeta)-\zeta}) \\
& +\sum_{i, j=1}^{n} \frac{\partial^{2} u^{*}}{\partial t_{i} \partial \bar{t}_{j}}(0, \zeta) t_{i} \bar{t}_{j}+\sum_{j=1}^{n}\left\{\frac{\partial^{2} u^{*}}{\partial \bar{t}_{j} \partial w}(0, \zeta) \bar{t}_{j}(\xi(t, \zeta)-\zeta)\right. \\
& \left.+\frac{\partial^{2} u^{*}}{\partial t_{j} \partial \bar{w}}(0, \zeta) t_{j}(\overline{\xi(t, \zeta)-\zeta})\right\} \\
& +\sum_{i, j=1}^{n}\left\{\alpha_{i j}(\zeta) t_{i} t_{j}+\beta_{i j}(\zeta) \bar{t}_{i} \bar{t}_{j}+\gamma_{i j}(\zeta)(\xi(t, \zeta)-\zeta)^{2}+\delta_{i j}(\zeta)\left(\overline{\left.\xi(t, \zeta)-\zeta)^{2}\right\}}\right.\right. \\
& +O\left((|t|+\mid \xi(t, \zeta)-\zeta)^{3}\right)
\end{aligned}
$$


où $\alpha_{i j}(\zeta), \beta_{i j}(\zeta), \gamma_{i j}(\zeta), \delta_{i j}(\zeta)$ ne dépendent pas de $t \in \tilde{\Delta}_{0}$. On y substitue l'expression (6.3') et obtient

$$
\begin{aligned}
u^{*}(t, \xi(t, \zeta))= & u^{*}(0, \zeta) \\
& +\sum_{i=1}^{n}\left[\left\{\frac{\partial u^{*}}{\partial t_{i}}+\frac{\partial u^{*}}{\partial w} \frac{\partial \xi}{\partial t_{i}}\right\}_{(0, \zeta)} t_{i}+\left\{\frac{\partial u^{*}}{\partial \bar{t}_{i}}+\frac{\partial u^{*}}{\partial \bar{w}} \overline{\left.\left.\left(\frac{\partial \xi}{\partial t_{i}}\right)\right\}_{(0, \zeta)} \bar{t}_{i}\right]}\right.\right. \\
& +\sum_{i, j=1}^{n}\left\{\frac{\partial^{2} u^{*}}{\partial t_{i} \partial \bar{t}_{j}}+\frac{\partial^{2} u^{*}}{\partial \bar{t}_{j} \partial w} \frac{\partial \xi}{\partial t_{i}}+\frac{\partial^{2} u^{*}}{\partial t_{i} \partial \bar{w}} \overline{\left(\frac{\partial \xi}{\partial t_{j}}\right)}\right\}_{(0, \zeta)} t_{i} \bar{t}_{j} \\
& +\sum_{i, j=1}^{n}\left(a_{i j}(\zeta) t_{i} t_{j}+b_{i j}(\zeta) \bar{t}_{i} \bar{t}_{j}\right)+O\left(|t|^{3}\right),
\end{aligned}
$$

où $a_{i j}(\zeta), b_{i j}(\zeta)$ ne dépendent pas de $t \in \tilde{\Delta}_{0}$. Il s'ensuit par (6.3) que $J(z)=\tilde{J}(t)$ se développe en série de la puissance de $t$ :

$$
\begin{aligned}
\tilde{J}(t)= & \int_{\tilde{c}(0)} f(t, \zeta) \overline{d u^{*}(t, \xi(t, \zeta))} \\
= & \int_{\tilde{c}(0)} f(0, \zeta) \overline{d u^{*}(0, \zeta)} \\
& +\sum_{i=1}^{n} t_{i} \int_{\tilde{\sigma}(0)} f(0, \zeta) d\left(\frac{\partial \bar{u}^{*}}{\partial t_{i}}+\frac{\partial \bar{u}^{*}}{\partial w} \frac{\partial \xi}{\partial t_{i}}\right)(0, \zeta)+\frac{\partial f}{\partial t_{i}}(0, \zeta) d \overline{u^{*}(0, \zeta)} \\
& +\sum_{i=1}^{n} \bar{t}_{i} \int_{\tilde{c}(0)} f(0, \zeta) d\left(\overline{\left.\frac{\partial u^{*}}{\partial t_{i}}+\frac{\partial u^{*}}{\partial w} \frac{\partial \xi}{\partial t_{i}}\right)(0, \zeta)+\frac{\partial f}{\partial \bar{t}_{i}}(0, \zeta) d \overline{u^{*}(0, \zeta)}}\right. \\
& +\sum_{i, j=1}^{n} t_{i} \bar{t}_{j} \int_{\tilde{c}(0)}\left\{\begin{array}{c}
f(0, \zeta) d\left(\frac{\partial^{2} \bar{u}^{*}}{\partial t_{i} \partial \bar{t}_{j}}+\frac{\partial^{2} \bar{u}^{*}}{\partial t_{i} \partial \bar{w}}\left(\frac{\partial \xi}{\partial t_{j}}\right)+\frac{\partial^{2} \bar{u}^{*}}{\partial \bar{t}_{j} \partial w} \frac{\partial \xi}{\partial t_{i}}\right)(0, \zeta) d\left(\frac{\partial u^{*}}{\partial t_{j}}+\frac{\partial u^{*}}{\partial w} \frac{\partial \xi}{\partial t_{j}}\right)(0, \zeta) \\
\quad+\frac{\partial f}{\partial \bar{t}_{j}}(0, \zeta) d\left(\frac{\partial \bar{u}^{*}}{\partial t_{i}}+\frac{\partial \bar{u}^{*}}{\partial w} \frac{\partial \xi}{\partial t_{i}}\right)(0, \zeta)
\end{array}\right. \\
& +\sum_{i, j=1}^{n}\left(K_{i j} t_{i} t_{j}+L_{i j} \bar{t}_{i} \bar{t}_{j}\right)+O\left(|t|^{3}\right),
\end{aligned}
$$

où $K_{i j}, L_{i j}$ sont constantes. On a donc

$$
\begin{aligned}
& \frac{\partial^{2} J}{\partial z_{i} \partial \bar{z}_{j}}\left(z_{0}\right)=\frac{\partial^{2} \tilde{J}}{\partial t_{i} \partial \bar{t}_{j}}(0)=J_{1}+J_{2}+J_{3}+J_{4} ; \\
& J_{1}=\int_{\tilde{\sigma}(0)} f(0, \zeta) d\left(\frac{\partial \bar{u}^{*}}{\partial t_{i} \partial \bar{t}_{j}}\right) ; \\
& J_{2}=\int_{\tilde{c}(0)} f(0, \zeta) d\left(\frac{\partial^{2} \bar{u}^{*}}{\partial t_{i} \partial \bar{w}}\left(\overline{\frac{\partial \xi}{\partial t_{j}}}\right)+\frac{\partial^{2} \bar{u}^{*}}{\partial \bar{t}_{j} \partial w} \frac{\partial \xi}{\partial t_{i}}\right)(0, \zeta) ; \\
& J_{3}=\int_{\tilde{c}(0)} \frac{\partial f}{\partial t_{i}}(0, \zeta) d\left(\overline{\left.\frac{\partial u^{*}}{\partial t_{j}}+\frac{\partial u^{*}}{\partial w} \frac{\partial \xi}{\partial t_{j}}\right)(0, \zeta) ;}\right.
\end{aligned}
$$




$$
J_{4}=\int_{\tilde{c}(0)} \frac{\partial f}{\partial \bar{t}_{j}}(0, \zeta) d\left(\frac{\partial \bar{u}^{*}}{\partial t_{i}}+\frac{\partial \bar{u}^{*}}{\partial w} \frac{\partial \xi}{\partial t_{i}}\right)(0, \zeta) .
$$

D'après $\partial \bar{u}^{*} / \partial w=-\sqrt{-1} \partial \bar{u} / \partial w$ et $\partial \bar{u}^{*} / \partial \bar{w}=\sqrt{-1} \partial \bar{u} / \partial \bar{w}$, on a dans un voisinage de $\tilde{C}(0)$ dans $\tilde{U}, \partial^{2} \bar{u}^{*} / \partial t_{i} \partial \bar{w}=\sqrt{-1} \partial^{2} \bar{u} / \partial t_{i} \partial \bar{w}$ et $\partial^{2} \bar{u}^{*} / \partial \bar{t}_{j} \partial w=-\sqrt{-1} \partial^{2} \bar{u} / \partial \bar{t}_{j} \partial w$, d'où elles sont uniformes pour $w \in \tilde{C}(0)$. Il en vient

$$
J_{2}=-\sqrt{-1} \int_{\tilde{\sigma}(0)}\left(\frac{\partial^{2} \bar{u}}{\partial t_{i} \partial \bar{w}}\left(\overline{\frac{\partial \xi}{\partial t_{j}}}\right)-\frac{\partial^{2} \bar{u}}{\partial \bar{t}_{j} \partial w} \frac{\partial \xi}{\partial t_{i}}\right)(0, \zeta) d f(0, \zeta)
$$

D'après $(\tilde{T}): f(0, \zeta)=u(0, \zeta)$ pour $\zeta \in \tilde{C}(0)$, on a $d f(0, w)=(\partial u / \partial w)(0, w) d w+$ $(\partial u / \partial \bar{w})(0, w) d \bar{w}$ pour $w \in \tilde{C}(0)$. D'après $d u^{*}=-\sqrt{-1}((\partial u / \partial w) d w-(\partial u / \partial \bar{w}) d \bar{w})$, on a $d f(0, w)=\sqrt{-1} d u^{*}(0, w)+2(\partial u / \partial \bar{w})(0, w) d \bar{w}=-\sqrt{-1} d u^{*}(0, w)+$ $2(\partial u / \partial w)(0, w) d w$ le long de $\tilde{C}(0)$. Il vient

$$
\begin{aligned}
& J_{2}=-\sqrt{-1} \int_{\bar{c}(0)}\left\{\begin{array}{c}
\left(\frac{\partial^{2} \bar{u}}{\partial t_{i} \partial \bar{w}}\left(\overline{\frac{\partial \xi}{\partial t_{j}}}\right)\right)(0, w)\left(\sqrt{-1} d u^{*}(0, w)+2 \frac{\partial u}{\partial \bar{w}}(0, w) d \bar{w}\right) \\
+\left(\frac{\partial^{2} \bar{u}}{\partial \bar{t}_{j} \partial w} \frac{\partial \xi}{\partial t_{i}}\right)(0, w)\left(\sqrt{-1} d u^{*}(0, w)-2 \frac{\partial u}{\partial w}(0, w) d w\right)
\end{array}\right\} \\
& =\int_{\tilde{c}(0)}\left\{\frac{\partial^{2} \bar{u}}{\partial t_{i} \partial \bar{w}} \overline{\left(\frac{\partial \xi}{\partial t_{j}}\right)}+\frac{\partial^{2} \bar{u}}{\partial \bar{t}_{j} \partial w} \frac{\partial \xi}{\partial t_{i}}\right\}_{(0, w)} d u^{*}(0, w) \\
& -2 \sqrt{-1} \int_{\tilde{c}(0)}\left\{\frac{\partial^{2} \bar{u}}{\partial t_{i} \partial \bar{w}}\left(\frac{\overline{\partial \xi}}{\partial t_{j}}\right) \frac{\partial u}{\partial \bar{w}}\right\}_{(0, w)} d \bar{w}-\left\{\frac{\partial^{2} \bar{u}}{\partial \bar{t}_{j} \partial w} \frac{\partial \xi}{\partial t_{i}} \frac{\partial u}{\partial w}\right\}_{(0, w)} d w .
\end{aligned}
$$

D’aprés (2.6), (2.3) et (2.4), il vient

$$
\begin{aligned}
& J_{2}=J_{21}+J_{22}+J_{23} ; \\
& J_{21}=-\int_{\tilde{c}(0)} \frac{\partial^{2} \bar{u}}{\partial t_{i} \partial \bar{t}_{j}}(0, w) d u^{*}(0, w) \\
& J_{22}=2 \sqrt{-1} \int_{\tilde{c}(0)}\left\{\frac{\partial^{2} \bar{u}}{\partial t_{i} \partial \bar{w}} \frac{\partial u}{\partial \bar{t}_{j}}\right\}_{(0, w)} d \bar{w}-\left\{\frac{\partial^{2} \bar{u}}{\partial \bar{t}_{j} \partial w} \frac{\partial u}{\partial t_{i}}\right\}_{(0, w)} d w \\
& J_{23}=-2 \sqrt{-1} \int_{\tilde{c}(0)}\left\{\frac{\partial^{2} \bar{u}}{\partial t_{i} \partial \bar{w}} \frac{\partial f}{\partial \bar{t}_{j}}\right\}_{(0, w)} d \bar{w}-\left\{\frac{\partial^{2} \bar{u}}{\partial \bar{t}_{j} \partial w} \frac{\partial f}{\partial t_{i}}\right\}_{(0, w)} d w
\end{aligned}
$$

D'après $\partial u^{*} / \partial w=-\sqrt{-1} \partial u / \partial w$ et (2.3), on a

$$
J_{3}=\int_{\tilde{\sigma}(0)} \frac{\partial f}{\partial t_{i}}(0, \zeta) d \overline{\left(\frac{\partial u^{*}}{\partial t_{j}}-\sqrt{-1}\left(\frac{\partial f}{\partial t_{j}}-\frac{\partial u}{\partial t_{j}}\right)\right)(0, \zeta)}
$$

Pusique $d \overline{\left(\partial u^{*} / \partial t_{j}+\sqrt{-1} \partial u / \partial t_{j}\right)}=-2 \sqrt{-1}\left(\partial^{2} \bar{u} / \partial \bar{t}_{j} \partial w\right) d w$, on a

$$
J_{3}=\sqrt{-1} \int_{\tilde{c}(0)} \frac{\partial f}{\partial t_{i}}(0, \zeta) d \overline{\frac{\partial f}{\partial t_{j}}(0, \zeta)}-2 \sqrt{-1} \int_{\tilde{c}(0)}\left\{\frac{\partial^{2} \bar{u}}{\partial \bar{t}_{j} \partial w} \frac{\partial f}{\partial t_{i}}\right\}_{(0, w)} d w .
$$


On a pareillement,

$$
J_{4}=-\sqrt{-1} \int_{\tilde{c}(0)} \frac{\partial f}{\partial \bar{t}_{j}}(0, \zeta) d \frac{\partial \bar{f}}{\partial t_{i}}(0, \zeta)+2 \sqrt{-1} \int_{\tilde{c}(0)}\left\{\frac{\partial^{2} \bar{u}}{\partial t_{i} \partial \bar{w}} \frac{\partial f}{\partial \bar{t}_{j}}\right\}_{(0, w)} d \bar{w}
$$

Le second membre de cette expression de $J_{3}$ (resp. $J_{4}$ ) est égale à moins du second terme (resp. premier terme) de $J_{23}$. Il s'ensuit que

$$
\begin{gathered}
\frac{\partial^{2} J}{\partial z_{i} \partial \bar{z}_{j}}\left(z_{0}\right)=\left(J_{1}+J_{21}\right)+J_{22}+\left(J_{23}+J_{3}+J_{4}\right) \\
=K_{1}+K_{2}+K_{3} ; \\
K_{1}=\int_{\tilde{c}(0)} f(0, w) d\left(\frac{\partial^{2} \bar{u}^{*}}{\partial t_{i} \partial \bar{t}_{j}}\right)(0, w)-\frac{\partial^{2} \bar{u}}{\partial t_{i} \partial \bar{t}_{j}}(0, w) d u^{*}(0, w) ; \\
K_{2}=2 \sqrt{-1} \int_{\tilde{c}(0)}\left\{\frac{\partial^{2} \bar{u}}{\partial t_{i} \partial \bar{w}} \frac{\partial u}{\partial \bar{t}_{j}}\right\}_{(0, w)} d \bar{w}-\left\{\frac{\partial^{2} \bar{u}}{\partial \bar{t}_{j} \partial w} \frac{\partial u}{\partial t_{i}}\right\}_{(0, w)} d w ; \\
K_{3}=\sqrt{-1} \int_{\tilde{\sigma}(0)} \frac{\partial f}{\partial t_{i}}(0, w) d \frac{\partial f}{\partial t_{j}}(0, w)-\frac{\partial f}{\partial \bar{t}_{j}}(0, w) d \frac{\partial \bar{f}}{\partial t_{i}}(0, w) .
\end{gathered}
$$

D'après $f(0, \zeta)=u(0, \zeta)$ sur $\tilde{C}(0)$, il vient

$$
\begin{aligned}
K_{1} & =\int_{\tilde{\sigma}(0)} u(0, w) d \frac{\partial^{2} \bar{u}^{*}}{\partial t_{i} \partial \bar{t}_{j}}(0, w)-\frac{\partial^{2} \bar{u}}{\partial t_{i} \partial \bar{t}_{j}}(0, w) d u^{*}(0, w) \\
& =\left\{\frac{\partial^{2}}{\partial t_{i} \partial \bar{t}_{j}}\left(\int_{\tilde{c}(0)} u(0, w) d \bar{u}^{*}(t, w)-\bar{u}(t, w) d u^{*}(0, w)\right)\right\}_{t=0}
\end{aligned}
$$

Puisque $u(t, w)$ et $u(0, w)$ sont harmoniques par rapport à $w$ dans un voisinage de $\tilde{D}(0)$ dans $\tilde{U}(0)$, il en résulte gràce à la formule de Stokes que l'intégrale dans la ( ) est toujours nulle pour chaque $t \in \tilde{\Delta}_{0}$, d'où $K_{1}=0$. On a pareillement

$$
K_{2}=4 \iint_{\tilde{D}(0)}\left\{\frac{\partial^{2} \bar{u}}{\partial t_{i} \partial \bar{w}} \frac{\partial^{2} u}{\partial \bar{t}_{j} \partial w}+\frac{\partial^{2} u}{\partial t_{i} \partial \bar{w}} \frac{\partial^{2} \bar{u}}{\partial \bar{t}_{j} \partial w}\right\}_{(0, w)} d u d v
$$

En autre côté, par $S(z)=\tilde{S}(t)$ pour $z-z_{0}=t$ et $(\tilde{T})$, il vient

$$
\begin{aligned}
\frac{\partial^{2} S}{\partial z_{i} \partial \bar{z}_{j}}\left(z_{0}\right) & =\frac{\partial^{2} \tilde{S}}{\partial t_{i} \partial \bar{t}_{j}}(0) \\
& =\frac{\sqrt{-1}}{2}\left\{\frac{\partial^{2}}{\partial t_{i} \partial \bar{t}_{j}} \int_{\tilde{c}(0)} f(t, \zeta) d \overline{f(t, \zeta)}\right\}_{t=0} \\
& =\frac{\sqrt{-1}}{2} \int_{\tilde{c}(0)} \frac{\partial f}{\partial t_{i}}(0, \zeta) d \overline{\frac{\partial f}{\partial t_{j}}(0, \zeta)}+\frac{\partial f}{\partial \bar{t}_{j}}(0, \zeta) d \frac{\partial \bar{f}^{\prime}}{\partial t_{i}}(0, \zeta) .
\end{aligned}
$$

On a enfin d'après (6.2) 


$$
\begin{aligned}
\frac{\partial^{2} I}{\partial z_{i} \partial \bar{z}_{j}}\left(z_{0}\right)= & \frac{1}{2} \frac{\partial^{2} \tilde{S}}{\partial t_{i} \partial \bar{t}_{j}}(0)+\frac{1}{4}\left(K_{2}+K_{3}\right) \\
= & \frac{\sqrt{-1}}{2} \int_{\tilde{c}(0)} \frac{\partial f}{\partial t_{i}}(0, \zeta) d \overline{\frac{\partial f}{\partial t_{j}}(0, \zeta)} \\
& +\iint_{\tilde{D}(0)}\left\{\frac{\partial^{2} \bar{u}}{\partial t_{i} \partial \bar{w}} \frac{\partial^{2} u}{\partial \bar{t}_{j} \partial w}+\frac{\partial^{2} u}{\partial t_{i} \partial \bar{w}} \frac{\partial^{2} \bar{u}}{\partial \bar{t}_{j} \partial w}\right\}_{(0, w)} d u d v
\end{aligned}
$$

D'après $z_{i}-z_{0 i}=t_{i}(i=1, \cdots, n)$, cette égalité n'est autre que celle de la formule 4 pour $z=z_{0}$. $z_{0}$ étant quelconque de $\Delta$, la formule 4 est démontrée.

D'après (6.4) on a, par le même calcul que $J_{4}$

$$
\begin{aligned}
\frac{\partial \tilde{J}}{\partial t_{i}}(0)= & \int_{\tilde{c}(0)} f(0, \zeta) d\left\{\frac{\partial \bar{u}^{*}}{\partial t_{i}}+\frac{\partial \bar{u}^{*}}{\partial w} \frac{\partial \xi}{\partial t_{i}}\right\}(0, \zeta)+\frac{\partial f}{\partial t_{i}} d \bar{u}^{*}(0, \zeta) \\
= & \int_{\tilde{c}(0)} \frac{\partial f}{\partial t_{i}}(0, \zeta) d \bar{u}^{*}(0, \zeta)-\sqrt{-1} f(0, \zeta) d \frac{\partial \bar{f}_{\partial t_{i}}(0, \zeta)}{} \\
& +4 \iint_{D(0)}\left\{\frac{\partial u}{\partial w} \frac{\partial^{2} \bar{u}}{\partial t_{i} \partial \bar{w}}\right\}_{(0, w)} d u d v .
\end{aligned}
$$

D’après $(6.2)$ et $(\tilde{T})$, on a aisément

$$
\begin{aligned}
\frac{\partial I}{\partial z_{i}}\left(z_{0}\right) & =\frac{\sqrt{-1}}{4} \int_{\tilde{\sigma}(0)} \frac{\partial f_{i}}{\partial t_{i}}(0, \zeta) d \overline{\left(u+\sqrt{-1} u^{*}\right)(0, \zeta)}+\iint_{\tilde{D}(0)}\left\{\frac{\partial u}{\partial w} \frac{\partial^{2} \bar{u}}{\partial t_{i} \partial \bar{w}}\right\}_{(0, w)} d u d v \\
& =\iint_{\tilde{D}(0)}\left\{\frac{\partial u_{1}}{\partial w} \frac{\partial \bar{u}}{\partial \bar{w}}+\frac{\partial^{2} \bar{u}}{\partial t_{i} \partial \bar{w}} \frac{\partial u}{\partial w}\right\}_{(0, w)} d u d v,
\end{aligned}
$$

où $u_{1}(t, w)\left(=H_{\partial f / \partial z i}(z, w)\right)$ désigne la solution du problème de Dirichlet sur $\mathfrak{D}(z)$ avec fonction frontière $\partial f / \partial z_{i}$ sur $\partial \mathfrak{D}$. On a donc la formule suivante:

$$
\frac{\partial I(f, z)}{\partial z_{i}}=\iint_{D(z)}\left\{\frac{\partial H_{\partial f / \partial z_{i}}}{\partial w} \frac{\partial \bar{H}_{f}}{\partial \bar{w}}+\frac{\partial^{2} \bar{H}_{f}}{\partial z_{i} \partial \bar{w}} \frac{\partial H_{f}}{\partial w}\right\} d u d v
$$

On distingue trois cas particuliers pour $f$ dans la formule 4. Pour cela, soit $\Xi$ la section analytique quelconque de $\hat{\mathfrak{D}}$ sur $\Delta$ telle que $E(\Delta) \subset \partial \mathfrak{D}$. Pour la fonction $f$ sur $\partial \mathfrak{D}$, on écrit $f \mid \Xi$ la restriction de $f$ sur la surface analytique $\Xi(\Delta)$ de $\hat{D}$.

$1^{\text {er }}$ cas: $f \mid \xi$ est une constante réelle pour $z \in \Delta$ dépendant de $E$. Alors la formule 4 devient d'après $\partial f / \partial z_{i}=0$

$$
\frac{\partial^{2} I(f, z)}{\partial z_{i} \partial \bar{z}_{j}}=2 \iint_{D(z)} \frac{\partial^{2} H_{f}}{\partial z_{i} \partial \bar{w}} \frac{\partial^{2} H_{f}}{\partial \bar{z}_{j} \partial w} d u d v
$$

d'où la matrice $\left\{\partial^{2} I(f, z) / \partial z_{i} \partial \bar{z}_{j}\right\}_{i, j=1, \cdots, n}$ pour $z \in \Delta$ est non-négative définitive sur le corps complexe. On a de plus, d'après (6.5), 


$$
\frac{\partial I(f, z)}{\partial z_{i}}=\iint_{D(z)} \frac{\partial^{2} H_{f}}{\partial z_{i} \partial \bar{w}} \frac{\partial H_{f}}{\partial w} d u d v
$$

Il s'ensuit que, pour nombres complexes quelconques $z_{1}, \cdots, z_{n}$,

$$
\begin{aligned}
\sum_{i, j=1}^{n} z_{i} \bar{z}_{j} \frac{\partial^{2}}{\partial z_{i} \partial \bar{z}_{j}}\left(\frac{1}{I(f, z)}\right) & \\
= & \frac{-1}{I(f, z)^{3}}\left\{\|\omega\|_{\triangleright(z)}^{2} \cdot\left\|\sum_{i=1}^{n} z_{i} \frac{\partial \omega}{\partial \bar{z}_{i}}\right\|_{\triangleright(z)}^{2}-\left|\left(\omega, \sum_{i=1}^{n} z_{i} \frac{\partial \omega}{\partial \bar{z}_{i}}\right)\right|^{2}\right\}
\end{aligned}
$$

où $\omega=\omega(z, w)=\left(\partial H_{f} / \partial w\right) d w$. Grâce à l'inégalité de Schwarz, la \{ \} est nonnégative. On en conclut que pour toute fonction frontière $f(\zeta) d u 1^{\text {er }}$ cas, la réciproque de $I(f, z)$ est positive et pluri-surharmonique dans $\Delta$.

$2^{\text {ième }}$ cas: $f \mid \Xi$ est analytique pour $z \in \Delta$. Alors la formule 4 et l'expression (6.5) entraînent

$$
\begin{gathered}
\frac{\partial I(\bar{f}, z)}{\partial z_{i}}=\iint_{\triangleright(z)} \frac{\partial^{2} H_{f}}{\partial z_{i} \partial \bar{w}} \frac{\partial H_{\mathcal{J}}}{\partial w} d u d v \\
\frac{\partial^{2} I(\bar{f}, z)}{\partial z_{i} \partial \bar{z}_{j}}=\iint_{\triangleright(z)}\left(\frac{\partial^{2} H_{f}}{\partial z_{i} \partial \bar{w}} \frac{\partial^{2} H_{\bar{f}}}{\partial \bar{z}_{j} \partial w}+\frac{\partial^{2} H_{\vec{f}}}{\partial z_{i} \partial \bar{w}} \frac{\partial^{2} H_{f}}{\partial \bar{z}_{j} \partial w}\right) d u d v
\end{gathered}
$$

d'où la matrice $\left\{\operatorname{Re}\left(\partial^{2} I(\bar{f}, z) / \partial z_{i} \partial \bar{z}_{j}\right)\right\}_{i, j=1, \ldots, n}$ pour $z \in \Delta$ est non-négative définitive sur le corps réel.

$3^{\text {ième }}$ cas: $\quad n=1$ et $f \mid \Xi$ est analytique pour $z \in \Delta$. La formule 4 devient celle suivante comparable à celle $(6.1)$

$$
\frac{\partial^{2} I(f, z)}{\partial z \partial \bar{z}}=\frac{\partial^{2} S(f, z)}{\partial z \partial \bar{z}}+\iint_{\circledast(z)}\left(\left|\frac{\partial^{2} H_{f}}{\partial \bar{z} \partial w}\right|^{2}+\left|\frac{\partial^{2} H_{\mathcal{f}}}{\partial \bar{z} \partial w}\right|^{2}\right) d u d v
$$

On en conclut que pour toute fonction frontière $f d u 3^{i e ̀ m e}$ cas, il vient $\partial^{2} I(f, z) / \partial z \partial \bar{z} \geqq \partial^{2} S(f, z) / \partial z \partial \bar{z}$ dans $\Delta$, et de plus que sous l'hypothèse que la restriction de $H_{f}$ sur $\mathfrak{D}(0)$ soit analytique, l'égalité est valable dans 4 , si et seulement si $H_{f}$ est analytique dans tout $\mathfrak{S}$.

En effet, supposons que l'égalité soit valable dans $\Delta$. On abrège $H_{f}(z, w)$ $=u(z, w)$. Soit $P_{0} \in \partial \mathfrak{D}(0)$. Prenons d'après la remarque 2 , des $\pi$-coordonnées d'un voisinage $\mathscr{V}$ de $P_{0}$ dans $\hat{\mathfrak{S}}$ de la forme $(z, w) \in \Delta_{0} \times \delta$ où $\Delta_{0}=\left\{|z|<\rho_{0}\right\}$ et $\delta=\{|\boldsymbol{w}|<1\}$ telles que $\mathscr{V} \cap \partial \mathfrak{D}$ corresponde à $\Delta_{0} \times \gamma$ où $\gamma=\{\boldsymbol{w} \in \delta: \operatorname{Im} w=0\}$. Il vient par (6.8) $\partial^{2} u / \partial \bar{z} \partial w=\partial^{2} \bar{u} / \partial \bar{z} \partial w=0$ dans $\Delta_{0} \times \delta$, d'où $\partial \bar{u} / \partial z$ est, pour $z$ fixé dans $\Delta_{0}$, analytique pour $\boldsymbol{w} \in \delta$. Par notation $(* *)$ de $\S 2$, le $3^{\text {ième }}$ cas entraîne que $f_{0}\left(z, w_{0}\right)$ est, pour $\boldsymbol{w}_{0}$ fixé sur $\gamma$, analytique pour $z \in \Delta_{0} . \quad \operatorname{Par}(H 1)$ de $\S 2$, on a $u\left(z, \boldsymbol{w}_{0}\right)=f_{0}\left(z, \boldsymbol{w}_{0}\right)$ dans $\Delta_{0} \times \gamma$, d'où $\partial \bar{u}\left(z, \boldsymbol{w}_{0}\right) / \partial z=0$. Alors le théorème d'identité concernant les fonctions holomorphes dans $\delta$ entraîne que $(\partial \bar{u} / \partial z)\left(z_{0}, w\right)$ est nulle pour $w \in \delta$, d'où que $u(z, w)$ est, pour $w$ fixé dans $\delta$, analytique pour 
$z \in \Delta_{0}$. On en a le développement $u(z, w)=u(0, w)+\sum_{n=1}^{\infty} A_{n}(w) z^{n}$. D'après $\partial^{2} u / \partial z \partial \bar{w}=0$ dans $\Delta_{0} \times \delta$, tout $A_{n}(w)(n=1,2, \cdots)$ est analytique dans $\delta$. Avec notre hypothèse pour $u(0, w)$, on sait que $u(z, w)$ est analytique dans $\Delta_{0} \times \delta$. Par le prolongement analytique, $u(z, w)$ l'est aussi dans $\mathfrak{D}$. La réciproque est évidemment vraie.

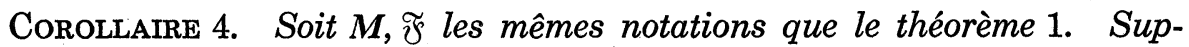
posons que $M$ soit simplement connexe et de type parabolique (voir p. 142 de $Y$. Kusunoki [5]), c'est-à-dire, il n'existe aucune fonction positive et plurisurharmonique non-constante sur $M$. Alors, la famille holomorphe $\mathfrak{F}$ est équivalente à celle triviale.

Preuve. Par la notation à la fin de $\S 1$ pour $z_{0}=0$, on a pour $z^{(k)} \in \Delta_{k}$ $(k=1,2, \cdots), \partial \mathfrak{D}_{k}\left(z^{(k)}\right)=\left\{\Xi_{k}\left(Q_{k}, z^{(k)}\right): Q_{k} \in \partial \mathfrak{D}_{k}(0)\right\}$. On écrit $\delta_{k}$ l'ouvert de $M$ correspondant à $\Delta_{k}$. Supposons que $\delta_{k} \cap \delta_{l} \neq \phi$. Puisque $\mathfrak{\mho}$ est holomorphe, d'après (3) de $\S 1$ il existe uniquement une transformation de $\partial \mathfrak{D}_{k}(0)$ sur $\partial \mathfrak{S}_{l}(0): \mathscr{Q}_{k} \rightarrow \mathscr{Q}_{l}$ telle que $\Xi_{k}\left(Q_{k}, z^{(k)}\right)$ et $\Xi_{l}\left(Q_{l}, z^{(l)}\right)$ s'expriment le même point de $\partial \widetilde{\zeta}, z^{(k)}$ et $z^{(l)}$ correspondant au même point de $\delta_{k} \cap \delta_{l}$. Puisque $M$ est simplement connexe, chaque $Q_{1}$ de $\partial \mathfrak{D}_{1}(0)$ détermine la section analytique $\boldsymbol{E}\left(Q_{1}, \cdot\right)$ de $\bigcup_{k=1}^{\infty} \hat{\mathfrak{D}}_{k}$ sur toute $M$ passant par $Q_{1}$ telle que $\Xi\left(Q_{1}, M\right) \subset \partial \widetilde{\mho}$. Soit donnée une fonction réelle $f$ définie sur $\partial \mathfrak{D}_{1}(0)$. On forme la solution du problème de Dirichlet $H_{f}(z, w)$ sur chaque fibre $\mathfrak{\mho}(z)$ où $z \in M$ ayant la fonction frontière $f\left(Q_{1}\right)$ pour $\Xi\left(Q_{1}, z\right)$ où $Q_{1} \in \partial \mathfrak{S}(0)$, et note $I_{f}(z)$ son intégrale de Dirichlet sur $\mathfrak{F}(z)$. Le $1^{\text {er }}$ cas entraîne que $1 / I_{f}(z)$ est fonction positive pluri-surharmonique sur $M$. Par parabolicité de $M, I_{f}(z)$ est une constant sur $M$. On considère ici une transformation conforme $F(w)$ de $\mathfrak{D}_{1}(0) \cup \partial \mathfrak{D}_{1}(0)$ sur un ouvert connexe $R$ multivalent étalé au-dessus du plan $W$ sans point de ramification et avec frontières lisses. On pose $F=f_{1}+\sqrt{-1} f_{2}\left(f_{1}, f_{2}\right.$ étant réelles). D'après $I_{F}(z)=$ $I_{f_{1}}(z)+I_{f_{2}}(z)-(1 / 2)\left(I_{f_{1}+f_{2}}(z)-I_{f_{1}-f_{2}}(z)\right)$, il s'ensuit que $I_{F}(z)$ est une constante sur $M$, d'où $\partial^{2} I_{F}\left(z^{(1)}\right) / \partial z_{i}^{(1)} \partial \bar{z}_{i}^{(1)}=0(i=1, \cdots, n)$ pour $z^{(1)} \in \Delta_{1}$. Par $H_{F^{\prime}}(0, w)=$ $F(w)$ sur $\mathfrak{D}_{1}(0)$, le $3^{\text {ième }}$ cas entraîne que $H_{F}$ est analytique dans $\mathfrak{D}_{1}$. Puisque $H_{F}\left(z^{(1)}, \Xi\left(Q_{1}, z^{(1)}\right)\right)=F\left(Q_{1}\right)$ sur $\Delta_{1} \times \partial \mathfrak{D}_{1}(0)$, on a $H_{F}\left(z^{(1)}, \partial \mathfrak{D}_{1}\left(z^{(1)}\right)\right)=\partial R$. D'après $\partial F(w) / \partial w \neq 0$ pour aucun $w \in \mathfrak{D}_{1}(0)$, il s'ensuit que $H_{F}\left(z^{(1)}, \mathfrak{D}_{1}\left(z^{(1)}\right)\right)=R$ pour tout $z^{(1)} \in \Delta_{1}$. Donc la famille est équivalente à celle triviale $M \times R$ par la transformation analytique: $(z, w) \rightarrow\left(z, H_{F}(z, w)\right)$.

\section{§7. Variations de périodes}

Soit $R$ une surface de Riemann à bord $\partial R$. D'après le chapitre $V$ du livre [1], on pose $\Gamma_{h}(R)$ l'ensemble des 1-formes harmoniques $\omega=a(w) d u+$ $b(w) d v$ où $w=u+\sqrt{-1} v(u, v$, réels) sur $R$ telles que 


$$
\|\omega\|_{R}^{2}=\iint_{R} \omega \wedge \bar{\omega}^{*}=\iint_{R}\left(|a|^{2}+|b|^{2}\right) d u d v<\infty
$$

où $\omega^{*}=-b(w) d u+a(w) d v$. Avec produit intérieur

$$
(\omega, \sigma)_{R}=\iint_{R} \omega \wedge \bar{\sigma}^{*}
$$

$\Gamma_{h}(R)$ est un espace hilbertien sur le corps complexe. Etant donnée une courbe fermée $\gamma$ sur $R$, il existe uniquement $\sigma_{r}$ réelle de $\Gamma_{h}(R)$ ayant la propriété $(P 1): \int_{r} \omega=\left(\omega, \sigma_{r}^{*}\right)_{R}$ pour toute $\omega \in \Gamma_{h}(R)$. On appelle $\sigma_{r}$ la différentielle reproduisant de $\gamma$. Soit $\tau$ une courbe fermée sur $R$ ou bien un arc sur $R$ partant d'un point de $\partial R$ et arrivant à l'autre de $\partial R$. Alors les propriétés suivantes sont connues (voir p. 130 de [1]) (P2): $\sigma_{r}$ est nulle le long de $\partial R,(P 3): \int_{\tau} \sigma_{r}=$ $\gamma \times \tau$, un nombre entier. Soit $(p, q)$ le type topologique de $R$, on a $2 p+q-1$ courbes fermées de Jordan $\left\{A_{i}, B_{i}\right\} \cup\left\{C_{j}\right\}(i=1, \cdots, p ; j=1, \cdots, q-1)$ qui font une base homologue de $R$. Dans ce livre, on a considéré le sous-espace $\Gamma_{h 0}(R)$ de $\Gamma_{h}(R)$ engenré par $\left\{\sigma_{A_{i}}, \sigma_{B_{i}}, \sigma_{C_{j}}\right\}$.

Maintenant on retroune au triple $(\mathfrak{S}, \Delta, \pi)$ vérifiant aux conditions $(L)$, (T) de type topologique $(p, q)$. On peut écrire, sur chaque $\mathfrak{D}(z)$ où $z \in \Delta, 2 p$ $+q-1=\chi$ courbes fermées ci-dessus $\left\{A_{i}(z), B_{i}(z), C_{j}(z)\right\}$ qui font une base homologue de $\mathfrak{D}(z)$ et se varient continûment avec $z \in \Delta$. On obtient alors la

Formule 5. Pour $\sigma(z, \cdot) \in \Gamma_{h 0}(\mathfrak{D}(z))$ de la forme

$$
\sigma(z, \cdot)=\sum_{i=1}^{p}\left(\alpha_{i} \sigma_{A_{i}(z)}+\beta_{i} \sigma_{B_{i}(z)}\right)+\sum_{j=1}^{q-1} \gamma_{j} \sigma_{C_{j}(z)}
$$

où $\alpha_{i}, \beta_{i}, \gamma_{j}$ sont nombres réels indépendants de $z \in \Delta$, il vient pour $\Omega(z, \cdot)=$ $\sigma(z, \cdot)+\sqrt{-1} \sigma^{*}(z, \cdot) \operatorname{sur} \mathfrak{D}(z)$

$$
\begin{aligned}
& \frac{\partial}{\partial z_{i}}\|\sigma(z, \cdot)\|_{\Phi(z)}^{2}=\frac{1}{2}\left(\Omega(z, \cdot), \partial \Omega(z, \cdot) / \partial \bar{z}_{i}\right)_{\Phi(z)} ; \\
& \frac{\partial^{2}}{\partial z_{i} \partial \bar{z}_{j}}\|\sigma(z, \cdot)\|_{\Phi(z)}^{2}=\left(\partial \Omega(z, \cdot) / \partial \bar{z}_{j}, \partial \Omega(z, \cdot) / \partial \bar{z}_{i}\right)_{\Phi(z)} \cdot
\end{aligned}
$$

Preuve. Soit $z_{0} \in \Delta$. On prend $\left(D, \Delta_{0}, p\right)$ équivalent à $\left(\mathfrak{D}\left|\Delta_{0}, \Delta_{0}, \pi\right| \Delta_{0}\right)$ comme la preuve de la formule 3 . Prenons un point $\zeta_{0}(z)$ de $C_{q}(z)(\subset \partial D(z))$ de façon que $\zeta_{0}(z)$ se varie continûment avec $z \in \Delta_{0}$. On forme sur $D(z)$ où $z \in \Delta_{0}$ la fonction harmonique multiforme $v(z, w)$ telle que $d v(z, w)=\sigma(z, w)$ et $v\left(z, \zeta_{0}(z)\right)=0$. L'expression (7.1) entraîne d'après $(P 1) ;(P 2) ;(P 3)$ respectivement que (1) $\left(\sigma(z, \cdot)-\sigma\left(z_{0}, \cdot\right), \sigma\left(z_{0}, \cdot\right)\right)_{D\left(z_{0}\right)}=\left(\sigma(z, \cdot), \sigma\left(z_{0}, \cdot\right)\right)_{D\left(z_{0}\right)}-\left\|\sigma\left(z_{0}, \cdot\right)\right\|_{D\left(z_{0}\right)}^{2}=$ $\|\sigma(z, \cdot)\|_{D(z)}^{2}-\left\|\sigma\left(z_{0}, \cdot\right)\right\|_{D\left(z_{0}\right)}^{2} ;(2)$ on trouve une branche de $v(z, w)$ telle que 
$v(z, w)$ soit constante $\gamma_{j}$ (resp. 0 ) indépendante de $z \in \Delta_{0}$ sur la composante frontière $C_{j}(z)$ pour $1 \leqq j \leqq q-1$ (resp. $j=q$ ) de $D(z)$; (3) pour chaque courbe fermée $\tau(z)$ sur $D(z)$ telle que $\tau(z)$ se varie continûment avec $z \in \Delta_{0}$, la période de $v(z, w)$ pour $\tau(z)$ est de la forme $\sum_{i=1}^{p} \alpha_{i}\left(A_{i}(z) \times \tau(z)\right)+\beta_{i}\left(B_{i}(z) \times \tau(z)\right)$, qui n'en dépend pas de $z \in \Delta_{0} . \quad v(z, w)-v\left(z_{0}, w\right)$ étant, d'après (3), uniforme sur $D\left(z_{0}\right)$, la formule de Stokes entraîne par (1) que pour $z \in \Delta_{0}$

$$
\int_{C\left(z_{0}\right)}\left(v(z, w)-v\left(z_{0} w\right)\right) \sigma^{*}\left(z_{0}, w\right)=\|\sigma(z, \cdot)\|_{D(z)}^{2}-\left\|\sigma\left(z_{0}, \cdot\right)\right\|_{D(z)}^{2} \cdot
$$

Il s'ensuit par $z_{i} \bar{z}_{j}$-différentielle que

$$
\int_{C\left(z_{0}\right)} \frac{\partial^{2} v(z, w)}{\partial z_{i} \partial \bar{z}_{j}} \sigma^{*}\left(z_{0}, w\right)=\frac{\partial^{2}}{\partial z_{i} \partial \bar{z}_{j}}\|\sigma(z, \cdot)\|_{D(z)}^{2} \cdot
$$

D'après (2) on applique l'expression (2.7) et obtient d'après $\sigma^{*}\left(z_{0}, w\right)=-2 \sqrt{-1}$ $(\partial v(z, w) / \partial w) d w=2 \sqrt{-1}(\partial v(z, w) / \partial \bar{w}) d \bar{w}$ le long de $C\left(z_{0}\right)$

$$
\begin{aligned}
& \left\{\frac{\partial^{2}}{\partial z_{i} \partial \bar{z}_{j}}\|\sigma(z, \cdot)\|_{D(z)}^{2}\right\}_{z=z_{0}} \\
& \quad=2 \sqrt{-1} \int_{C\left(z_{0}\right)}\left\{\frac{\partial^{2} v}{\partial z_{i} \partial \bar{w}} \frac{\partial v}{\partial \bar{z}_{\jmath}}\right\}_{\left(z_{0}, w\right)} d \bar{w}-\left\{\frac{\partial^{2} v}{\partial \bar{z}_{j} \partial w} \frac{\partial v}{\partial z_{i}}\right\}_{\left(z_{0}, w\right)} d w .
\end{aligned}
$$

Puisque toutes les fonctions sous l'intégrale sont, par (3), uniformes et harmonique sur $D\left(z_{0}\right)$, il vient

$$
\begin{aligned}
& =4 \sqrt{-1} \iint_{D\left(z_{0}\right)}\left\{\frac{\partial^{2} v}{\partial z_{i} \partial \bar{w}} \frac{\partial^{2} v}{\partial \bar{z}_{j} \partial w}\right\}_{\left(z_{0}, w\right)} d w \wedge d \bar{w} \\
& =\left\{\left(\frac{\partial \Omega(z, \cdot)}{\partial \bar{z}_{j}} \frac{\partial \Omega(z, \cdot)}{\partial \bar{z}_{i}}\right)_{D(z)}\right\}_{z=z_{0}} .
\end{aligned}
$$

On a prouvé la seconde égalité de la formule 5. (1) et (3) entraînent encore que pour $z \in \Delta_{0}$

$$
\int_{C\left(z_{0}\right)} \frac{\partial v(z, w)}{\partial z_{i}} \sigma^{*}\left(z_{0}, w\right)=\frac{\partial}{\partial z_{i}}\|\sigma(z, \cdot)\|_{D(z)}^{2} \cdot
$$

En appliquant la formule de Stokes au premier membre, on obtient la première égalité. La formule 5 est prouvée.

Puisque on ne utilise pas de calcul de $\S 2$, la première égalité est vraie pour le triple général $(\mathfrak{D}, \Delta, \pi)$ (ne pas nécessairement satisfaisant à la condition $(L))$. Comme le $1^{\text {er }}$ cas de $\S 6$, les deux formules ci-dessus entraîne que pour toute 1-forme $\sigma(z, \cdot)$ sur $\mathfrak{D}(z)$ de la forme (7.1), la réciproque de

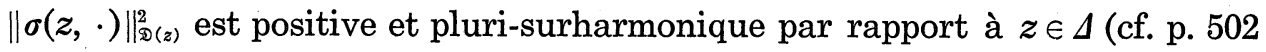
de [15]). 
Sous la configuration de la formule 3 , on a une formule de variation pour des périodes de la fonction harmonique conjuguée $H_{f}^{*}(z, w)$ de $H_{f}(z, w)$ pour $w \in \mathfrak{D}(z)$.

Formule 6. Soit $\gamma(z)$ une courbe fermée sur $\mathfrak{D}(z)$ qui se varie continûment avec $z \in \Delta$. Il vient alors pour

$$
\begin{aligned}
& \Omega_{\gamma}(z, \cdot)=\sigma_{\gamma(z)}(w)+\sqrt{-1} \sigma_{\gamma(z)}^{*}(w) \quad \text { sur } \mathfrak{D}(z), \\
& \frac{\partial}{\partial z_{i}} \int_{\gamma(z)} d H_{f}^{*}(z, w)=\left(\Omega_{\gamma}(z, \cdot), \frac{\partial^{2} H_{f}}{\partial \bar{z}_{i} \partial w} d w\right)_{D(z)} ; \\
& \frac{\partial^{2}}{\partial z_{i} \partial \bar{z}_{j}} \int_{\gamma(z)} d H_{f}^{*}(z, w) \\
& \quad=\left(\frac{\partial \Omega_{\gamma}(z, \cdot)}{\partial \bar{z}_{j}}, \frac{\partial^{2} H_{J}}{\partial \bar{z}_{i} \partial w}\right)_{D(z)}+\left(\frac{\partial^{2} H_{f}}{\partial \bar{z}_{j} \partial w} d w, \frac{\partial \Omega_{\gamma}(z, \cdot)}{\partial \bar{z}_{i}}\right)_{D(z)} .
\end{aligned}
$$

Preuve. Posons $u(z, w)=H_{f}(z, w)$ et $v(z, w)=\int_{\zeta_{0}(z)}^{w} \sigma_{r(z)}(z, w)$ sur $\mathfrak{D}(z)$. Dans la preuve de la formule 5 , on applique ces $u, v$ à l'expression (2.8) au lieu de (2.7). Alors le même raisonnement que la formule 5 nous conduit à celle 6 .

\section{nelles \\ §8. Quelques inégalités et une équation aux dérivées fonction-}

On observe des relations entre les formules dans $\S \mathrm{s}$ précédentes.

$1^{\circ}$ ) Soit $(\mathfrak{D}, \Delta, \pi)$ un triple satisfaisant aux conditions $(L),(T)$, et $\alpha$ une section analytique de $\mathfrak{D} \operatorname{sur} \Delta$. Soit $f$ une fonction sur $\partial \mathfrak{D} d u 2^{\text {ième }}$ cas à la fin de § 6. Alors, on a d'après la formule 3 ,

$$
\begin{aligned}
& \left|\frac{\partial^{2}}{\partial z_{i} \partial \bar{z}_{j}} H_{f}(z, \alpha(z))\right|^{2} \\
& \leqq \frac{4}{\pi^{2}}\left\{\iint_{\triangleright(z)}\left(\left|\frac{\partial^{2} H_{f}}{\partial \bar{z}_{i} \partial \bar{w}}\right|\left|\frac{\partial^{2} g_{\alpha}}{\partial \bar{z}_{j} \partial w}\right|+\left|\frac{\partial^{2} H_{f}}{\partial \bar{z}_{j} \partial w}\right|\left|\frac{\partial^{2} g_{\alpha}}{\partial z_{i} \partial \bar{w}}\right|\right) d u d v\right\}^{2} .
\end{aligned}
$$

Par l'inégalité de Schwarz, il vient

$$
\leqq \frac{4}{\pi^{2}} \iint_{D(z)}\left(\left|\frac{\partial^{2} H_{f}}{\partial z_{i} \partial \bar{w}}\right|^{2}+\left|\frac{\partial^{2} H_{f}}{\partial \bar{z}_{j} \partial w}\right|^{2}\right) d u d v \cdot \iint_{D(z)}\left(\left|\frac{\partial^{2} g_{\alpha}}{\partial \bar{z}_{j} \partial w}\right|^{2}+\left|\frac{\partial^{2} g_{\alpha}}{\partial z_{i} \partial \bar{w}}\right|^{2}\right) d u d v
$$

D'après la formule 2 et l'expression (6.7), il vient

$$
\leqq \frac{1}{\pi}\left(\frac{\partial^{2}}{\partial z_{i} \partial \bar{z}_{i}}+\frac{\partial^{2}}{\partial z_{j} \partial \bar{z}_{j}}\right) I(\bar{f}, z) \cdot\left(\frac{\partial^{2}}{\partial z_{i} \partial \bar{z}_{i}}+\frac{\partial^{2}}{\partial z_{j} \partial \bar{z}_{j}}\right)\left(-\lambda_{\alpha}(z)\right) .
$$


Pareillement, on a d'après les formules 5, 6,

$$
\begin{aligned}
& \left|\frac{\partial^{2}}{\partial z_{i} \partial \bar{z}_{j}} \int_{\tau(z)} d H_{f}^{*}(z, w)\right|^{2} \\
& \quad \leqq\left(\frac{\partial^{2}}{\partial z_{i} \partial \bar{z}_{i}}+\frac{\partial^{2}}{\partial z_{j} \partial \bar{z}_{j}}\right) I(\bar{f}, z) \cdot\left(\frac{\partial^{2}}{\partial z_{i} \partial \bar{z}_{i}}+\frac{\partial^{2}}{\partial z_{j} \partial \bar{z}_{j}}\right)\left\|\sigma_{r}(z, \cdot)\right\|_{\triangleright(z)}^{2} \cdot
\end{aligned}
$$

En posant $i=j$ dans les inégalités ci-dessus, on obtient un principe: la variation d'ordre deux de la forme $\delta \bar{\delta}$ par rapport à $z$ de la valeur, ou la période de la conjuguée, de la solution $H_{f}$ du problème de Dirichlet sur $\mathfrak{S}(z)$ est plus legère que celle de l'intégrale de Dirichlet $I(\bar{f}, z)$ étendue à $\mathfrak{(}(z)$.

$2^{\circ}$ ) Soit $(\mathfrak{D}, \Delta, \pi)$ un triple satisfaisant aux conditions $(L),(T)$, et $\alpha, \beta$ deux sections analytiques de $\mathfrak{D}$ sur 4 . Par l'inégalité de Schwarz, les formules 1 et 2 nous conduisent à l'inégalité (cf. p. 543 de [4]):

$$
\left|\frac{\partial^{2} g_{\alpha}(z, \beta(z))}{\partial z_{i} \partial \bar{z}_{j}}\right|^{2} \leqq \frac{1}{4}\left(\frac{\partial^{2}}{\partial z_{i} \partial \bar{z}_{i}}+\frac{\partial^{2}}{\partial z_{j} \partial \bar{z}_{j}}\right)\left(-\lambda_{\alpha}(z)\right) \cdot\left(\frac{\partial^{2}}{\partial z_{i} \partial \bar{z}_{i}}+\frac{\partial^{2}}{\partial z_{j} \partial \bar{z}_{j}}\right)\left(-\lambda_{\beta}(z)\right)
$$

$3^{\circ}$ ) Étant donnée une suite de sections analytiques $\left\{\alpha_{\nu}\right\}$ de $\mathfrak{D}$ tendant uniformément vers celle de $\alpha$ de façon que $\alpha_{\nu}(z) \neq \alpha(z)$ pour aucun $z \in \Delta$, la série $\left\{\left(\partial^{2} g_{\alpha_{\nu}} / \partial \bar{z}_{i} \partial w\right) d w\right\}_{\nu=1,2, \ldots}$ converge vers $\left(\partial^{2} g_{\alpha} / \partial \bar{z}_{i} \partial w\right) d w$ dans l'espace hilbertien $\Gamma_{h}(\mathfrak{S}(z))$. D'apres les formules 1,2 , on en a une égalité

$$
\lim _{\nu \rightarrow \infty} \frac{\partial^{2} g_{\alpha}\left(z, \alpha_{\nu}(z)\right)}{\partial z_{i} \partial \bar{z}_{j}}=\frac{\partial^{2} \lambda_{\alpha}(z)}{\partial z_{i} \partial \bar{z}_{j}}
$$

Ce n'est pas trivial, parce que la même égalité limite pour la différentielle de la forme $\partial z_{i}$ ou $\partial z_{i} \partial z_{\jmath}$ n'est pas vraie.

$\left.4^{\circ}\right)$ On va comparer la formule 1 avec celle de variation d'Hadamard bien nonnue (p. 519 de [4]). On rappelle briévement cette formule: soit $\Delta=$ $\{|z|<\rho\}$ un disque du plan $z$, et $D(z)$ où $z \in \Delta$ un ouvert connexe multivalent étalé sans points de ramification au-dessus du plan $C=\{|w|<\infty\}$ tel que la frontière $\partial D(z)=C(z)$ de $D(z)$ dans $C$ consiste en nombre fini de courbes fermées de Jordan analytique au sens réel et $C(z)$ se varie analyiquement au sens réel avec $z \in \Delta$. Pour $a, b \in D(z)$, on dénote par $g_{a}(z, b)$ la valeur au point $b$ de la fonction de Green sur $D(z)$ avec pôle $a$. Soit $z$ fixé dans $\Delta$ et soit $\zeta \in C(z)$. On écrit $\boldsymbol{n}_{\zeta}$ le vecteur normal extérieur unité de la courbe $C(z)$ au point $\zeta$. Si $\delta z$ est assez petit, $z+\delta z \in \Delta$ et la normale de $C(z)$ à $\zeta$ rentre $C(z+\delta z)$ une seule foi dans un voisinage de $\zeta$, dont l'intersection on pose $\zeta-\delta n(z)_{\zeta} \cdot n_{\zeta}\left(\delta n(z)_{\zeta}\right.$ étant réel). Il vient par notation (3.4)

$$
g_{a}(z+\delta z, b)-g_{a}(z, b)=-\frac{1}{2 \pi} \int_{C(z)} \frac{\partial g_{a}(z, \zeta)}{\partial n_{\zeta}} \frac{\partial g_{b}(z, \zeta)}{\partial n_{\zeta}} \delta n(z)_{\zeta} d s_{\zeta}+o(|\delta z|)
$$


tel que $\lim _{r \rightarrow 0} o(r)=0$. Cette égalité s'écrit simplement

$$
\delta g_{a}(b)=-\frac{1}{2 \pi} \int_{c} \frac{\partial g_{a}}{\partial n} \frac{\partial g_{b}}{\partial n} \delta n d s
$$

qui s'appelle la formule de variation d'Hadamard dans p. 182 de [8]. En autre côté, la nôtre est suivante: quand $C(z)$ se varie analytiquement (au sens complexe) avec $z \in \Delta$, c'est-à-dire, $z \rightarrow D(z)$ où $z \in \Delta$ est variation analytique au sens de $\S 2$, on a trouvé

$$
\frac{\partial^{2}}{\partial z \partial \bar{z}} g_{a}(z, b)=-\frac{4}{\pi} \operatorname{Re}\left\{\iint_{D(z)} \frac{\partial^{2} g_{a}(z, w)}{\partial \bar{z} \partial w} \frac{\partial^{2} g_{b}(z, w)}{\partial z \partial \bar{w}} d u d v\right\} .
$$

Plus simplement elle s'écrit

$$
\partial \bar{\partial} g_{a}(b)=-\frac{4}{\pi} \operatorname{Re}\left\{\iint_{D} \bar{\partial} \frac{\partial g_{a}}{\partial w} \partial \frac{\partial g_{b}}{\partial \bar{w}} d u d v\right\} .
$$

De la ressemblance de l'expression (8.2') avec celle (8.1), des résultats obtenus par (8.1) nous enseignent ceux correspondants par (8.2') comme suit.

(a) Le même augument que p. 1056 de [4] entraîne que, pour $m, n$ entiers positifs,

$$
\partial \bar{\partial} \frac{\partial^{m+n} g_{a}(b)}{\partial \bar{a}^{m} \partial b^{n}}=-\frac{2}{\pi} \iint_{D}\left(\bar{\partial} \frac{\partial^{m+1} g_{a}}{\partial \bar{a}^{m} \partial w} \partial \frac{\partial^{n+1} g_{b}}{\partial b^{n} \partial \bar{w}}+\partial \frac{\partial^{m+1} g_{a}}{\partial \bar{a}^{m} \partial \bar{w}} \bar{\partial} \frac{\partial^{n+1} g_{b}}{\partial b^{n} \partial w}\right) d u d v .
$$

En particulier, on a l'inégalité pour $m \geqq 1$

$$
\left\{\partial \bar{\partial} \frac{\partial^{2 m} g_{a}(b)}{\partial \bar{a}^{m} \partial b^{m}}\right\}_{a=b}=-\frac{2}{\pi} \iint_{D}\left(\left|\bar{\partial} \frac{\partial^{m+1} g_{a}}{\partial \bar{a}^{m} \partial w}\right|^{2}+\left|\partial \frac{\partial^{m+1} g_{a}}{\partial \bar{a}^{m} \partial \bar{w}}\right|^{2}\right) d u d v \leqq 0 .
$$

(b) En faisant $\partial^{2} / \partial \bar{a} \partial b$-différentielle de (8.1) et posant

$$
\Phi(a, b)=\partial^{2} g_{a}(b) / \partial \bar{a} \partial b
$$

il vient $\Phi(a, b)=\overline{\Phi(b, a)}$ et $\Phi(a, b)$ vérifie à l'équation aux dérivées fonctionnelles (p. 547 de [4]):

$$
\delta \Phi(a, b)=-\frac{1}{2 \pi} \int_{c} \Phi(a, w) \Phi(w, b) \delta n d s
$$

qui s'appelle l'équation d'Hadamard dans p. 167 de [8]. Elle est une base du développement de la recherche de P. Lévy [7]. Pour obtenir notre équation correspondante à celle (8.3), on fait des remarques:

(1) La fonction $\Phi(a, w)$ ci-dessus est définie non seulement pour $a, w \in D$, mais aussi pour $a, w \in \partial D$ (cf. la fonction $\mathscr{G}_{a}^{w}$ en p. 176 de P. Lévy [8]). Le cas 
où $a \in D, \Phi(a, w)$ est holomorphe pour $w \in D \cup \partial D$, et le cas où $a \in \partial D$, elle l'est aussi pour $w \in D \cup \partial D-\{a\}$, et se développe dans un voisinage de $a$

$$
\Phi(a, w)=\frac{-\exp \left(2 \sqrt{-1} \Theta^{*}(a, \partial D)\right)}{(w-a)^{2}}+\sum_{n=0}^{\infty} A_{n}(w-a)^{n}
$$

où on note, pour $\zeta \in \partial D, \Theta(\zeta, \partial D)$ (resp. $\Theta^{*}(\zeta, \partial D)$ ) l'angle du vecteur tangentiel (resp. normal extérieur) de la courbe $\partial D$ au point $\zeta: \Theta^{*}(\zeta, \partial D)=\Theta(\zeta, \partial D)-\pi / 2$.

(2) Il est bien connu (p. 532 de M. Schiffer [11] et p. 59 de S. Bergmann [3]) que $(-2 / \pi) \Phi(a, w)$ pour $a, w \in D$ n'est autre que la fonction de Bergmann $K(a, w)$ telle que

$$
\iint_{D} \overline{K(a, w)} f(w) d u d v=f(a)
$$

pour toute $f(w)$ holomorphe sur $D \cup \partial D$ et

$$
\iint_{D} \overline{K(b, w)} \dot{K}(a, w) d u d v=K(a, b)
$$

pour $a, b \in D$. Mais, si $a, b$ sont sur $\partial D$, d'après (8.4) l'intégrale n'existe pas en général. On aura besoin du suivant: pour $a, b \in \partial D$ et $a \neq b$, il vient

$$
\begin{aligned}
& \Phi(a, b)=\lim _{\varepsilon \rightarrow 0} \Phi_{s}(a, b) ; \\
& \Phi_{s}(a, b)=-\frac{1}{\pi} \iint_{D_{s}} \Phi(a, w) \Phi(w, b) d u d v ; \\
& D_{\varepsilon} \subset D \text { pour } \varepsilon>0 \text { et } D_{\varepsilon} \longrightarrow D \text { pour } \varepsilon \longrightarrow 0 ; \\
& D_{\varepsilon}=\{w \in D: d(w, \partial D)>\varepsilon\},
\end{aligned}
$$

$d(w, \partial D)$ étant la distance euclidienne sur $D$ entre $w$ et $\partial D$.

En effet, on écrit $\partial D_{\varepsilon}=C_{\varepsilon}$ et $\partial D=C$. Pour $\zeta \in C$, on pose $\zeta_{s}$ l'intersection de $C_{\varepsilon}$ avec la normale de $C$ à $\zeta$. Il vient $\Theta(\zeta, C)=\Theta\left(\zeta_{s}, C_{s}\right)$. Quand on considère la transformation $\tilde{w}=\varphi(w)=(w-b) \exp (-\sqrt{-1} \Theta(b, C))$, on peut supposer, par $\Phi(a, b) \overline{d a} d b=\tilde{\Phi}(\varphi(a), \varphi(b)) d \overline{\varphi(a)} d \varphi(b)$, que $b$ est l'origine $O$ du plan $w$ et $\Theta(b, C)=0$, d'où $b_{\varepsilon}=\sqrt{-1} \beta_{s}\left(\beta_{\varepsilon}:\right.$ réel positif $)$. Soit donné $\rho>0$ petit et soit $\delta^{\rho}(a)$ (resp. $\left.\delta^{\rho}(b)\right)$ le disque de centre $a$ (resp. $b$ ) et rayon $\rho$ tel que $\delta^{\rho}(a) \cap$ $\delta^{\rho}(b)=\phi$. On pose $\gamma_{\varepsilon}^{\rho}(a)$ (resp. $\gamma_{s}^{\rho}(b)$; resp. $\gamma^{\rho}(a)$; resp. $\left.\gamma^{\rho}(b)\right)=C_{\varepsilon} \cap \delta^{\rho}(a)$ (resp. $C_{\varepsilon} \cap \delta^{\rho}(b)$; resp. $C \cap \delta^{\rho}(a)$; resp. $\left.C \cap \delta^{\rho}(b)\right)$. Pour $w \in \gamma_{\varepsilon}^{\rho}(b)$, il vient par $\Theta\left(b_{\varepsilon}, C_{\varepsilon}\right)$ $=0$,

$$
w-b=w=\overline{w-b}+2 \sqrt{-1} \beta_{\mathrm{s}}+O\left(|w-b|^{2}\right),
$$

où $O\left(|w-b|^{2}\right) /|w-b|^{2}$ est uniformément borné pour $w \in \gamma_{\varepsilon}^{\rho}(b)$ et $0<\varepsilon \ll 1$. D'après $g_{w_{1}}(w)=0$ pour $w_{1} \in \delta^{\rho}(a)$ et $w \in \gamma^{\rho}(b)$, il vient $\left(\partial g_{a} / \partial \bar{a}\right)(w)=0$ pour $w \in$ 
$\gamma^{\rho}(b)$, d'où $\left(\partial^{2} g_{a} / \partial \bar{a} \partial w\right) d w+\left(\partial^{2} g_{a} / \partial \bar{a} \partial \bar{w}\right) d \bar{w}=0$ pour $w$ le long de $\gamma^{\rho}(b)$. Il s'ensuit $\operatorname{par} \Theta(b, C)=0$ que

$$
\frac{\partial g_{a}}{\partial \bar{a}}(b)=0 ; \quad \frac{\partial^{2} g_{a}}{\partial \bar{a} \partial w}(b)+\frac{\partial^{2} g_{a}}{\partial \bar{a} \partial \bar{w}}(b)=0 .
$$

En posant $C_{\varepsilon}^{\rho}=C_{s}-\gamma_{\varepsilon}^{\rho}(a)-\gamma_{\varepsilon}^{\rho}(b)$, on a grâce à la formule de Stokes

$$
\begin{aligned}
\Phi_{\varepsilon}(a, b) & =\frac{1}{2 \pi \sqrt{-1}}\left(\int_{C_{\varepsilon}^{\rho}}+\int_{\tau_{\varepsilon}^{\rho}(a)}+\int_{\tau_{\varepsilon}^{\rho}(b)}\right) \frac{\partial g_{a}}{\partial \bar{a}} \frac{\partial^{2} g_{b}}{\partial b \partial \bar{w}} d \bar{w} \\
& =I_{1}(\rho, \varepsilon)+I_{2}(\rho, \varepsilon)+I_{3}(\rho, \varepsilon) .
\end{aligned}
$$

Puisque $\partial g_{a} / \partial \bar{a}$ est régulière et nulle sur $C-\gamma^{\rho}(a)-\gamma^{\rho}(b), I_{1}(\rho, \varepsilon)$ tend vers zéro pour $\varepsilon \rightarrow 0$. On a pour $w \in \delta^{\rho}(a)$

$$
\frac{\partial g_{a}}{\partial \bar{a}}(w)=\frac{1}{\overline{w-a}}+\frac{\exp \left(2 \sqrt{-1} \Theta^{*}(a, C)\right)}{w-a}+O(1) .
$$

Il vient par $\Theta\left(a_{s}^{\prime}, C_{s}\right)=\Theta(a, C)$ que pour $w$ le long de $\gamma_{s}^{\rho}(a)$

$$
\frac{d \bar{w}}{d w}=\exp \left(-2 \sqrt{-1} \Theta\left(w, C_{\varepsilon}\right)\right)=\exp (-2 \sqrt{-1} \Theta(a, C))+O(|w-a|) .
$$

Il s'ensuit que pour $w$ le long de $\gamma_{s}^{\rho}(a)$

$$
\begin{aligned}
& \frac{\partial^{2} g_{b}}{\partial b \partial \bar{w}} \frac{\partial g_{a}}{\partial \bar{a}} d \bar{w} \\
& \quad=\left(\frac{\partial^{2} g_{b}}{\partial b \partial \bar{w}}(a)+O(|w-a|)\right) \cdot\left(\frac{1}{\overline{w-a}}+\frac{\exp \left(2 \sqrt{-1} \Theta^{*}(a, C)\right)}{w-a}+O(1)\right) d \bar{w} \\
& \quad=-2 \sqrt{-1} \frac{\partial^{2} g_{a}(b)}{\partial \bar{a} \partial b} \operatorname{Im}\{d \log (w-a)\}+O(1)|d w| .
\end{aligned}
$$

On en a $\lim _{\varepsilon \rightarrow 0} I_{2}(\rho, \varepsilon)=\partial^{2} g_{a}(b) / \partial \bar{a} \partial b+J_{2}(\rho)$ tel que $J_{2}(\rho) \rightarrow 0$ pour $\rho \rightarrow 0$. D'après (8.6) et (8.7), on a

$$
\begin{aligned}
\frac{\partial g_{a}}{\partial \bar{a}}(w) & =\frac{\partial g_{a}}{\partial \bar{a}}(b)+\frac{\partial^{2} g_{a}}{\partial w \partial \bar{a}}(b)(w-b)+\frac{\partial^{2} g_{a}}{\partial \bar{w} \partial \bar{a}}(b) \overline{w-b}+O\left(|w-b|^{2}\right) \\
& =\left(2 \sqrt{-1} \frac{\partial^{2} g_{a}(b)}{\partial \bar{a} \partial b}\right) \cdot \beta_{s}+O\left(|w-b|^{2}\right) .
\end{aligned}
$$

Il vient d'après $(8.4)$ et $\Theta^{*}(b, C)=-\pi / 2$ que pour $w$ le long de $\gamma_{e}^{o}(b)$

$$
\begin{aligned}
\frac{\partial^{2} g_{a}}{\partial \bar{a}} & \frac{\partial^{2} g_{b}}{\partial b \partial \bar{w}} d \bar{w} \\
\quad & =\left(\left(2 \sqrt{-1} \frac{\partial^{2} g_{a}(b)}{\partial \bar{a} \partial b}\right) \cdot \beta_{s}+O\left(|w-b|^{2}\right)\right) \cdot\left(\frac{1}{\overline{w-b}^{2}}+\sum_{n=0}^{\infty} \bar{B}_{n}(\overline{w-b})^{n}\right) d \bar{w}
\end{aligned}
$$




$$
=\left(-2 \sqrt{-1} \frac{\partial^{2} g_{a}(b)}{\partial \bar{a} \partial b}\right) \cdot \beta_{\varepsilon} d\left(\frac{1}{\overline{w-b}}\right)+O(1)|d w|
$$

d'où $I_{3}(\rho, \varepsilon)=(-1 / \pi)\left(\partial^{2} g_{a}(b) / \partial \bar{a} \partial b\right) \cdot \beta_{\varepsilon} \cdot\left[2 \rho /\left(\rho^{2}+\varepsilon^{2}\right)\right]+J_{3}(\rho, \varepsilon)$ tel que, si $\rho \rightarrow 0, J_{3}(\rho, \varepsilon)$ $\rightarrow 0$ uniformément pour $0<\varepsilon \ll 1$. Or, soit donné $\eta>0$ à periori. D'abord on trouve et fixe un $\rho_{0}>0$ de façon que $J_{2}\left(\rho_{0}\right)<\eta / 5$ et $J_{3}\left(\rho_{0}, \varepsilon\right)<\eta / 5$ pour tout $0<$ $\varepsilon \ll 1$, et ensuite choisit $\varepsilon_{0}>0$ dépendant de $\rho_{0}$ tel que, pour tout $0<\varepsilon<\varepsilon_{0}$, il vienne $I_{1}\left(\rho_{0}, \varepsilon\right)<\eta / 5,\left|I_{2}\left(\rho_{0}, \varepsilon\right)-\left(\partial^{2} g_{a}(b) / \partial \bar{a} \partial b+J_{2}\left(\rho_{0}\right)\right)\right|<\eta / 5$ et, d'après $\beta_{s} \rightarrow 0$ pour $\varepsilon \rightarrow 0,\left|I_{3}\left(\rho_{0}, \varepsilon\right)-J_{3}\left(\rho_{0}, \varepsilon\right)\right|<\eta / 5$. On en a $\left|\Phi_{\varepsilon}(a, b)-\partial^{2} g_{a}(b) / \partial \bar{a} \partial b\right|<\eta$ pour $0<\varepsilon<$ $\varepsilon_{0}$, d'où (8.5).

Maintenant, on rentre à l'expression (3.6): pour $a, w \in D$ et $z \in \Delta_{0}$ il vient

$$
g_{a}(z, w)-g_{a}\left(z_{0}, w\right)=\frac{\sqrt{-1}}{\pi} \int_{C\left(z_{0}\right)} g_{w}\left(z, w_{1}\right) \frac{\partial g_{a}\left(z_{0}, w_{1}\right)}{\partial w_{1}} d w_{1} .
$$

Par $\partial^{3} / \partial z \partial \bar{a} \partial \bar{w}$-différentielle, il vient

$$
\frac{\partial^{3} g_{a}(z, w)}{\partial z \partial \bar{a} \partial \bar{w}}=\frac{\sqrt{-1}}{\pi} \int_{C\left(z_{0}\right)} \frac{\partial^{2} g_{w}\left(z, w_{1}\right)}{\partial z \partial \bar{w}} \frac{\partial^{2} g_{a}\left(z_{0}, w_{1}\right)}{\partial \bar{a} \partial w_{1}} d w_{1}
$$

D’après $\left(4.3^{\prime}\right)$, il vient en abrégé $\xi^{\prime}=\partial \xi / \partial z$,

$$
\frac{\partial^{3} g_{a}}{\partial z \partial \bar{a} \partial \bar{w}}\left(z_{0}, w\right)=\frac{-\sqrt{-1}}{\pi} \int_{C\left(z_{0}\right)} \frac{\partial^{2} g_{w}\left(z_{0}, w_{1}\right)}{\partial w_{1} \partial \bar{w}} \frac{\partial^{2} g_{a}\left(z_{0}, w_{1}\right)}{\partial \bar{a} \partial w_{1}} \xi^{\prime}\left(z_{0}, w_{1}\right) d w_{1} .
$$

On en a pour $a, w \in D$ et $z \in \Delta$

$$
\frac{\partial^{3} g_{a}(z, w)}{\partial z \partial \bar{a} \partial \bar{w}}=\frac{-\sqrt{-1}}{\pi} \int_{C(z)} \Phi\left(z, w, w_{1}\right) \Phi\left(z, a, w_{1}\right) \xi^{\prime}\left(z, w_{1}\right) d w_{1} .
$$

Pareillement il vient pour $a, b, w \in D$ et $z \in \Delta$

$$
\begin{aligned}
& \frac{\partial^{3} g_{b}(z, w)}{\partial \bar{z} \partial b \partial w}=\frac{\sqrt{-1}}{\pi} \int_{C(z)} \Phi\left(z, w_{2}, w\right) \Phi\left(z, w_{2}, b\right) \overline{\xi^{\prime}\left(z, w_{2}\right)} d \bar{w}_{2} \\
& \frac{\partial^{3} g_{a}(z, w)}{\partial \bar{z} \partial \bar{a} \partial w}=\frac{-\sqrt{-1}}{\pi} \int_{C(z)} \Phi\left(z, w_{1}, w\right) \Phi\left(z, a, w_{1}\right) \overline{\xi^{\prime}\left(z, w_{1}\right)} d w_{1} \\
& \frac{\partial^{3} g_{b}(z, w)}{\partial z \partial b \partial \bar{w}}=\frac{\sqrt{-1}}{\pi} \int_{C(z)} \Phi\left(z, w, w_{2}\right) \Phi\left(z, w_{2}, b\right) \xi^{\prime}\left(z, w_{2}\right) d \bar{w}_{2} .
\end{aligned}
$$

Par $\partial^{2} / \partial \bar{a} \partial b$-différentielle de l'expression (8.2), on a pour $a, b \in D$ et $z \in \Delta$

$$
\frac{\partial^{4} g_{a}(z, b)}{\partial z \partial \bar{z} \partial \bar{a} \partial b}=\frac{-2}{\pi} \iint_{D(z)}\left(\frac{\partial^{3} g_{a}(z, w)}{\partial \bar{z} \partial \bar{a} \partial w} \frac{\partial^{3} g_{b}(z, w)}{\partial z \partial b \partial \bar{w}}+\frac{\partial^{3} g_{a}(z, w)}{\partial z \partial \bar{a} \partial \bar{w}} \frac{\partial^{3} g_{b}(z, w)}{\partial \bar{z} \partial b \partial w}\right) d u d v
$$

En substituant quatre expression ci-dessus à cette égalité, on obtient 


$$
\begin{array}{r}
\frac{\partial^{2} \Phi(z, a, b)}{\partial z \partial \bar{z}}=\frac{-4}{\pi^{3}} \iint_{D(z)}\left[\int_{C(z)} \int_{C(z)}\left(\operatorname{Re}\left\{\Phi\left(z, w_{1}, w\right) \Phi\left(z, w, w_{2}\right) \overline{\xi^{\prime}\left(z, w_{1}\right)} \xi^{\prime}\left(z, w_{2}\right)\right\}\right)\right. \\
\left.. \Phi\left(z, a, w_{1}\right) \Phi\left(z, w_{2}, b\right) d w_{1} d \bar{w}_{2}\right] d u d v
\end{array}
$$

D’après le théorème de Fubini, il vient

$$
\begin{aligned}
&=\lim _{\varepsilon \rightarrow 0} \frac{4}{\pi^{2}} \int_{C(z)} \int_{C(z)}( \operatorname{Re}\left\{\frac{-1}{\pi} \iint_{D_{\varepsilon}(z)} \Phi\left(z, w_{1}, w\right) \Phi\left(z, w, w_{2}\right) d u d v\right. \\
&\left.\left.\cdot \overline{\xi^{\prime}\left(z, w_{1}\right)} \xi^{\prime}\left(z, w_{2}\right)\right\}\right) \Phi\left(z, a, w_{1}\right) \Phi\left(z, w_{2}, b\right) d w_{1} d \bar{w}_{2}
\end{aligned}
$$

où $D_{s}(z) \in D(z)$ pour $\varepsilon>0$ et $D_{s}(z) \rightarrow D(z)$ comme $\varepsilon \rightarrow 0$. Enfin par notation (8.5) on arrive à une équation suivante aux dérivées fonctionnelles pour la variation analytique quelconque:

$$
\begin{aligned}
\frac{\partial^{2} \Phi(z, a, b)}{\partial z \partial \bar{z}}=\lim _{\varepsilon \rightarrow 0} \frac{4}{\pi^{2}} \int_{C(z)} \int_{C(z)}\left(\operatorname{Re}\left\{\Phi_{\varepsilon}\left(z, w_{1}, w_{2}\right) \overline{\xi^{\prime}\left(z, w_{1}\right)} \xi^{\prime}\left(z, w_{2}\right)\right\}\right) \\
\times \Phi\left(z, a, w_{1}\right) \Phi\left(z, w_{2}, b\right) d w_{1} d \bar{w}_{2}
\end{aligned}
$$

telle que $\lim _{s \rightarrow 0} \Phi_{\varepsilon}\left(z, w_{1}, w_{2}\right)=\Phi\left(z, w_{1}, w_{2}\right)$ pour $w_{1}, w_{2} \in C(z)$. Pour la commodité, on l'écrit

$$
\partial \bar{\partial} \Phi(a, b)=\frac{4}{\pi^{2}} \int_{C} \int_{C}\left(\operatorname{Re}\left\{\Phi\left(w_{1}, w_{2}\right) \partial \bar{w}_{1} \partial w_{2}\right\}\right) \Phi\left(a, w_{1}\right) \Phi\left(w_{2}, b\right) d w_{1} d \bar{w}_{2} .
$$

(c) On sait (p. 548 d'Hadamard [4]) beaucoup d'exemples de $\Phi$ qui verifient à l'équation (8.3) et il existe certainement autres exemples de $\Phi$ que $\partial^{2} g_{a}(b) /$ $\partial \bar{a} \partial b$ qui verifient à (8.8). Concernant ces exemples et des études de (8.8) analogues à ceux de (8.3) faites dans p. 549 de [4] et P. Lévy [7], ils seront traités ultérieurement. On examine ici le second membre de (8.8) pour $\Phi(a, b)$ $=\partial^{2} g_{a}(b) / \partial \bar{a} \partial b$ au point $z=0$ en cas le plus simple: $D(0)=\{|w|<1\}$. On a $D_{\varepsilon}(0)$ $=\{|w|<1-\varepsilon\}$ et $\xi^{\prime}(0, \exp (\sqrt{-1} \theta))=f(\theta)$ étant fonction définie sur $C=\partial D(0)=$ $\{\exp (\sqrt{-1} \theta): 0 \leqq \theta \leqq 2 \pi\}$. On a, par calcul direct,

$$
\begin{aligned}
& \Phi(a, b)=\frac{-1}{(1-\bar{a} b)^{2}} \quad \text { pour } a, b \in D(0) \cup C ; \\
& \Phi_{s}\left(w_{1}, w_{2}\right)=\frac{-(1-\varepsilon)^{2}}{\left(1-(1-\varepsilon)^{2} \bar{w}_{1} w_{2}\right)^{2}} \quad \text { pour } w_{1}, w_{2} \in C .
\end{aligned}
$$

Il vient comme (8.5) $\Phi_{\varepsilon}\left(w_{1}, w_{2}\right) \rightarrow \Phi\left(w_{1}, w_{2}\right)$ pour $\varepsilon \rightarrow 0$. Pour simplicité, on pose $r=1-\varepsilon(<1)$ et $w_{i}=\exp \left(\sqrt{-1} \theta_{i}\right)$ pour $w_{i} \in C$ et $i=1,2$. Alors on a $\Phi_{s}\left(w_{1}, w_{2}\right)$ $=-r^{2} \sum_{n=0}^{\infty}(n+1) r^{2 n} \exp \left(-\sqrt{-1} n \theta_{1}\right) \exp \left(\sqrt{-1} n \theta_{2}\right) ; \Phi\left(a, w_{1}\right)=-\sum_{m=0}^{\infty}(m+1)$ - $\bar{a}^{m} \exp \left(\sqrt{-1} m \theta_{1}\right) ; \Phi\left(w_{2}, b\right)=-\sum_{l=0}^{\infty}(l+1) b^{l} \exp \left(-\sqrt{-1} l \theta_{2}\right)$. Le second membre de (8.8) devient 


$$
\begin{aligned}
= & \lim _{r \rightarrow 1} \frac{-4 r^{2}}{\pi^{2}} \sum_{n, m, l=0}^{\infty}\left[(n+1)(m+1)(l+1) r^{2 n} \bar{a}^{m} b^{l}\right] \\
& \times\left[\int_{0}^{2 \pi} \overline{f\left(\theta_{1}\right)} \exp \left(\sqrt{-1}(m+1-n) \theta_{1}\right) d \theta_{1} \cdot \int_{0}^{2 \pi} f\left(\theta_{2}\right) \exp \left(-\sqrt{-1}(l+1-n) \theta_{2}\right) d \theta_{2}\right. \\
& \left.+\int_{0}^{2 \pi} f\left(\theta_{1}\right) \exp \left(\sqrt{-1}(m+1+n) \theta_{1}\right) d \theta_{1} \cdot \int_{0}^{2 \pi} \overline{f\left(\theta_{2}\right)} \exp \left(-\sqrt{-1}(l+1+n) \theta_{2}\right) d \theta_{2}\right] .
\end{aligned}
$$

Il s'ensuit que (8.8') s'exprime

$$
\begin{aligned}
\partial \bar{\partial} \Phi(a, b)=\lim _{r \rightarrow 1} \frac{-4}{\pi^{2}} \sum_{n, m, l=0}^{\infty}(n+1)(m+1)(l+1) r^{2 n} \bar{a}^{m} b^{l}\left(\overline{c_{-(m+1-n)}} \cdot c_{-(l+1-n)}\right. \\
\left.\quad+c_{m+1+n} \cdot \overline{c_{l+1+n}}\right) ; \\
c_{n}=\frac{1}{2 \pi} \int_{0}^{2 \pi} f(\theta) \exp (\sqrt{-1} n \theta) d \theta \quad(n=0, \pm 1, \pm 2, \cdots) .
\end{aligned}
$$

Il vient l'inégalité pour $a=b$ énoncée en $(a)$ pour $m=1$,

$$
\begin{aligned}
\partial \bar{\partial} \Phi(a, a) & =\frac{-4}{\pi^{2}} \sum_{n=0}^{\infty}(n+1)\left\{\left|\sum_{m=0}^{\infty}(m+1) c_{-(m+1-n)} a^{m}\right|^{2}+\left|\sum_{m=0}^{\infty}(m+1) \overline{c_{m+1+n}} a^{m}\right|^{2}\right\} \\
& \leqq 0
\end{aligned}
$$

En particulier pour $a=b=0$, il vient

$$
\partial \bar{\partial} \Phi(0,0)=\frac{-4}{\pi^{2}} \sum_{n=0}^{\infty}(n+1)\left(\left|c_{n-1}\right|^{2}+\left|c_{n+1}\right|^{2}\right) .
$$

On en remarque que le cas où $D(0)=\{|w|<1\}, \partial \bar{\partial} \Phi(0,0)$ soit nulle pour $z=0$ si et seulement si $\xi(z, w)$ est de la forme pour $\{|w|=1\}$ et $z \in \Delta$

$$
\xi(z, w)=w+\left[\sum_{n=2}^{\infty} a_{n} w^{n}\right] z+\left[\sum_{n=0}^{\infty}\left(b_{n} w^{n}+c_{n} \bar{w}^{n}\right)\right] z^{2}+\cdots ;
$$

$a_{n}, b_{n}, c_{n}$ étant constantes complexes.

$\left(c^{\prime}\right)$ Puisque $\Phi(a, b) d \bar{a} d b$ est invariante pour la transformation conforme $w \rightarrow \varphi(w)$, l'argument de $(c)$ donne autre expression de (8.8): pour $D_{s}$ de (8.5) tel que $D_{\varepsilon} \subset D$ et $\lim _{\varepsilon \rightarrow 0} D_{\varepsilon}=D$, on écrit $\partial D_{\varepsilon}=C_{\varepsilon}, \partial D=C$, et $w_{\varepsilon}\left(\in C_{\varepsilon}\right)$ pour $w \in$ $C$ l'intersection de $C_{\varepsilon}$ avec la normale de $C$ au point $w$. On considère la fonctionnelle $L_{\phi}$ sur un ensemble $E \times E$ de paire $(f, g)$ de fonctions définies sur $C$ de façon que

$$
\boldsymbol{L}_{\Phi}[f, g]=\lim _{\epsilon \rightarrow 0} \frac{1}{4 \pi^{2}} \int_{C} \int_{C} \Phi\left(w_{1 \varepsilon}, w_{2 \varepsilon}\right) f\left(w_{1}\right) \overline{g\left(w_{2}\right)}\left|d w_{1}\right|\left|d w_{2}\right|
$$

On peut prendre un $E$ dense dans l'espace $C^{\infty}(C)$ et trouve que $\overline{L_{\Phi}[f, g]}=$ $L_{\phi}[g, f] ; L_{\phi}[f, f] \leqq 0$ pour $f \in E$ et $L_{\phi}[f, f]=0$ si et seulement si $f=0$. Par cette notation, $\left(8.8^{\prime}\right)$ s'écrit symétriquement 
$\partial \bar{\partial} \Phi(a, b)=16\left\{L_{\Phi}[\Phi(a, w) \partial \bar{w} \exp (\sqrt{-1} \Theta(w)), \Phi(b, w) \partial \bar{w} \exp (\sqrt{-1} \Theta(w))]\right.$

$$
\left.+L_{\varphi}[\Phi(w, b) \partial \bar{w} \exp (-\sqrt{-1} \Theta(w)), \Phi(w, a) \partial \bar{w} \exp (-\sqrt{-1} \Theta(w))]\right\},
$$

où $\Theta(w)$ pour $w \in C$ désigne l'angle du vecteur tangentiel de $C$ au point $w$.

\section{§9. Equivalences entre des variations analytiques}

Etant données deux triples équivalents $\left(\mathfrak{D}_{i}, \Delta, \pi_{i}\right)(i=1,2)$, chaques deux fibres $\mathfrak{D}_{i}(z)\left(=\pi_{i}^{-1}(z)\right)$ où $z \in \Delta$ sont équivalentes comme surface de Riemann. On traite dans cette $\S$ le problème réciproque sur lequel on a obtenu quelques résultats affirmatifs (voir p. 523 de [15], p. 1121 de [16] et p. 619 de [17]).

Soient $R$ et $\tilde{R}$ deux surfaces de Riemann telles qu'il existe un difféomorphisme $\varphi$ de classe $C^{2}$ de $R$ sur $\tilde{R}$. Etant donnée 1 -forme $\tilde{\Omega}$ sur $\tilde{R}$, on écrit $\varphi^{\sharp} \widetilde{\Omega}$ celle sur $R$ tenue de $\widetilde{\Omega} \operatorname{par} \varphi$. On aura besoin $d u$

LEMme 4. Soient $(\mathfrak{D}, \Delta, \pi)$ et $(\tilde{D}, \Delta, \tilde{\pi})$ deux triples tels qu'il existe un difféomorphisme $\varphi$ de classe $C^{2}$ de $\mathfrak{D}$ sur $\mathfrak{D}$ tel que $\tilde{\pi} \circ \varphi=\pi$. Supposons que la restriction $\varphi(z, w)$ de $\varphi$ sur chaque fibre $\mathscr{D}(z)$ où $z \in \Delta$ soit analytique pour $w \in$ $\mathfrak{D}(z)$. Soit donnée sur chaque $\mathscr{D}(z)$ (resp. $\mathfrak{D}(z)$ ) une 1-forme holomorphe $\Omega(z, \cdot)$ (resp. $\tilde{\Omega}(z, \cdot))$ telle que

$$
\Omega(z, \cdot)=\varphi(z, \cdot)^{\#} \tilde{\Omega}(z, \cdot) .
$$

Alors on a pour $w \in \mathfrak{D}(z)$ et $i=1, \cdots, n$,

$$
\begin{aligned}
& \frac{\partial \Omega(z, \cdot)}{\partial \bar{z}_{i}}=\varphi(z, \cdot)^{\#} \frac{\partial \tilde{\Omega}(z, \cdot)}{\partial \bar{z}_{i}}+\frac{\partial F(z, w)}{\partial w} d w ; \\
& F(z, w)=\frac{\partial \varphi(z, w)}{\partial \bar{z}_{i}} \cdot \Omega(z, w) / \frac{\partial \varphi(z, w)}{\partial w} d w,
\end{aligned}
$$

où $F(z, w)$ est fonction uniforme dans $\mathfrak{D}$ dont la restriction sur chaque $\mathfrak{S}(z)$ où $z \in \Delta$ est holomorphe pour $w \in \mathfrak{D}(z)$.

Preuve. Pour que $F(z, w)$ soit fonction uniforme et holomorphe dans $\mathfrak{D}(z)$ il suffit de prouver que l'expression $\left(9.2^{\prime}\right)$ de $F(z, w)$ ne dépend pas de choix ni $\pi$-coordonnées de $\mathfrak{D}:(z, w) \in \Delta_{0} \times \delta$ où $\Delta_{0} \subset \Delta$ et $\delta=\{|w|<\tau\}$ ni celles de $\tilde{D}:(z, \tilde{w}) \in \Delta_{0} \times \tilde{\delta}$ où $\tilde{\delta}=\{|w|<\tilde{\tau}\}$. Car pour les autres $\left(z, w_{1}\right) \in \Delta_{0} \times \delta_{1}$ (resp. $\left.\left(z, \tilde{w}_{1}\right) \in \Delta_{0} \times \tilde{\delta}_{1}\right)$ de $\mathfrak{D}$ (resp. $\left.\mathfrak{D}\right)$, il existe une transformation analytique: $z=z$, $w_{1}=w_{1}(z, w)$ (resp. $\left.z=z, \tilde{w}_{1}=\tilde{w}_{1}(z, \tilde{w})\right)$ de $\Delta_{0} \times \delta$ (resp. $\left.\Delta_{0} \times \tilde{\delta}\right)$ sur $\Delta_{0} \times \delta_{1}$ (resp. $\Delta_{0} \times \tilde{\delta}_{1}$ ). On dénote par $\varphi$ (resp. $\varphi_{1}$ ) la transformation de $\Delta_{0} \times \delta$ (resp. $\Delta_{0} \times \delta_{1}$ ) sur $\Delta_{0} \times \tilde{\delta}$ (resp. $\Delta_{0} \times \tilde{\delta}_{1}$ ) conduite par $\varphi$ et pose $\Omega(z, \cdot)=h(z, w) d w$ (resp. $\left.h_{1}\left(z, w_{1}\right) d w_{1}\right)$ dans $\Delta_{0} \times \delta$ (resp. $\left.\Delta_{0} \times \delta_{1}\right)$. D'après $h_{1}\left(z, w_{1}\right)=h(z, w)\left(d w_{1} / d w\right)$ et $\varphi_{1}\left(z, w_{1}(z, w)\right)=\tilde{w}_{1}(z, \varphi(z, w))$ pour $(z, w) \in \Delta_{0} \times \delta$, on a évidemment 
$\left[\left(\partial \varphi / \partial \bar{z}_{i}\right) h /(\partial \varphi / \partial w)\right](z, w)=\left[\left(\partial \varphi_{1} / \partial \bar{z}_{i}\right) h_{1} /\left(\partial \varphi_{1} / \partial w_{1}\right)\right]\left(z, w_{1}\right)$ pour $w_{1}=w_{1}(z, w)$, ce qu'on demande.

Il s'agit de prouver (9.2) pour $\pi$-coordonnées ci-dessus $\Delta_{0} \times \delta$ (resp. $\left.\Delta_{0} \times \tilde{\delta}\right)$ de $\mathfrak{S}(\operatorname{resp} . \mathfrak{D})$. On pose $\Omega(z, w)(\operatorname{resp} . \tilde{\Omega}(z, \tilde{w}))=h(z, w) d w(\operatorname{resp} . \tilde{h}(z, \tilde{w}) d \tilde{w})$ dans $\Delta_{0} \times \delta$ (resp. $\left.\Delta_{0} \times \tilde{\delta}\right)$. D'après (9.1), on a $h(z, w)=\tilde{h}(z, \varphi(z, w))(\partial \varphi / \partial w)(z, w)$ pour $(z, w) \in \Delta_{0} \times \delta$. Par $w$-différentielle, on obtient

$$
\frac{\partial \tilde{h}}{\partial \tilde{w}}(z, \varphi(z, w))=\left\{\left(\frac{\partial h}{\partial w} \frac{\partial \varphi}{\partial w}-h \frac{\partial^{2} \varphi}{\partial w^{2}}\right) /\left(\frac{\partial \varphi}{\partial w}\right)^{3}\right\}_{(z, w)} .
$$

Par $\bar{z}_{i}$-différentielle on a aussi

$$
\begin{aligned}
\frac{\partial h}{\partial \bar{z}_{i}}(z, w)= & \left(\frac{\partial \tilde{h}}{\partial \bar{z}_{i}}(z, \varphi(z, w))+\frac{\partial \tilde{h}}{\partial \tilde{w}}(z, \varphi(z, w)) \frac{\partial \varphi}{\partial \bar{z}_{i}}(z, w)\right) \frac{\partial \varphi}{\partial w}(z, w) \\
& +\tilde{h}(z, \varphi(z, w)) \frac{\partial^{2} \varphi}{\partial \bar{z}_{i} \partial w}(z, w) .
\end{aligned}
$$

D'après deux égalités, on a pour $\tilde{w}=\varphi(z, w)$ et $(z, w) \in \Delta_{0} \times \delta$

$$
\begin{aligned}
& \frac{\partial h}{\partial \bar{z}_{i}}(z, w)-\frac{\partial \tilde{h}}{\partial \bar{z}_{i}}(z, \tilde{w}) \frac{\partial \varphi}{\partial w}(z, w) \\
& =\left\{\left[\left(\frac{\partial h}{\partial w} \frac{\partial \varphi}{\partial w}-h \frac{\partial^{2} \varphi}{\partial w^{2}}\right) \frac{\partial \varphi}{\partial \bar{z}_{i}}+\frac{\partial \varphi}{\partial w} h \frac{\partial^{2} \varphi}{\partial \bar{z}_{i} \partial w}\right] /\left(\frac{\partial \varphi}{\partial w}\right)^{2}\right\}_{(z, w)} \\
& =\left\{\frac{\partial}{\partial w}\left(\frac{\partial \varphi}{\partial \bar{z}_{i}} h / \frac{\partial \varphi}{\partial w}\right)\right\}_{(z, w)}
\end{aligned}
$$

Ce n'est autre que la formule (9.2) pour $(z, w) \in \Delta_{0} \times \delta$. Le lemme 4 est prouvé.

On donne ici une définition de la condition suivante $\left(L^{*}\right)$ plus faible que celle $(L)$ de $\S 1$ pour le triple $(\mathfrak{D}, \Delta, \pi)$.

Condition $\left(L^{*}\right)$. Il existe un triple $(\hat{D}, \Delta, \pi)$ tel que

(L1) $\mathfrak{D} \supset \mathfrak{D}$ et $\mathfrak{D}(z) \supset \mathfrak{D}(z)$ pour tout $z$ dans $\Delta$;

$\left(L^{*} 2\right)$ la frontière $\partial \mathfrak{D}$ de $\mathfrak{D}$ dans $\mathfrak{D}$ soit analytique au sens réel et $\mathfrak{S}$ soit pseudoconvexe (cf. p. 175 de K. Oka [10]) dans $\mathfrak{D}$ au sens suivant: soit donné un point $Q_{0}$ sur $\partial$ D tel que $\pi\left(Q_{0}\right)=z_{0} \in \Delta$. Alors il existe une famille de sections analytiques $\left\{\boldsymbol{E}_{t}(\cdot)\right\}_{0 \leqq t \leqq 1}$ de $\hat{\mathfrak{D}}$ sur $\Delta_{0}$ où $\Delta_{0}$ est un voisinage de $z_{0}$ dans $\Delta$ telle que (1) $\Xi_{t}(\cdot)$ soit continue pour $0 \leqq t \leqq 1$; (2) $\Xi_{0}\left(z_{0}\right)=Q_{0}$ et $\Xi_{0}\left(\Delta_{0}\right) \cap \mathfrak{D}=\phi$; (3) $\Xi_{t}\left(\Delta_{0}\right) \cap(D \cup \partial D)=\phi$ pour $0<t \leqq 1$.

Nous nous bornons désomais à deux triples $(\mathfrak{D}, \Delta, \pi)$ et $(\mathfrak{D}, \Delta, \tilde{\pi})$ satisfaisant à la

Condition $(E) . \quad(E 1)(\mathfrak{D}, \Delta, \pi)($ resp. $(\mathfrak{D}, \Delta, \tilde{\pi}))$ satisfasse aux conditions (L) (resp. $\left.\left(L^{*}\right)\right)$ et $(T)$; 
(E2) il existe un difféomorphisme $\varphi$ de classe $C^{\infty}$ de $\mathfrak{D}$ sur $\mathfrak{D}$ tel que $\tilde{\pi} \circ \varphi$ $=\pi$ et la restriction $\varphi(z, w)$ de $\varphi$ sur chaque $\mathfrak{D}(z)$ où $z \in \Delta$ soit analytique pour $w \in \mathfrak{D}(z)$;

(E3) $\chi=2 p+q-1 \geqq 2$ où $(p, q)$ est de type topologique de $\mathfrak{D}$.

D'après $(E 2)$ et $(E 3)$, l'argument de $\S 7$ entraîne qu'il existe les 1-formes holomorphes $\left\{\Omega_{k}(z, \cdot)\right\}$ (resp. $\left.\left\{\widetilde{\Omega}_{k}(z, \cdot)\right\}\right)(k=1, \cdots, \chi)$ sur $\mathfrak{D}(z)($ resp. $\mathfrak{D}(z))$ telles qu'ils soient linéairement indépendantes et que $\Omega_{k}(z, \cdot)=\varphi(z, \cdot)^{\sharp} \widetilde{\Omega}_{k}(z, \cdot)$. Pour $\chi$ nombres réels constants $\left\{x_{k}\right\}$, on pose

$$
\Omega(z, \cdot)=\sum_{k=1}^{\chi} x_{k} \Omega_{k}(z, \cdot) ; \quad \tilde{\Omega}(z, \cdot)=\sum_{k=1}^{\chi} x_{k} \widetilde{\Omega}_{k}(z, \cdot) .
$$

où $\operatorname{Re} \Omega_{k}(z, \cdot)(\operatorname{resp} . \tilde{\Omega}(z, \cdot))=\sum_{k=1}^{x} x_{k} \sigma_{k}(z, \cdot)$ (resp. $\left.\tilde{\sigma}_{k}(z, \cdot)\right)$ est de la forme (7.1). Par (E2), il vient pour $z \in \Delta$,

$$
\|\Omega(z, \cdot)\|_{D(z)}^{2}=\|\tilde{\Omega}(z, \cdot)\|_{\tilde{D}(z)}^{2},
$$

d'où pour $z \in \Delta$ et $i=1, \cdots, n$

$$
\frac{\partial^{2}}{\partial z_{i} \partial \bar{z}_{i}}\|\Omega(z, \cdot)\|_{Ф(z)}^{2}=\frac{\partial^{2}}{\partial z_{i} \partial \bar{z}_{i}}\|\tilde{\Omega}(z, \cdot)\|_{\tilde{D}(z)}^{2} \cdot
$$

Grâce à la condition ( $E 1)$, la formule 5 et l'inégalité $\left(4^{\prime}\right)$ de p. 508 de [15] entraînent l'inégalité fondamentale

$$
\left\|\frac{\partial \Omega(z, \cdot)}{\partial \bar{z}_{i}}\right\|_{\triangleright(z)}^{2} \geqq\left\|\frac{\partial \tilde{Q}(z, \cdot)}{\partial \bar{z}_{i}}\right\|_{\tilde{\Phi}(z)}^{2} .
$$

Par l'expression (9.2) et (9.3') il vient

$$
\begin{aligned}
& 2 \operatorname{Re}\left\{\left(\frac{\partial \Omega(z, \cdot)}{\partial \bar{z}_{i}}, \frac{\partial F(z, w)}{\partial w} d w\right)_{D(z)}\right\} \geqq\left\|\frac{\partial F(z, w)}{\partial w} d w\right\|_{\triangleright(z)}^{2} ; \\
& F(z, w)=\frac{\partial \varphi(z, w)}{\partial \bar{z}_{i}} \Omega(z, w) / \frac{\partial \varphi(z, w)}{\partial w} d w .
\end{aligned}
$$

L'inégalité de Schwarz et la formule 5 entraînent que

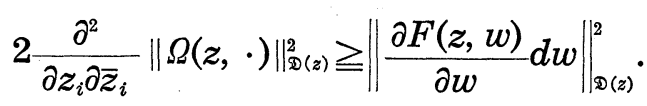

D’après cela, on a le

CorollaIre 5. Si (D, $\Delta, \pi)$ est trivial, la condition $(E)$ entraîne que $\varphi$ est isomorphisme analytique pour tout $(\mathfrak{D}, \Delta, \tilde{\pi})$.

En effet, puisque $(\mathfrak{D}, \Delta, \pi)$ est trivial, chaque $\Omega(z, \cdot)$ de la forme (9.3) ne 
dépend pas de $z \in \Delta$, d'où la premier membre de (9.5) est nulle pour tout $z \in \Delta$. Il s'ensuit d'après (9.5) que $F(z, w)$ est une constante $k(z, \Omega)$ sur $\mathfrak{D}(z)$. Par $(E 3)$, il existe au moins deux $\Omega_{j}(z, \cdot)(j=1,2)$ de la forme (9.3) linéairement indépendantes. Il en vient

$$
\left(-k\left(z, \Omega_{2}\right) \Omega_{1}(z, \cdot)+k\left(z, \Omega_{1}\right) \Omega_{2}(z, \cdot)\right) \cdot\left(\frac{\partial \varphi}{\partial \bar{z}_{i}} / \frac{\partial \varphi}{\partial w} d w\right)=0
$$

d'où que $\partial \varphi / \partial \bar{z}_{i}=0(i=1, \cdots, n)$ sur $\mathfrak{D}(z)$. Le corollaire est prouvé.

On montre un autre exemple à lequel l'inégalité (9.5) est appliquée. On a traité dans [13] le triple particulier comme suit: soit $\Delta$ un polydisque dans l'espace $\left(z_{1}, \cdots, z_{n}\right)$ et soit $\mathfrak{D}(z)$ où $z \in \Delta$ un ouvert connexe multivalent étalé au-dessus du plan $w$ tel que chaque composante frontière $C_{j}(j=1, \cdots, q)$ de $\mathfrak{D}(z)$ ne se varie pas avec $z \in \Delta: \partial \mathfrak{D}(z)=\bigcup_{j=1}^{q} C_{j}$, et que chaque point de ramification $\xi_{\nu}(z)(\nu=1, \cdots, m)$ de $\mathfrak{D}(z)$ soit analytique pour $z \in \Delta$ et $\xi_{\nu}(z) \neq$ $\xi_{\nu^{\prime}}(z)$ pour $\nu \neq \nu^{\prime}$. On a ainsi un triple $(\mathfrak{D}, \Delta, \pi)$ où $\pi^{-1}(z)=\mathfrak{D}(z)$ satisfaisant aux conditions $(L),(T)$. Pour simplicité on appelle tel triple, de type $(F)$. On pose $(p, q)$ le type topologique de $\mathfrak{D}$, et $\chi=2 p+q-1$. On écrit $\mu_{\nu}-1(\geqq 1)$ l'ordre de ramification de $\mathfrak{S}(z)$ au point $\xi_{\nu}(z)$. On considère une 1-forme $\Omega(z, \cdot)$ de la forme (9.3) telle que $\operatorname{Re} \Omega(z, \cdot)=\sum_{k=1}^{x} x_{k} \sigma_{k}(z, \cdot)$. Soit $\zeta_{0} \in C_{q}$ et formons sur chaque $\mathfrak{D}(z)$ où $z \in \Delta$ la fonction harmonique multiforme $v(z, w)$ $=\sum_{k=1}^{x} x_{k} v_{k}(z, w)$ telle que $d v_{k}(z, w)=\sigma_{k}(z, w)$ et $v_{k}\left(z, \zeta_{0}\right)=0$. D'après la preuve de la formule $5, \partial v(z, w) / \partial z_{i}(i=1, \cdots, n)$ est uniforme sur $\mathfrak{D}(z)$ et $v(z, w)$ est une constante $k_{j}$ sur $C_{j}(j=1, \cdots, q)$ de $\partial \mathfrak{D}(z)$ telle que $k_{j}$ ne dépende pas de $z \in \Delta$. Dans ce cas où $C_{j}$ ne se varie pas avec $z \in \Delta$, on a $\partial v(z, w) / \partial z_{i}=0$ sur $\partial \supseteqq(z)$. Mais $\partial v(z, w) / \partial z_{i}$ peut avoir des singularités au point $\xi_{\nu}(z)(\nu=1, \cdots, m)$. Dans un voisinage de $\xi_{\nu}(z)$, on a, d'après l'analycité de $\xi_{\nu}(z)$,

$$
\begin{aligned}
v(z, w)=\operatorname{Re} & \left\{\sum_{j=0}^{\infty} A_{j}(z)\left(w-\xi_{\nu}(z)\right)^{j / \mu_{\nu}}\right\} ; \\
\frac{\partial v}{\partial z_{i}}(z, w)= & -\frac{\left(\partial \xi_{\nu} / \partial z_{i}\right)(z)}{2 \mu_{\nu}} \sum_{l=1}^{\mu_{\nu}-1}\left(\mu_{\nu}-1\right) A_{\mu_{\nu}-1}(z)\left(w-\xi_{\nu}(z)\right)^{-l / \mu_{\nu}} \\
& +(\text { le terme borné). }
\end{aligned}
$$

Il s'ensuit que $\partial v / \partial z_{i}$ est régulière au point $\xi_{\nu}(z)$ si et seulement si, ou bien $\left(\partial \xi_{\nu} / \partial z_{i}\right)(z)=0$, ou bien $A_{1}(z)=\cdots=A_{\mu_{\nu}-1}(z)=0$, i.e., $(\partial v / \partial w) d w(=(1 / 2) \Omega(z, \cdot))$ y est zéro au moins d'ordre $\mu_{\nu}-1$. On en fait la

Remarque 6. Soit $(\mathfrak{D}, \Delta, \pi)$ un triple de type $(F)$. Soit $z_{0} \in \Delta$ et $\Omega(z, \cdot)$ où $z \in \Delta$ de la forme (9.3). Alors $\left\{\partial \Omega(z, \cdot) / \partial \bar{z}_{i}\right\}_{z=z_{0}}=0(i=1, \cdots, n)$ sur $\mathfrak{D}\left(z_{0}\right)$, si est seulement si, pour chaque point $\xi_{j}\left(z_{0}\right)$ de ramification de $\mathfrak{S}\left(z_{0}\right)$ d'ordre $\mu_{j}-1$ tel que $\left(\partial \xi_{j} / \partial z_{1}, \cdots, \partial \xi_{j} / \partial z_{n}\right)_{z=z_{0}} \neq(0, \cdots, 0)$, la 1 -forme holomorphe $\Omega\left(z_{0}, \cdot\right)$ sur $\mathfrak{D}\left(z_{0}\right)$ est zéro à $\xi_{j}\left(z_{0}\right)$ au moins d'ordre $\mu_{j}-1$. 
En effet, supposons que $\left\{\partial \Omega(z, \cdot) / \partial \bar{z}_{i}\right\}_{z=z_{0}}=0$ sur $\mathscr{D}\left(z_{0}\right)$. Il vient $\left(\partial^{2} v / \partial z_{i} \partial \bar{w}\right)$ $\left(z_{0}, w\right)=0$ sur $\mathfrak{D}\left(z_{0}\right)$, d'où $\left(\partial v / \partial z_{i}\right)\left(z_{0}, w\right)$ est méromorphe pour $w \in \mathfrak{D}\left(z_{0}\right)$. Puisque $\left(\partial v / \partial z_{i}\right)\left(z_{0}, w\right)=0$ sur $\partial \Phi\left(z_{0}\right)$, il s'ensuit par le théorème d'identité que $\left(\partial v / \partial z_{i}\right)\left(z_{0}, w\right)=0$ sur $\mathfrak{D}\left(z_{0}\right)$. Par l'hypothèse que $\left(\partial \xi_{j} / \partial z_{i}\right)(z) \neq 0$ pour certain $i$, $\Omega\left(z_{0}, \cdot\right)$ est zéro à $\xi_{j}\left(z_{0}\right)$ au moins d'ordre $\mu_{j}-1$. Dans le cas réciproque, $\left(\partial v / \partial z_{i}\right)\left(z_{0}, w\right)$ est régulière dans tout l'ouvert $\mathscr{D}\left(z_{0}\right)$. Puisque $\left(\partial v / \partial z_{i}\right)\left(z_{0}, w\right)=0$ $\operatorname{sur} \partial \mathfrak{D}\left(z_{0}\right)$, il vient par le théorème de maximum pour les fonctions harmoniques $\left(\partial v / \partial z_{i}\right)\left(z_{0}, w\right)=0$ dans $\mathfrak{D}\left(z_{0}\right)$, d'où $\left\{\partial \Omega(z, \cdot) / \partial \bar{z}_{i}\right\}_{z=z_{0}}=0$ sur $\mathfrak{D}\left(z_{0}\right)$.

Maintenant, on dénote par $M$ la somme des ordres $\mu_{j}-1$ de ramification de $\mathfrak{D}(z)$ pour tous les points de ramification non-constants $\xi_{j}(z)$ pour $z \in \Delta$. On écrit $K_{j}=\left\{z \in \Delta:\left(\partial \xi_{j} / \partial z_{i}\right)(z)=0(i=1, \cdots, n)\right\}$, qui est un ensemble analytique à dimension $n-1$ au plus dans $\Delta$. Puisque toute 1-forme non-zéro $\Omega(z, \cdot)$ de la forme $(9.3)$ a au plus $\chi-1$ zéros sur $\mathscr{D}(z)$, il en résulte d'après la remarque 6 que dans le cas où $M \geqq \chi$, pour tout $z_{0} \in \Delta-\bigcup_{j} K_{j}$ et toute $\Omega(z, \cdot)$ non-zéro de la forme (9.3), $\left\{\partial \Omega(z, \cdot) / \partial \bar{z}_{i}\right\}_{z=z_{0}}$ n'est pas nulle sur $\mathscr{D}\left(z_{0}\right)$, d'où, par

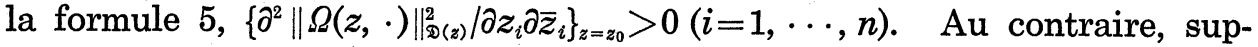
posons que $\chi \geqq 2(M+1)$. Soit $z_{0}$ fixé dans $\Delta-\bigcup_{j} K_{j}$. On trouve alors aisément deux éléments $\omega_{l}(w)(l=1,2)$ dans $\operatorname{Re} \Gamma_{h 0}\left(\mathfrak{D}\left(z_{0}\right)\right)=\left\{\sum_{k=1}^{x} x_{k} \sigma_{k}(w): x_{k}\right.$ est réel constant $\}$ tels que $\left\{\omega_{l}(w)\right\}_{l=1,2}$ soient linéairement indépendants et que $\omega_{l}(w)+\sqrt{-1} \omega_{l}^{*}(w)$ soit zéros au moins d'ordre $\mu_{j}-1$ à chaque $\xi_{j}\left(z_{0}\right)$. On peut former $\Omega_{l}(z, \cdot)$ sur $\mathfrak{D}(z)$ où $z \in \Delta$ de la forme (9.3) telle que $\Omega_{l}(z, \cdot)=\omega_{l}+\sqrt{-1} \omega_{l}^{*}$ sur $\mathfrak{D}\left(z_{0}\right)$. Par la remarque 6 on a $\left\{\partial \Omega_{l}(z, \cdot) / \partial \bar{z}_{i}\right\}_{z=z_{0}}=0(i=1, \cdots, n)$, d'où par

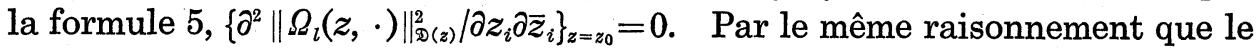
corollaire 5 , on a $\left(\partial \varphi(z, w) / \partial \bar{z}_{i}\right)\left(z_{0}, w\right)=0$ pour $w \in \mathfrak{D}\left(z_{0}\right)$. $z_{0}$ étant quelconque, $\varphi(z, w)$ citée en (E2) est analytique pour $z \in \Delta$. On a obtenu le

ThÉonème 3. Pour le triple $(\mathfrak{D}, \Delta, \pi)$ de type $(F)$ tel que $\chi \geqq 2(M+1)$, la condition $(E)$ entraîne l'isomorphisme analytique de $\varphi$ pour tout $(\tilde{D}, \Delta, \tilde{\pi})$.

Les exemples suivants nous montrent que le théorème 3 n'est pas trivial. Pas définition on a les inclusions entre les types de triples: (trivials) $\subsetneq(F) \subsetneq$ $(L) \subseteq\left(L^{*}\right)$.

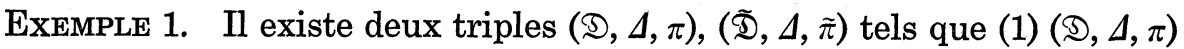
soit de type $(F)$ et satisfasse à la condition $(T)$ de type topologique $(p, q)$ où $\chi=2 p+q-1 \geqq 2 ;(2)(\tilde{D}, \Delta, \tilde{\pi})$ satisfasse aux conditions $\left(L^{*}\right),(T)$ mais non à celle $(L)$; (3) pour tout $z \in \Delta, \mathfrak{D}(z)$ et $\mathfrak{D}(z)$ soient équivalentes comme surface de Riemann. Pour cela, soit $\Delta=\{|z-1 / 2|<1 / 2\}$. Pour $z$ fixé dans $\Delta$, considérons la transformation conforme suivante $\varphi(z, w)$ du cercle $U=\{|w|<1\}$ sur celui $D_{1}(z)=\{|w|<R(z)\}: \varphi(z, w)=[(w-z) /(1-\bar{z} w)]\left[1-\left(|z|^{2} / 2\right)\right] /(-z / 2)$ de façon que $\varphi(z, z)=0, \varphi(z, z / 2)=1$ et $R(z)=\left[1-\left(|z|^{2} / 2\right)\right] /(|z| / 2)$. On a $\partial^{2} \log R(z) /$ 
$\partial z \partial \bar{z}=(-1 / 2) /\left[1-\left(|z|^{2} / 2\right)\right]^{2}<0$ pour $z \in \Delta$. Il s'ensuit grâce à $F$. Hartogs que l'ouvert connexe $D_{1}=\bigcup_{z \in \Delta}\left(z, D_{1}(z)\right)$ dans le dicylindre $\Delta \times\{|W|<\infty\}$ est strictement pseudoconvexe. On écrit $\mathfrak{D}$ (resp. $\mathfrak{D})$ l'ouvert connexe étalé en nombre $N(\geqq 3)$ au-dessus de $\Delta \times U$ (resp. $D_{1}$ ) sans point frontière relatif tel que les surfaces de ramification soient $\{w=z, w=z / 2\}$ (resp. $\{W=0, W=1\}$ ) d'ordre $N-1$. Quand on considère le triple $(\mathfrak{D}, \Delta, \pi)(\operatorname{resp} .(\mathfrak{D}, \Delta, \tilde{\pi}))$ où $\pi$ (resp. $\tilde{\pi})$ est la projection de $\mathfrak{D}($ resp. $\mathscr{D}) \operatorname{sur} \Delta$, alors $(\mathfrak{D}, \Delta, \pi)$ et $(\tilde{D}, \Delta, \tilde{\pi})$ satisfont évidemment aux conditions demandées pour $(p, q)=(0, N)$.

Exemple 2. Il existe deux triples $(\mathfrak{D}, \Delta, \pi),(\mathfrak{D}, \Delta, \tilde{\pi})$ tels que $(1) \mathfrak{D}$ et $\mathfrak{D}$ soient de type $(F)$ et vérifient à la condition $(T)$ de type $(p, q)$ où $\chi=2 p+q-$ $1 \geqq 2 ;(2)(\mathfrak{D}, \Delta, \pi)$ et $(\tilde{D}, \Delta, \tilde{\pi})$ ne soient pas équivalents; (3) pour tout $z \in \Delta$, $\mathfrak{D}(z)$ et $\mathfrak{D}(z)$ soient équivalentes comme surface de Riemann. Pour cela, soit $\Delta=\{|z|<1\}$ et $f(z)$ une fonction holomorphe non-constante pour $z \in \Delta$ telle que $0<|f(z)|<1$. Soit $m(\geqq 1)$ un nombre entier. On considère la partie $\mathfrak{H}_{m}$ étalée au-dessus du dicylindre $\Delta \times U$ où $U=\{|w|<1\}$ de la surface de Riemann de

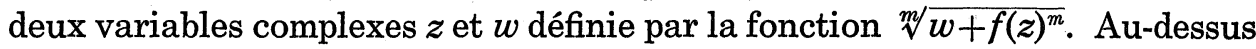
de la droite $\{w=0\}$ dans $\Delta \times U$, on trouve $m$ droites $\left\{W=O^{(i)}\right\}_{i=1, \ldots, m}$ de $\mathfrak{U}_{m}$. On forme la transformation de $\mathfrak{U}_{m}$ sur $\Delta \times V$ où $V=\{|W|<1\}:(z, w) \rightarrow(z, W)$ où $W=\psi_{m}(z, w)=w_{2} \circ w_{1}(z, w) ; w_{1}(z, w)=\left(w+f(z)^{m}\right) /\left(1+\overline{f(z)^{m}} w\right) ; w_{2}=\sqrt[m]{w_{1}}$ de façon que $\psi_{m}\left(z, O^{(1)}\right)=f(z), \psi_{m}\left(z,-f(z)^{m}\right)=0$ et $\psi_{m}(z, w)$ transforme conformément et bijectivement chaque fibre $\mathfrak{U}_{m}(z)\left(=\mathfrak{U}_{m} \cap\{z=z\}\right)$ où $z \in \Delta$ sur $V$. On écrit $\mathfrak{D}_{m}$ (resp. $\left.\mathfrak{D}_{0}\right)$ l'ouvert connexe étalé en nombre $N(\geqq 3)$ au-dessus de $\mathfrak{U}_{m}$ (resp. $\Delta \times V$ ) sans point frontière relatif tel que les surfaces de ramification soient $\left\{w=O^{(1)}, w=-f(z)^{m}\right\}$ (resp. $\{W=f(z), W=0\}$ ) d'ordre $N-1$. Quand on pose $\pi_{m}$ la projection de $\mathfrak{D}_{m}$ sur $\Delta$, on obtient des triples $\left\{\left(\mathscr{D}_{m}, \Delta, \pi_{m}\right)\right\}_{m=0,1,2, \ldots}$. Alors deux triples quelconques $\left(\mathfrak{S}_{m}, \Delta, \pi_{m}\right),\left(\mathfrak{S}_{l}, \Delta, \pi_{l}\right)$ où $m-1>l \geqq 0$ satisfont évidemment aux conditions (1), (3). Pour prouver (2), supposons qu'il existe un isomorphisme analytique $\varphi$ de $\mathfrak{D}_{m}$ sur $\mathfrak{D}_{l}$ tel que $\pi_{l} \circ \varphi=\pi_{m}$. Puisque $\mathfrak{D}_{m}$ et $\mathfrak{D}_{l}$ sont de type $(F)$ et $f(z)$ n'est pas constante, il s'ensuit d'après (3) du lemme 2 de $\S 4$ que $\varphi\left(z,-f(z)^{m}\right)=-f(z)^{l}$ (resp. $f(z)$ où $l=0$ ) et l'ordre de ramification de $\mathfrak{D}_{m}$ comme revêtement au-dessus du dicylindre $\Delta \times U$ (resp. $\Delta \times V$ ) le long de $w=-f(z)^{m}$ est égale à celui de $\mathfrak{D}_{l}$ le long de $w=-f(z)^{l}$ (resp. $W=$ $f(z)$ ). Mais par construction ces ordres sont $m N-1$ et $l N-1$ (resp. $N-1$ ) respectivement, qui est contradiction.

ExEmple 3. Il existe deux triples (D্, $\Delta, \pi),(\mathfrak{D}, \Delta, \tilde{\pi})$ tels que (1) la même condition que (1) de l'exemple $2 ;(2)(\mathfrak{D}, \Delta, \pi)$ et $(\mathfrak{D}, \Delta, \tilde{\pi})$ soient équivalents; (3) il existe une transformation non-analytique $\varphi$ de $\mathfrak{D}$ sur $\mathfrak{D}$ telle que $\tilde{\pi} \circ \varphi$ $=\pi$ et la restriction de $\varphi$ sur chaque $\mathfrak{D}(z)$ où $z \in \Delta$ soit analytique. Pour cela, il suffit de prendre dans l'exemple 2 , $\mathfrak{D}=\mathfrak{D}_{1}, \mathfrak{D}=\mathfrak{D}_{0}$ et $\varphi:(z, w) \rightarrow$ $\left(z, \psi_{1}(z, w)\right)$. 
On va montrer une condition générale (voir le théorème 4) pour que deux triples soient équivalents. On commence par transformer l'expression (9.4).

Soient $(D, \Delta, p),(\tilde{D}, \Delta, \tilde{p})$ deux triples satisfaisant à la

Condition $\left(E^{\prime}\right)$. $\quad D$ et $\tilde{D}$ sont ouverts connexes multivalents en nombre fini étalé au-dessus du polycylindre $\Delta \times C$ ( $\Delta$ étant un polydisque de centre l'origine dans l'espace $\left(z_{1}, \cdots, z_{n}\right)$ et $\left.C=\{|w|<\infty\}\right)$ avec frontières analytiques au sens réel dans $\Delta \times C$ tels que

$\left(E^{\prime} 0\right) \quad D$ (resp. $\left.\tilde{D}\right)$ n'ait aucune surface de ramification et $p$ (resp. $\left.\tilde{p}\right)$ désignant la projection de $D$ (resp. $\tilde{D})$ sur $\Delta$, l'ouvert connexe $p^{-1}(0)=D(0)$ audessus de $C$ soit exactement égale à celui $\tilde{p}^{-1}(0)=\tilde{D}(0)$. On en écrit $C(0)=$ $=\partial D(0)=\partial \tilde{D}(0)$;

$\left(E^{\prime} 1\right)(D, \Delta, p)(\operatorname{resp} .(\tilde{D}, \Delta, \tilde{p}))$ satisfasse aux conditions $(L)\left(\operatorname{resp} .\left(L^{*}\right)\right)$ et $(T)$. Il en vient que $\partial D(z)=\{\xi(z, \zeta): \zeta \in C(0)\}$ tel que $\xi(0, \zeta)=\zeta$ et $\xi(z, \zeta)$ soit, pour $\zeta$ fixé sur $C(0)$, fonction analytique pour $z \in \Delta$, et qu'il existe, pour $\zeta$ fixé sur $C(0)$, une fonction analytique $\eta(z, \zeta)$ pour $z \in \Delta_{0}\left(\Delta_{0}\right.$ étant un polydisque de centre l'origine dans $\Delta)$ telle que $\eta(0, \zeta)=\zeta$ et $\left\{(z, \eta(z, \zeta)): z \in \Delta_{0}\right\} \cap \tilde{D}=\phi$;

$\left(E^{\prime} 2\right)$ il existe un difféomorphisme $\varphi$ de $D$ sur $\tilde{D}$ tel que la restriction $\varphi(z, w)$ de $\varphi$ sur chaque fibre $D(z)$ où $z \in \Delta$ soit analytique pour $w \in D(z)$ et $\varphi(0, w)=w$ sur $D(0)$

( $\left.E^{\prime} 3\right)$ le type topologique de $D$ est $(p, q)$ tel que $q \geqq 2$.

D'après $\left(E^{\prime} 3\right)$, il existe sur chaque $D(z)$ (resp. $\tilde{D}(z)$ ) une fonction harmonique uniforme réelle non-constante $u(z, w)$ (resp. $\tilde{u}(z, w)$ ) telle que $u(z, \zeta)$ $($ resp. $\tilde{u}(z, \zeta))=1$ sur $C_{1}(z)\left(\right.$ resp. $\left.\tilde{C}_{1}(z)\right)$ et $=0$ sur $\partial D(z)-C_{1}(z)(\operatorname{resp} . \partial \tilde{D}(z)-$ $\left.\tilde{C}_{1}(z)\right)$, où $C_{1}(z)\left(\operatorname{resp} . \tilde{C}_{1}(z)\right)$ est une composante frontière de $D(z)($ resp. $\tilde{D}(z))$ telle que $\varphi\left(z, C_{1}(z)\right)=\tilde{C}_{1}(z)$ et $C_{1}(z)$ se varie continûment avec $z \in \Delta$. Si l'on pose $\Omega(z, \cdot)($ resp. $\tilde{\Omega}(z, \cdot))=2(\partial u / \partial w)(z, w)($ resp. $2(\partial \tilde{u} / \partial w)(z, w))$, ils sont de la forme (9.3) et, d'après $\left(E^{\prime} 2\right), \Omega(z, \cdot)=\varphi(z, \cdot)^{\sharp} \widetilde{\Omega}(z, \cdot)$. D'après $(9.4)$ pour $z=0$, on obtient, par $\varphi(0, w) / \partial w=1$, l'inégalité suivante

$$
\iint_{D(0)}\left|\frac{\partial}{\partial w}\left(\frac{\partial \varphi}{\partial \bar{z}_{i}} \frac{\partial u}{\partial w}\right)\right|_{(0, w)}^{2} d u d v \leqq 2 \operatorname{Re} \iint_{D(0)}\left\{\overline{\frac{\partial}{\partial w}\left(\frac{\partial \varphi}{\partial \bar{z}_{i}} \frac{\partial u}{\partial w}\right)} \frac{\partial^{2} u}{\partial \bar{z}_{i} \partial w}\right\}_{(0, w)} d u d v
$$

Grâce à la formule de Stokes il vient par $E^{\prime}(0)$

$$
=\operatorname{Im} \int_{C(0)}\left\{\left(\overline{\frac{\partial \varphi}{\partial \bar{z}_{i}} \frac{\partial u}{\partial w}}\right) \frac{\partial^{2} u}{\partial \bar{z}_{i} \partial w}\right\}_{(0, w)} d w
$$

D'après $\left(E^{\prime} 2\right)$, il vient $\tilde{u}(z, \varphi(z, w))=u(z, w)$ pour $(z, w) \in D \cup \partial D$. En posant $w=\xi(z, \zeta)$ pour $z \in \Delta$ et $\zeta \in C_{1}(0)\left(\right.$ resp. $\left.\zeta \in C(0)-C_{1}(0)\right)$, on a

$$
\tilde{u}(z, \varphi(z, \xi(z, \zeta)))=u(z, \xi(z, \zeta))=0(\text { resp. }=1) \text {. }
$$

Par $z_{i}$-différentelle, il vient d'après $\left(E^{\prime} 1\right)$, pour $(z, \zeta) \in \Delta \times C(0)$ 


$$
\begin{aligned}
& \frac{\partial \tilde{u}}{\partial z_{i}}(z, \varphi(z, \xi(z, \zeta)))+\frac{\partial \tilde{u}}{\partial w}(z, \varphi(z, \xi(z, \zeta)))\left\{\frac{\partial \varphi}{\partial z_{i}}(z, \xi(z, \zeta))+\frac{\partial \varphi}{\partial w}(z, \xi(z, \zeta)) \frac{\partial \xi}{\partial z_{i}}(z, \zeta)\right\} \\
& \quad+\frac{\partial \tilde{u}}{\partial \bar{w}}(z, \varphi(z, \xi(z, \zeta))) \frac{\partial \bar{\varphi}}{\partial z_{i}}(z, \xi(z, \zeta))=0 .
\end{aligned}
$$

On pose $z=0$, et $\left(E^{\prime} 0\right),\left(E^{\prime} 1\right),\left(E^{\prime} 2\right)$ entraînent que pour $\zeta \in C(0)$

$$
\left\{\frac{\partial \tilde{u}}{\partial z_{i}}+\frac{\partial u}{\partial w}\left(\frac{\partial \varphi}{\partial z_{i}}+\frac{\partial \xi}{\partial z_{i}}\right)+\frac{\partial u}{\partial \bar{w}} \frac{\partial \bar{\varphi}}{\partial z_{i}}\right\}_{(0, \zeta)}=0
$$

D’après $\left(E^{\prime} 1\right)$, on a pour $\zeta \in C_{1}(0)\left(\right.$ resp. $\left.C(0)-C_{1}(0)\right), \tilde{u}(z, \eta(z, \zeta)) \geqq 1($ resp. $\leqq 0)$ pour $z \in \Delta_{0}$ et l'égalité est vraie pour $z=0$. Il en vient pour $\zeta \in C(0)$

$$
\left\{\frac{\partial \tilde{u}}{\partial z_{i}}+\frac{\partial u}{\partial w} \frac{\partial \eta}{\partial z_{i}}\right\}_{(0, \zeta)}=0
$$

On en a pour $\zeta \in C(0)$

$$
\left\{\overline{\frac{\partial u}{\partial w} \frac{\partial \varphi}{\partial \bar{z}_{i}}}\right\}_{(0, \zeta)}=\left\{\frac{\partial u}{\partial w}\left(\frac{\partial \eta}{\partial z_{i}}-\frac{\partial \xi}{\partial z_{i}}\right)-\frac{\partial u}{\partial w} \frac{\partial \varphi}{\partial z_{i}}\right\}_{(0, \zeta)} .
$$

Puisque, par $\left(E^{\prime} 0\right)$, chaque $D(z)$ n'a pas de point de ramification, $(\partial u / \partial w)(0, w)$, $\left(\partial \varphi / \partial z_{i}\right)(0, w)$ et $\left(\partial^{2} u / \partial \bar{z}_{i} \partial w\right)(0, w)$ sont fonctions holomorphes et uniformes pour $w \in D(0)$. Grâce au théorème de Cauchy, on a

$$
\int_{C(0)}\left\{\frac{\partial u}{\partial w} \frac{\partial \varphi}{\partial z_{i}} \frac{\partial^{2} u}{\partial \bar{z}_{i} \partial w}\right\}_{(0, w)} d w=0
$$

Il s'ensuit par (9.6), (9.7) qu'on a, sous les conditions $\left(E^{\prime} 0\right) \sim\left(E^{\prime} 3\right)$,

$$
\iint_{D(0)}\left|\frac{\partial}{\partial w}\left(\frac{\partial \varphi}{\partial \bar{z}_{i}} \frac{\partial u}{\partial w}\right)\right|_{(0, w)}^{2} d u d v \leqq \operatorname{Im} \int_{C(0)}\left\{\frac{\partial u}{\partial w}\left(\frac{\partial \eta}{\partial z_{i}}-\frac{\partial \xi}{\partial z_{i}}\right) \frac{\partial^{2} u}{\partial \bar{z}_{i} \partial w}\right\}_{(0, w)} d w
$$

On donne deux définitions habituelles. Soit $(\mathfrak{D}, \Delta, \pi)$ un triple où $\Delta$ est un polydisque dans l'espace $\left(z_{1}, \cdots, z_{n}\right)$. Soit $\Delta_{n+1}=\left\{\left|z_{n+1}\right|<\rho_{n+1}\right\}$. Quand on pose $\mathfrak{D}^{(n+1)}=\mathfrak{D} \times \Delta_{n+1}, \Delta^{(n+1)}=\Delta \times \Delta_{n+1}$ et $\pi^{(n+1)}\left(\left(P, z_{n+1}\right)\right)=\left(\pi(P), z_{n+1}\right)$ pour tout $\left(P, z_{n+1}\right) \in \mathfrak{D}^{(n+1)}$, on obtient un triple $\left(\mathfrak{D}^{(n+1)}, \Delta^{(n+1)}, \pi^{(n+1)}\right)$ qui s'appellera le produit de $(\mathfrak{D}, 4, \pi)$ par $\Delta_{n+1}$. Supposons ensuite $n \geqq 2$. Pour l'ensemble analytique $K$ de $\Delta$, on pose $\mathfrak{D} \mid K=\pi^{-1}(K),(\pi \mid K)(P)=\pi(P)$ pour tout $P \in \mathfrak{D} \mid K$. On a ainsi un triple $(\mathfrak{D}|K, K, \pi| K)$ qui s'apellera la restriction de $(\mathfrak{D}, \Delta, \pi)$ sur $K$.

Les propriétés (1), (2), (3) des conditions $\left(L^{*}\right),(T)$ entraînent par le théorème de Hurwitz la remarque suivante qui servira à la preuve du théorème 4. 
Remarque 7. Soit $\Delta=\Delta^{(1)} \times \Delta^{(2)}, \Delta^{(i)}(i=1,2)$ étant polydisque dans l'espace $\left(z_{1}^{(i)}, \cdots, z_{n_{i}}^{(i)}\right)$. Soit $(\mathfrak{D}, \Delta, \pi)$ un triple satisfaisant aux conditions $\left(L^{*}\right),(T)$. Supposons que $(a)$ il existe un certain point $c$ de $\Delta^{(2)}$ tel que la restriction de $(\mathfrak{D}, \Delta, \pi)$ sur $\Delta^{(1)} \times\{c\}$ satisfasse à la condition $(L) ;(b)$ pour tout $z^{(1)}$ fixé dans $\Delta^{(1)}$, la restriction de $(\mathfrak{D}, \Delta, \pi)$ sur $\left\{z^{(1)}\right\} \times \Delta^{(2)}$ satisfasse à celle $(L)$. Alors le triple (D) $\Delta, \pi$ ) lui-même satisfait à la condition $(L)$.

Comme application de l'inégalité (9.8), on obtient un théorème d'homotopie analytique concernant les équivalences de variations analytiques comme suit.

THÉORÈME 4. Soit $\left(\mathfrak{D}, \Delta^{(n+1)}, \pi\right)$ un triple où $\Delta^{(n+1)}=\Delta \times \Delta_{n+1}, \Delta=\Delta_{1} \times \cdots$ $\times \Delta_{n}, \Delta_{i}=\left\{\left|z_{i}\right|<\rho_{i}\right\}(i=1, \cdots, n, n+1)$ et $n \geqq 1$. Supposons que

(1) (D, $\left.\Delta^{(n+1)}, \pi\right)$ satisfasse aux conditions $\left(L^{*}\right),(T)$;

(2) la restriction de $\left(\mathfrak{D}, \Delta^{(n+1)}, \pi\right)$ sur $\Delta \times\{0\}$, qu'on écrit en abrégé (D) $\Delta, \Delta, \pi \mid \Delta)$, satisfasse aux conditions $(L),(T)$;

(3) pour tout $\left(z, z_{n+1}\right) \in \Delta^{(n+1)}$ où $z \in \Delta$ et $z_{n+1} \in \Delta_{n+1}$, la fibre $\pi^{-1}\left(\left(z, z_{n+1}\right)\right)$ soit équivalente comme surface de Riemann à celle $\pi^{-1}((z, 0))$;

(4) $\chi=2 p+q-1 \geqq 2$ où $(p, q)$ est de type topologique de (פ, $\left.\Delta^{(n+1)}, \pi\right)$.

Alors le triple $\left(\mathfrak{D}, \Delta^{(n+1)}, \pi\right)$ est équivalent au produit de $(\mathfrak{D}|\Delta, \Delta, \pi| \Delta)$ par $\Delta_{n+1}$.

Démonstration. Pour $\left(z, z_{n+1}\right)$ fixé dans $\Delta \times \Delta_{n+1}$, on trouve d'après (3) une application conforme $\varphi\left(z, z_{n+1}, \cdot\right)$ de la fibre $\mathscr{D}\left(\left(z, z_{n+1}\right)\right)\left(=\pi^{-1}\left(\left(z, z_{n+1}\right)\right)\right)$ sur celle $\mathfrak{S}((z, 0))$ de façon que $\varphi(z, 0, \cdot)$ soit transformation identique et $\varphi\left(z, z_{n+1}, w\right)$ soit analytique au sens réel dans $\mathfrak{D}$. Il suffit de vérifier que $\varphi\left(z, z_{n+1}, w\right)$ est analytique par rapport à $\left(z, z_{n+1}\right) \in \Delta \times \Delta_{n+1}$. Sous notre situation, $z_{n+1}=0$ est le point particulier de $\Delta_{n+1}$, mais $z=0$ n'est pas de tel point de $\Delta$. Il suffit pour cela de prouver la relation

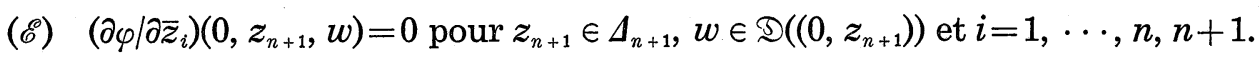

On peut supposer pour prouver $(\mathscr{E})$ que

$$
q \geqq 3 .
$$

Car si non, d'après (4) on a $p \geqq 1$. Il s'ensuit par la condition $(T)$ qu'il existe une surface $A$ de dimension réelle $2 n-1$ dans $\mathfrak{D}$ telle que, quand on pose $A(z)=\pi^{-1}(z)$ pour $z \in \Delta^{(n+1)}, A(z)$ soit une courbe fermée sur $\mathfrak{D}(z)$ telle que $\mathfrak{D}(z)$ $-A(z)$ soit connexe. Il suffit de former le revêtement triplement étalé audessus de $\mathfrak{D}$ le long de $A$ sans point frontière relatif ni surface de ramifi cation.

Or, par l'argument de $\S 2$ (si nécessaire, on reprend plus petit polydisque de l'origine que $\left.\Delta^{(n+1)}\right)$, one peut de plus supposer que $\left(\mathfrak{D}, \Delta^{(n+1)}, \pi\right)$ soit équivalent à $\left(D, \Delta^{(n+1)}, p\right)$ où $D(\subset U)$ est un ouvert connexe étalé au-dessus du 
polycylindre $\Delta^{(n+1)} \times C$ où $C=\{|w|<\infty\}$ et $p$ est la projection de $D$ sur $\Delta^{(n+1)}$ tel que

(D1) $D$ n'ait aucun surface de ramification;

(D2) $\partial D$ soit analytique au sens réel dans $U$ et chaque $\partial D(z)$ où $z \in \Delta^{(n+1)}$ consiste en $q$ courbes fermées sur $U(z)$.

L'application $\varphi\left(z, z_{n+1}, \cdot\right)$ ci-dessus nous conduit à celle conforme suivante, qu'on désigne par même notation $\varphi\left(z, z_{n+1}, \cdot\right)$ :

$$
\begin{gathered}
\varphi\left(z, z_{n+1}, \cdot\right): D\left(\left(z, z_{n+1}\right)\right) \longrightarrow D((z, 0)) \quad \text { pour }\left(z, z_{n+1}\right) \in \Delta \times \Delta_{n+1} \\
\varphi(z, 0, w)=w \quad \text { pour } z \in \Delta \text { et } w \in D((z, 0)) .
\end{gathered}
$$

On fixe $z_{0}$ dans $\Delta$ et considère la restriction du triple $\left(D, \Delta^{(n+1)}, p\right) \operatorname{sur}\left(z_{0}, \Delta_{n+1}\right)$, qu'on écrit simplement $\left(D\left|z_{0}, \Delta_{n+1}, p\right| z_{0}\right)$. D'après (1), (4), cette restriction satisfait aux conditions $\left(L^{*}\right),(T)$ tel que $\chi \geqq 2$. Quand on considère le triple trivial $\Delta_{n+1} \times D\left(\left(z_{0}, 0\right)\right)$ muni de la première projection, le corollaire 5 avec la condition (3) entraîne que $\varphi\left(z_{0}, z_{n+1}, w\right)$ est anlytique pour $z_{n+1} \in \Delta_{n+1}$. $z_{0}$ étant quelconque de $\Delta$, on a

$$
\frac{\partial \varphi}{\partial \bar{z}_{n+1}}=0 \quad \text { dans } D
$$

En particulier, pour tout $z_{0} \in \Delta$, le triple $\left(D\left|z_{0}, \Delta_{n+1}, p\right| z_{0}\right)$ satisfait à la condition $(L)$. Par les conditions (1), (2), il s'ensuit d'après la remarque 7 que le triple $\left(D, \Delta^{(n+1)}, p\right)$ satisfait à la condition $(L)$. On en sait que

(D3) pour $\left(z, z_{n+1}\right) \in \Delta \times \Delta_{n+1}$, il vienne $\partial D\left(\left(z, z_{n+1}\right)\right)=\left\{\xi\left(z, z_{n+1} ; \zeta\right): \zeta \epsilon\right.$ $\partial D((0,0))\}$ telle que $\xi(0,0 ; \zeta)=\zeta$ pour $\zeta \in \partial D((0,0))$, et $\xi\left(z, z_{n+1} ; \zeta\right)$ soit, pour $\zeta$ fixé sur $\partial D((0,0))$, analytique pour $\left(z, z_{n+1}\right) \in \Delta \times \Delta_{n+1}$.

Si l'on reprend par $(D 1),(D 2)$ plus petit polydisque de centre l'origine que $\Delta \times \Delta_{n+1}$, qu'on désigne par même notation $\Delta \times \Delta_{n+1}$, on peut supposer que

(D4) il existe un voisinage $V((0,0))$ de $D((0,0))$ dans $U((0,0))$ tel que $D\left(\left(z, z_{n+1}\right)\right) \subset V((0,0))$ pour tout $\left(z, z_{n+1}\right) \in \Delta \times \Delta_{n+1}$, et chaque $\varphi\left(z, z_{n+1}, w\right)$ où $\left(z, z_{n+1}\right) \in \Delta \times \Delta_{n+1}$ soit définie et holomorphe pour $w$ dans $V((0,0))$ et $(\partial \varphi / \partial w)$ $\left(z, z_{n+1}, w\right)$ ne s'annule à aucun $w \in V((0,0))$.

Maintenant on pose pour $\left(z, z_{n+1}\right) \in \Delta \times \Delta_{n+1}$ et $\zeta \in \partial D((0,0))$,

$$
\begin{aligned}
& \varphi\left(0, z_{n+1}, D\left(\left(z, z_{n+1}\right)\right)\right)=D^{*}\left(\left(z, z_{n+1}\right)\right) ; \\
& \varphi\left(0, z_{n+1}, \xi\left(z, z_{n+1} ; \zeta\right)\right)=\xi^{*}\left(z, z_{n+1} ; \zeta\right) .
\end{aligned}
$$

Par (9.10) il vient

$$
D^{*}((z, 0))=D((z, 0)) \quad \text { pour } z \in \Delta .
$$

En particulier, $D^{*}((0,0))=D((0,0))$, d'où $\partial D^{*}((0,0))=\partial D((0,0))$. D'après $(D 4)$ et la définition de $\varphi\left(z, z_{n+1}, \cdot\right)$, il s'ensuit que 
$\left(D^{*} 0\right)$ chaque $D^{*}\left(\left(z, z_{n+1}\right)\right)$ où $\left(z, z_{n+1}\right) \in \Delta \times \Delta_{n+1}$ est un ouvert connexe au-dessus du plan $w$ sans point de ramification, et $D^{*}\left(\left(0, z_{n+1}\right)\right)=D^{*}((0,0))$ pour tout $z_{n+1} \in \Delta_{n+1}$.

Par (D3) et (9.10), il vient

$\left(D^{*} 1\right) \quad \xi^{*}(0,0 ; \zeta)=\zeta$ pour $\zeta \in \partial D^{*}((0,0))$, et $\partial D^{*}\left(\left(z, z_{n+1}\right)\right)=\left\{\xi^{*}\left(z, z_{n+1} ; \zeta\right)\right.$ : $\left.\zeta \in \partial D^{*}((0,0))\right\}$ pour $\left(z, z_{n+1}\right) \in \Delta \times \Delta_{n+1}$.

Grâce à l'égalité (9.11) et $(D 3), \xi^{*}\left(z, z_{n+1} ; \zeta\right)$ est, pour $\zeta$ fixé sur $\partial D^{*}((0,0))$, analytique par rapport à $\left(z, z_{n+1}\right) \in \Delta \times \Delta_{n+1}$. On a ainsi un triple $\left(D^{*}, \Delta \times\right.$ $\left.\Delta_{n+1}, p^{*}\right)$ défini par $p^{*-1}\left(\left(z, z_{n+1}\right)\right)=D^{*}\left(\left(z, z_{n+1}\right)\right)$ satisfait aux conditions $(L),(T)$ tel que le triple $\left(D, \Delta \times \Delta_{n+1}, p\right)$ soit équivalent à celui $\left(D^{*}, \Delta \times \Delta_{n+1}, p^{*}\right)$ par l'isomorphisme analytique $\left(z, z_{n+1}, w\right) \rightarrow\left(z, z_{n+1}, \varphi\left(0, z_{n+1}, w\right)\right)$. Pour $\left(z, z_{n+1}\right)$ fixé dans $\Delta \times \Delta_{n+1}$, on désigne par $\varphi^{-1}\left(z, z_{n+1}, \cdot\right): D((z, 0)) \rightarrow D\left(\left(z, z_{n+1}\right)\right)$ la transformation inverse de $\varphi\left(z, z_{n+1}, \cdot\right)$, et considère une application $\psi\left(z, z_{n+1}, \cdot\right)$ définie par $\psi\left(z, z_{n+1}, \cdot\right)=\varphi\left(0, z_{n+1}, \varphi^{-1}\left(z, z_{n+1}, \cdot\right)\right)$ ou $\psi\left(z, z_{n+1}, \varphi\left(z, z_{n+1}, w\right)\right)=$ $\varphi\left(0, z_{n+1}, w\right)$ pour $w \in V((0,0))$. Il est évident par (9.12) et la définition $D^{*}\left(\left(z, z_{n+1}\right)\right)$ que

$\left(D^{*} 2\right)$ elle est une application conforme telle que

$$
\begin{aligned}
& \psi\left(z, z_{n+1}, \cdot\right): D^{*}((z, 0)) \longrightarrow D^{*}\left(\left(z, z_{n+1}\right)\right) ; \\
& \psi\left(0, z_{n+1}, w\right)=w \quad \text { pour }\left(z_{n+1}, w\right) \in \Delta_{n+1} \times D^{*}((0,0)) .
\end{aligned}
$$

Par (9.11) et la construction de $\psi$, il suffit pour notre but ( $\mathscr{E})$ de vérifier

$$
(\mathscr{E} *) \quad \frac{\partial \psi}{\partial \bar{z}_{i}}\left(0, z_{n+1}, w\right)=0 \quad(i=1, \cdots, n) \quad \operatorname{pour}\left(z_{n+1}, w\right) \in \Delta_{n+1} \times D^{*}((0,0)) .
$$

On regarde $z_{n+1}$ comme paramètre complexe dans $\Delta_{n+1}$. On considère deux restrictions du triple $\left(D^{*}, \Delta \times \Delta_{n+1}, p^{*}\right)$ sur $\Delta \times\{0\}$ et $\Delta \times\left\{z_{n+1}\right\}$ qu'on écrit $\left(D^{*}\left|0, \Delta, p^{*}\right| 0\right)$ et $\left(D^{*}\left|z_{n+1}, \Delta, p^{*}\right| z_{n+1}\right)$ respectivement. On prend $\left(D^{*} \mid 0, \Delta\right.$, $\left.p^{*} \mid 0\right) ; \quad\left(D^{*}\left|z_{n+1}, \Delta, p^{*}\right| z_{n+1}\right) ; \quad \xi^{*}(z, 0 ; \zeta) ; \quad \zeta^{*}\left(z, z_{n+1} ; \zeta\right) ; \psi\left(z, z_{n+1}, w\right)$ comme $(D, \Delta, p) ;(\tilde{D}, \Delta, \tilde{p}) ; \xi(z, \zeta) ; \eta(z, \zeta) ; \varphi(z, w)$ de p. 54, respectivement. Les conditions ci-dessus $\left(D^{*} 0\right) ;\left(D^{*} 1\right) ;\left(D^{*} 2\right) ;(9.9)$ entraînent respectivement celles $\left(E^{\prime} 0\right)$; $\left(E^{\prime} 1\right)$; $\left(E^{\prime} 2\right) ;\left(E^{\prime} 3\right)$ pour cette substitution. Il s'ensuit que l'inégalité (9.8) pour $i=1, \cdots, n$ est applicable, d'où

$$
\begin{gathered}
\iint_{D^{*}((0,0))}\left|\frac{\partial}{\partial w}\left(\frac{\partial \psi}{\partial \bar{z}_{i}}\left(z, z_{n+1}, w\right) \frac{\partial u(0,0, w)}{\partial w}\right)\right|_{z=0}^{2} d u d v \\
\leqq \operatorname{Im} \int_{\partial D^{*}((0,0))}\left\{\frac { \partial u ( 0 , 0 , w ) } { \partial w } \left(\frac{\partial \xi^{*}\left(z, z_{n+1} ; w\right)}{\partial z_{i}}\right.\right. \\
\left.\left.-\frac{\partial \xi^{*}(z, 0 ; w)}{\partial z_{i}}\right) \frac{\partial^{2} u(z, 0, w)}{\partial \bar{z}_{i} \partial w}\right\}_{z=0} d w
\end{gathered}
$$

Puisque le second membre ne dépend de que $z_{n+1}$ dans $\Delta_{n+1}$, on l'écrit $H\left(z_{n+1}\right)$. 
Il est évident que $H(0)=0$, et par l'analycité de $\xi^{*}\left(z, z_{n+1} ; \zeta\right)$ pour $z_{n+1} \in \Delta_{n+1}$, $H\left(z_{n+1}\right)$ est fonction harmonique réelle dans $\Delta_{n+1}$. En autre côté, d'après l'inégalité ci-dessus, $H\left(z_{n+1}\right) \geqq 0$ pour tout $z_{n+1} \in \Delta_{n+1}$. D'après le principe de maximum, on a $H\left(z_{n+1}\right)=0$ dans $\Delta_{n+1}$, d'où

$$
\left\{\frac{\partial}{\partial w}\left(\frac{\partial \psi}{\partial \bar{z}_{i}}\left(z, z_{n+1}, w\right) \frac{\partial u(0,0, w)}{\partial w}\right)\right\}_{z=0}=0 \quad \operatorname{pour}\left(z_{n+1}, w\right) \in \Delta_{n+1} \times D^{*}((0,0)) .
$$

Puisqu'il existe, d'après (9.9), au moins deux telles fonctions non-constantes $u(0,0, w)$ dans $D^{*}((0,0))$ linéairement indépendantes, on obtient $\left(\partial \psi / \partial \bar{z}_{i}\right)$. $\left(0, z_{n+1}, w\right)=0$ dans $\Delta_{n+1} \times D^{*}((0,0))$, d'où $\left(\mathscr{E}^{*}\right)$. Le théorème est complètement demontré.

\section{Références}

[1] L. V. Ahlfors and L. Sario, Riemann surfnces, Princeton Univ. Press, N. J., 1960.

[2] H. Behnke und P. Thullen, Theorie der Funktionen mehrerer komplexer veränderlichen, Chelsea, New York, 1934.

[3] S. Bergmann, The kernel function and conformal mapping, Amer. Math. Soc., 1950.

[4] J. Hadamard, Oeuvres de J. Hadamard, C.N.R.S., Paris, 1968.

[5] Y. Kusunoki, On generalized Dirichlet problem for plurisubharmonic functions, J. Math. Kyoto Univ., 4 (1964), 123-147.

[6] E. E. Levi, Studii sui punti singolari essenziali delle funzioni analitiche di due o più variabili complesse, Ann. di Mat., 17 (1909), 61-87.

[ 7 ] P. Lévy, Les équations intégro-différentielles définissant des fonctions de lignes, Thèse, $1911,120 \mathrm{pp}$.

[ 8 ] P. Lévy, Leçons d'analyse fonctionnelle, Gauthier-Villars, Paris, 1922 .

[9] T. Nishino, Sur la distribution des valeurs des fonctions analytiques de deux variables complexes (japonais), Sûgaku, 32 (1980), 230-246.

[10] K. Oka, Sur les fonctions analytiques de plusieurs variables, Iwanami Shoten, Tokyo, 1961.

[11] M. Schiffer, The kernel function of an orthogonal system, Duke Math. J., 13 (1946), 529-540.

[12] N. Suita, Capacities and kernels on Riemann surfaces, Arch. Rational Mech. Anal., 46 (1972), 212-217.

[13] H. Yamaguchi, Sur le mouvement des constantes de Robin, J. Math. Kyoto Univ., 15 (1975), 53-71.

[14] H. Yamaguchi, Parabolicité d'une fonction entière, J. Math. Kyoto Univ., 16 (1976), 71-92.

[15] H. Yamaguchi, Famille holomorphe de surfaces de Riemann ouvertes, qui est une variété de Stein, J. Math. Kyoto Univ., 16 (1976), 497-530.

[16] H. Yamaguchi, Variations de surfaces de Riemann, C. R. Acad. Sci. Paris, 286 (1978), 1121-1124.

[17] H. Yamaguchi, Forme de Levi et l'équation $\partial^{2} u / \partial z \partial \bar{z}=e^{2 u}$, C. R. Acad. Sci. Paris, 288 (1979), 619-622. 